\title{
AFFINE PROCESSES ON POSITIVE SEMIDEFINITE MATRICES
}

\author{
CHRISTA CUCHIERO, DAMIR FILIPOVIĆ, EBERHARD MAYERHOFER, AND \\ JOSEF TEICHMANN
}

\begin{abstract}
This paper provides the mathematical foundation for stochastically continuous affine processes on the cone of positive semidefinite symmetric matrices. These matrix-valued affine processes have arisen from a large and growing range of useful applications in finance, including multi-asset option pricing with stochastic volatility and correlation structures, and fixed-income models with stochastically correlated risk factors and default intensities.
\end{abstract}

\section{Contents}

1. Introduction

1.1. Program of the Article

1.2. Notation

2. Definition and Characterization of Affine Processes

2.1. Discussion of the Parameters

3. Affine Processes are Regular and Feller

4. Necessary Parameter Restrictions

4.1. The Functions $\mathrm{F}$ and $\mathrm{R}$

4.2. Infinitesimal Generator

4.3. Linear Transformations and Canonical Representation

4.4. Condition on the Constant Drift

5. Sufficient Conditions for the Existence and Uniqueness of Affine

\section{Processes}

5.1. Generalized Riccati Differential Equations

5.2. The Martingale Problem for $\mathcal{A}$

5.3. An Alternative Existence Proof for Jump Processes

6. Proof of the Main Results

6.1. Proof of Theorem 2.4

6.2. Proof of Theorem 2.5

6.3. Proof of Theorem 2.7

6.4. Proof of Theorem 2.10

Appendix A. Existence and Viability of a Class of Jump-diffusions

A.1. Diffusion Stochastic Differential Equations

Date: 30 September 2009.

Key words and phrases. affine process, stochastic invariance, stochastic volatility. MSC 2000: Primary: 60J25; Secondary: 91B70.

We thank Martin Keller-Ressel and Alexander Smirnov for discussions and helpful comments. Authors Christa Cuchiero and Josef Teichmann gratefully acknowledge the support from the FWF-grant Y328 (START prize from the Austrian Science Fund). Authors Damir Filipović and Eberhard Mayerhofer gratefully acknowledge the support from WWTF (Vienna Science and Technology Fund). 
A.2. Existence of Jump-diffusions 53

A.3. Viability of Jump-diffusions 54

Appendix B. An Approximation Lemma on the Cone of Positive

Semidefinite Matrices

References

\section{INTRODUCTION}

This paper provides the mathematical foundation for stochastically continuous affine processes on the cone of positive semidefinite symmetric $d \times d$-matrices $S_{d}^{+}$. These matrix-valued affine processes have arisen from a large and growing range of useful applications in finance, including multi-asset option pricing with stochastic volatility and correlation structures, and fixed-income models with stochastically correlated risk factors and default intensities.

For illustration, let us consider a multi-variate stochastic volatility model consisting of a $d$-dimensional logarithmic price process with risk-neutral dynamics

$$
d Y_{t}=\left(r \mathbf{1}-\frac{1}{2} X_{t}^{\mathrm{diag}}\right) d t+\sqrt{X_{t}} d B_{t}, \quad Y_{0}=y,
$$

and stochastic covariation process $X=\langle Y, Y\rangle$, which is a proxy for the instantaneous covariance of the price returns. Here $B$ denotes a standard $d$-dimensional Brownian motion, $r$ the constant interest rate, 1 the vector whose entries are all equal to one and $X^{\text {diag }}$ the vector containing the diagonal entries of $X$.

The necessity to specify $X$ as a process in $S_{d}^{+}$to qualify as covariation process is one of the mathematically interesting and demanding aspects of such models. Beyond that, the modeling of $X$ must allow for enough flexibility in order to reflect the stylized facts of financial data and to adequately capture the dependence structure of the different assets. If these requirements are met, the model can be used as a basis for financial decision-making in the area of portfolio optimization, pricing of multi-asset options and hedging of correlation risk.

The tractability of such a model crucially depends on the dynamics of $X$. A large part of the literature in the area of multivariate stochastic volatility modeling has proposed the following affine dynamics for $X$

$$
\begin{aligned}
d X_{t} & =\left(b+H X_{t}+X_{t} H^{\top}\right) d t+\sqrt{X_{t}} d W_{t} \Sigma+\Sigma^{\top} d W_{t}^{\top} \sqrt{X_{t}}+d J_{t}, \\
X_{0} & =x \in S_{d}^{+},
\end{aligned}
$$

where $b$ is some suitably chosen matrix in $S_{d}^{+}, H, \Sigma$ some invertible matrices, $W$ a standard $d \times d$-matrix of Brownian motions possibly correlated with $B$, and $J$ a pure jump process whose compensator is an affine function of $X \mathbb{1}$

The main reason for the analytic tractability of this model is that, under some technical conditions, the following affine transform formula holds:

$$
\mathbb{E}\left[e^{-\operatorname{Tr}\left(z X_{t}\right)+v^{\top} Y_{t}}\right]=e^{\Phi(t, z, v)+\operatorname{Tr}(\Psi(t, z, v) x)+v^{\top} y}
$$

\footnotetext{
${ }^{1}$ This affine multi-variate stochastic volatility model generalizes the well-known onedimensional models of Heston [28, for the diffusion case, or the Barndorff-Nielsen Shepard model [3], for the pure jump case.
} 
for the appropriate arguments $z \in S_{d} \times \mathrm{i} S_{d}$ and $v \in \mathbb{C}^{d}$. The functions $\Phi$ and $\Psi$ solve a system of non-linear ordinary differential equations (ODEs), which are determined by the model parameters. Setting $v=0, \phi(t, z)=-\Phi(t, z, 0)$ and $\psi(t, z)=-\Psi(t, z, 0)$ and taking $z=u \in S_{d}^{+}$, we arrive at

$$
\mathbb{E}\left[e^{-\operatorname{Tr}\left(u X_{t}\right)}\right]=e^{-\phi(t, u)-\operatorname{Tr}(\psi(t, u) x)}, \quad u \in S_{d}^{+} .
$$

In this paper, we characterize the class of all stochastically continuous timehomogeneous Markov processes - henceforth called affine processes - on $S_{d}^{+}$with the key property (1.3). Our main result shows that an affine process is necessarily a Feller process whose generator has affine coefficients in the state variables. The parameters of the generator satisfy some well-determined admissibility conditions, and are in a one-to-one relation with those of the corresponding ODEs for $\phi$ and $\psi$. Conversely, and more importantly for applications, we show that for any admissible parameter set there exists a unique well-behaved affine process on $S_{d}^{+}$. Furthermore, we prove that any stochastically continuous infinitely decomposable Markov process on $S_{d}^{+}$is affine with zero diffusion, and vice versa.

On the one hand, our findings extend the model class (1.2) since a more general drift and jumps are possible. Indeed, we allow for full generality in $b$ as long as $b-(d-1) \Sigma^{T} \Sigma \in S_{d}^{+}$, a general linear drift part $B(x)=\sum_{i j} x_{i j} \beta^{i j}$ and an inclusion of (infinite activity) jumps. This of course enables more flexibility in financial modeling. For example, due to the general linear drift part, the volatility of one asset can generally depend on the other ones which is not possible for $B(x)=$ $H x+x H^{\top}$. On the other hand, we now know the exact assumptions under which affine processes on $S_{d}^{+}$actually exist. Our characterization of affine processes on $S_{d}^{+}$ is thus exhaustive. Beyond that, the equivalence of infinitely decomposable Markov processes with state space $S_{d}^{+}$and affine processes without diffusion is interesting in its own right.

This paper complements Duffie et al. 17, who analyzed time-homogeneous affine processes on the state space $\mathbb{R}_{+}^{m} \times \mathbb{R}^{n} 2$ Matrix-valued affine processes seem to have been studied systematically for the first time in the literature by Bru 6, 7, who introduced the so called Wishart processes. These are generalizations of squares of matrix Ornstein-Uhlenbeck processes, that is, of the form (1.2) for $J=0$ and $b=k \Sigma^{\top} \Sigma$, for some real parameter $k>d-1$. Note that $k>d-1$ is a stronger assumption than what we require on $b$ and $\Sigma^{\top} \Sigma$. Bru [7] then establishes existence and uniqueness of a loca $3 S_{d}^{+}$-valued solution to (1.2) under the additional assumptions that $X_{0}$ has distinct eigenvalues, $-H \in S_{d}^{+}$, and that $H$ and $\Sigma$ commute (see [7. Theorem 2"]). In the more special case where $H=0$, Bru [7] shows global existence and uniqueness for (1.2) for any $X_{0} \in S_{d}^{+}$(see [7. Theorem 2 and last part of Section 3]) 4 Wishart processes have subsequently been introduced in the financial literature by Gourieroux and Sufana 25, 26] and Gourieroux et al. 24. Financial applications thereof have then been taken up and carried further by various authors,

\footnotetext{
${ }^{2}$ For the diffusion case see also 21] or [20, Chapter 10]. Time-inhomogeneous affine processes on $\mathbb{R}_{+}^{m} \times \mathbb{R}^{n}$ have been explored in [19].

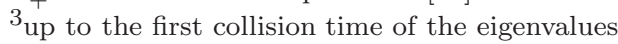

${ }^{4}$ Actually, Bru [7] establishes existence and uniqueness of solutions also for $k=1, \ldots, d-1$. But these are degenerate solutions, as they are only defined on low-dimensional subsets of the boundary of $S_{d}^{+}$(see [7] Corollary 1]).
} 
including Da Fonseca et al. [10, 11, 12, 13, and Buraschi et al. 99, 8. BarndorffNielsen and Stelzer [4 provide a theory for a certain class of matrix-valued Lévy driven Ornstein-Uhlenbeck processes of finite variation. Leippold and Trojani 39] introduce $S_{d}^{+}$-valued affine jump diffusions and provide financial examples, including multi-variate option pricing, fixed-income models and dynamic portfolio choice. All of these models are contained in our framework.

We want to point out that the full characterization of positive semidefinite matrix-valued affine processes needs a multitude of methods. In order to prove the fundamental property of regularity of affine processes another adaption of the famous analysis of Montgommery and Zippin is necessary, which has been worked out in [35] and [36] for the state space $\mathbb{R}_{+}^{m} \times \mathbb{R}^{n}$. For the necessary conditions on drift, diffusion and jump parameters we need the theory of infinitely divisible distributions on $S_{d}^{+}$. Most interestingly, the constant drift part $b$ must satisfy a condition depending on the magnitude of the diffusion component (see Proposition 4.17), which is in accordance with the choice of the drift in Bru's work [7] on Wishart processes, as we have seen above. This enigmatic additional condition on the drift $b$, is derived by refined methods from stochastic invariance theory. It is worth noting, as already visible in dimension one, that a naive application of classical geometric invariance conditions does not bring the correct necessary result on the drift but a stronger one. Indeed, take a one-dimensional affine diffusion process $X$ solving

$$
d X_{t}=b d t+\sqrt{X_{t}} d W_{t},
$$

then a back-of-the-envelope calculation would yield the Stratonovich drift at boundary point $x=0$ of value $b-\frac{1}{4}$ leading to the necessary parameter restriction $b \geq \frac{1}{4}$, which is too strong. It is well known that the correct parameter restriction is $b \geq 0$. The refined methods have to be introduced since precisely at the boundary of our state spaces the diffusion coefficients are not Lipschitz continuous anymore.

Refined stochastic invariance methods are also applied for the sufficient directions where existence - under the necessary parameter conditions - is shown through the solution of a martingale problem. Uniqueness follows by semigroup methods which need the theory of multi-dimensional Riccati equations.

To be precise we face two major problems in the analysis of positive matrix valued affine processes. First, the candidate stochastic differential equations necessarily lead to volatility terms which are not Lipschitz continuous at the boundary of the state space, which makes every existence, uniqueness and invariance question delicate. Second, the jump behavior near the boundary shows two distinguished features: parallel to the boundary diffusion-like behavior, i.e., infinite total variation can appear, whereas transversal to the boundary the process is only allowed to show finite total variation. This subtle situation leading to state dependent geometric conditions on the jump characteristics can be simplified through the affine property.

1.1. Program of the Article. For affine processes on $S_{d}^{+}$results and proofs deviate in essential points from the theory on state spaces of the form $\mathbb{R}_{+}^{m} \times \mathbb{R}^{n}$ given in [17, 35, which is a consequence of the more involved geometry of this nonpoyhedral cone. The program of the paper as outlined below therefore includes a comparison with the approach in [17.

Section 2 contains the main definition and a summary of the results of this article. In Section 3 we then derive two main properties, namely the regularity of the process and the Feller property of the associated semigroup. The Feller property, in 
turn, is a simple consequence of an important positivity result of the characteristic exponents $\phi, \psi$ which is proved in Lemma 3.3. This lemma is further employed as a tool for the treatment of the generalized Riccati differential equations in Section 5.1 (see the proof of Proposition 5.3). The global existence and uniqueness of these equations is then used to show uniqueness of the martingale problem for affine processes (see the proof of Proposition 5.9).

In Section 4, we define a set of admissible parameters specifying the infinitesimal generator of affine semigroups and prove the necessity of the parameter restrictions (see Proposition 4.9).

The sufficient direction is then treated in Section 5 . It is known that, for $d \geq 2$, there exist continuous affine processes on $S_{d}^{+}$which are - in contrast to those on the state space $\mathbb{R}_{+}^{m} \times \mathbb{R}^{n}$ - not infinitely divisible (see Example 2.9). The analysis of this paper reveals the failure of infinite divisibility as a consequence of the drift condition (see proof of Theorem 2.10). This has substantial influence on the approach chosen here to prove existence of affine processes associated with a given parameter set: Being in general hindered to recognize the solutions of the generalized Riccati differential equations as the logarithms of cumulant generating functions of substochastic measures, as done in [17, Section 6], we solve the martingale problem for the associated Lévy type generator on $S_{d}^{+}$, as exposed in Section 5 and Appendix A In Subsection 5.3. however, we deliver a variant of the existence proof of [17 for pure jump processes, which is possible in this case due to the absence of a diffusion component.

Finally, Section 6 contains the proofs of the main results which build on the results of the previous sections.

1.2. Notation. For the stochastic background and notation we refer to standard text books such as 32 and [43]. We write $\mathbb{R}_{+}=[0, \infty)$ and $\mathbb{R}_{++}=(0, \infty)$. Moreover:

- $S_{d}$ denotes the space of symmetric $d \times d$-matrices equipped with scalar product $\langle x, y\rangle=\operatorname{Tr}(x y)$. Note that $S_{d}$ is isomorphic, but not isometric, to the standard Euclidean space $\mathbb{R}^{d(d+1) / 2}$. We denote by $\left\{c^{i j}, i \leq j\right\}$ the standard basis of $S_{d}$, that is the $(k l)^{t h}$ component of $c^{i j}$ is given by $c_{k l}^{i j}=$ $\delta_{i k} \delta_{j l}+\delta_{j k} \delta_{i l}\left(1-\delta_{i j}\right)$, where $\delta_{i j}$ denotes the Kronecker delta. Additionally, we sometimes consider the following basis elements $\left\{e^{i j}, i \leq j\right\}$ which are positive semidefinite and form a basis of $S_{d}$

$$
e^{i j}= \begin{cases}c^{i i} & \text { if } i=j \\ c^{i i}+c^{i j}+c^{j j} & \text { if } i \neq j .\end{cases}
$$

- $S_{d}^{+}$stands for the cone of symmetric $d \times d$-positive semidefinite matrices, $S_{d}^{++}$for its interior in $S_{d}$, the cone of strictly positive definite matrices. The boundary is denoted by $\partial S_{d}^{+}=S_{d}^{+} \backslash S_{d}^{++}$, the complement is denoted by $\left(S_{d}^{+}\right)^{c}$, and $S_{d}^{+} \cup\{\Delta\}$ denotes the one-point compactification. Recall that $S_{d}^{+}$is self-dual (w.r.t. the scalar product $\langle x, y\rangle=\operatorname{Tr}(x y)$ ), that is,

$$
S_{d}^{+}=\left\{x \in S_{d} \mid\langle x, y\rangle \geq 0, \forall y \in S_{d}^{+}\right\} .
$$

Both cones, $S_{d}^{+}$and $S_{d}^{++}$, induce a partial and strict order relation on $S_{d}$, respectively: we write $x \preceq y$ if $y-x \in S_{d}^{+}$, and $x \prec y$ if $y-x \in S_{d}^{++}$.

- $M_{d}$ is the space of $d \times d$-matrices and $O(d)$ the orthogonal group of dimension $d$ over $\mathbb{R}$. 
- $I_{d}$ denotes the $d \times d$-identity matrix.

Throughout this paper, a function $f: S_{d} \rightarrow \mathbb{R}$ is understood as the restriction $f=\left.g\right|_{S_{d}}$ of a function $g: M_{d} \rightarrow \mathbb{R}$ which satisfies $g(x)=g\left(x^{\top}\right)$ for all $x \in M_{d}$. Without loss of generality $g(x)=f\left(\left(x+x^{\top}\right) / 2\right)$. We avoid using the vech operator, that is, to identify $x \in S_{d}$ with a vector in $\mathbb{R}^{d(d+1) / 2}$ by stringing the columns of $x$ together while only taking the entries $x_{i j}$ with $i \leq j$.

Throughout the article, we shall consider the following function spaces for measurable $U \subseteq S_{d}$. We write $\mathcal{B}(U)$ for the Borel $\sigma$-algebra on $U$. bU corresponds to the Banach space of bounded real-valued Borel measurable functions $f$ on $U$ with norm $\|f\|_{\infty}=\sup _{x \in U}|f(x)|$. We write $C(U)$ for the space of real-valued continuous functions $f$ on $U, C_{b}(U)$ for $C(U) \cap b U, C_{c}(U)$ for the space of functions $f \in C(U)$ with compact support and $C_{0}(U)$ for the Banach space of functions $f \in C(U)$ with $\lim _{x \rightarrow \Delta} f(x)=0$. Furthermore, $C^{k}(U)$ is the space of $k$ times differentiable functions $f$ on $U^{\circ}$, the interior of $U$, such that all partial derivatives of $f$ up to order $k$ belong to $C(U)$. As usual, we set $C^{\infty}(U)=\cap_{k \geq 1} C^{k}(U)$, and we write $C_{c}^{k}(U)=C_{c}(U) \cap C^{k}(U)$ and $C_{b}^{k}(U)=C_{b}(U) \cap C^{k}(U)$, for $k \leq \infty$.

\section{Definition and Characterization of Affine Processes}

We consider a time-homogeneous Markov process $X$ with state space $S_{d}^{+}$and semigroup $\left(P_{t}\right)_{t \geq 0}$ acting on functions $f \in b S_{d}^{+}$,

$$
P_{t} f(x)=\int_{S_{d}^{+}} f(\xi) p_{t}(x, d \xi), \quad x \in S_{d}^{+} .
$$

We note that $X$ may not be conservative. Then there is a standard extension of the transition probabilities to the one-point compactification $S_{d}^{+} \cup\{\Delta\}$ of $S_{d}^{+}$by defining

$$
p_{t}(x,\{\Delta\})=1-p_{t}\left(x, S_{d}^{+}\right), \quad p_{t}(\Delta,\{\Delta\})=1
$$

for all $t$ and $x \in S_{d}^{+}$, with the convention that $f(\Delta)=0$ for any function $f$ on $S_{d}^{+}$. Thus $X$ becomes conservative on $S_{d}^{+} \cup\{\Delta\}$.

Here is our main definition:

Definition 2.1. The Markov process $X$ is called affine if

(i) it is stochastically continuous, that is, $\lim _{s \rightarrow t} p_{s}(x, \cdot)=p_{t}(x, \cdot)$ weakly on $S_{d}^{+}$for every $t$ and $x \in S_{d}^{+}$, and

(ii) its Laplace transform has exponential-affine dependence on the initial state:

$$
P_{t} e^{-\langle u, x\rangle}=\int_{S_{d}^{+}} e^{-\langle u, \xi\rangle} p_{t}(x, d \xi)=e^{-\phi(t, u)-\langle\psi(t, u), x\rangle},
$$

for all $t$ and $u, x \in S_{d}^{+}$, for some functions $\phi: \mathbb{R}_{+} \times S_{d}^{+} \rightarrow \mathbb{R}_{+}$and $\psi: \mathbb{R}_{+} \times S_{d}^{+} \rightarrow S_{d}^{+}$.

Note that stochastic continuity of $X$ implies that $\phi(t, u)$ and $\psi(t, u)$ are jointly continuous in $(t, u)$, see Lemma 3.2(iii) below. In contrast to [17] we take stochastic continuity as part of the definition of affine processes, and consider the Laplace transform instead of the characteristic function. The latter is justified by the nonnegativity of $X$, the former is by convenience since, as we will see in Proposition 3.4 below, it automatically implies regularity in the following sense: 
Definition 2.2. The affine process $X$ is called regular if the derivatives

$$
F(u)=\left.\frac{\partial \phi(t, u)}{\partial t}\right|_{t=0+}, \quad R(u)=\left.\frac{\partial \psi(t, u)}{\partial t}\right|_{t=0+}
$$

exist and are continuous at $u=0$.

We remark that there are simple examples of Markov processes which satisfy Definition 2.1[(ii) but are not stochastically continuous, see [17, Remark 2.11]. However, such processes are of limited interest for applications and will not be considered.

In the following, we will provide an equivalent characterization of the affine property in terms of the generator of $X$. As we will see in (2.12), the diffusion, drift, jump and killing characteristics of $X$ depend in an affine way on the underlying state. The involved parameters are admissible in the following sense. Let $\chi$ : $S_{d} \rightarrow S_{d}$ denote some bounded continuous truncation function with $\chi(\xi)=\xi$ in a neighborhood of 0 .

Definition 2.3. An admissible parameter set $\left(\alpha, b, \beta^{i j}, c, \gamma, m, \mu\right)$ associated with $\chi$ consists of

- a linear diffusion coefficient

$$
\alpha \in S_{d}^{+}
$$

- a constant drift term

$$
b \succeq(d-1) \alpha,
$$

- a constant killing rate term

$$
c \in \mathbb{R}^{+},
$$

- a linear killing rate coefficient

$$
\gamma \in S_{d}^{+}
$$

- a constant jump term: a Borel measure $m$ on $S_{d}^{+} \backslash\{0\}$ satisfying

$$
\int_{S_{d}^{+} \backslash\{0\}}(\|\xi\| \wedge 1) m(d \xi)<\infty
$$

- a linear jump coefficient: a $d \times d$-matrix $\mu=\left(\mu_{i j}\right)$ of finite signed measures on $S_{d}^{+} \backslash\{0\}$ such that $\mu(E) \in S_{d}^{+}$for all $E \in \mathcal{B}\left(S_{d}^{+} \backslash\{0\}\right)$ and the kernel

$$
M(x, d \xi):=\frac{\langle x, \mu(d \xi)\rangle}{\|\xi\|^{2} \wedge 1}
$$

satisfies

$$
\int_{S_{d}^{+} \backslash\{0\}}\langle\chi(\xi), u\rangle M(x, d \xi)<\infty \quad \text { for all } x, u \in S_{d}^{+} \text {with }\langle x, u\rangle=0,
$$

- a linear drift coefficient: a family $\beta^{i j}=\beta^{j i} \in S_{d}$ such that the linear map $B: S_{d} \rightarrow S_{d}$ of the form

$$
B(x)=\sum_{i, j} \beta^{i j} x_{i j}
$$

satisfies

$$
\langle B(x), u\rangle-\int_{S_{d}^{+} \backslash\{0\}}\langle\chi(\xi), u\rangle M(x, d \xi) \geq 0 \quad \text { for all } x, u \in S_{d}^{+} \text {with }\langle x, u\rangle=0 \text {. }
$$


We will comment more on the admissibility conditions in Section 2.1 below. The following four theorems contain the main results of this article. Their proofs are given in Section [6. First, we provide a characterization of affine processes on $S_{d}^{+}$in terms of the admissible parameter set introduced in Definition 2.3. As for the domain of the generator, we consider the space $\mathcal{S}_{+}$of rapidly decreasing $C^{\infty}$. functions on $S_{d}^{+}$, defined in (B.1) below. It is shown in Section B that $e^{-\langle u, \cdot\rangle} \in \mathcal{S}_{+}$, for $u \in S_{d}^{++}$, as well as $C_{c}^{\infty}\left(S_{d}^{+}\right) \subset \mathcal{S}_{+}$.

Theorem 2.4. Suppose $X$ is an affine process on $S_{d}^{+}$. Then $X$ is regular and has the Feller property. Let $\mathcal{A}$ be its infinitesimal generator on $C_{0}\left(S_{d}^{+}\right)$. Then $\mathcal{S}_{+} \subset D(\mathcal{A})$ and there exists an admissible parameter set $\left(\alpha, b, \beta^{i j}, c, \gamma, m, \mu\right)$ such that, for $f \in \mathcal{S}_{+}$

$$
\begin{aligned}
\mathcal{A} f(x)= & \frac{1}{2} \sum_{i, j, k, l} A_{i j k l}(x) \frac{\partial^{2} f(x)}{\partial x_{i j} \partial x_{k l}}+\sum_{i, j}\left(b_{i j}+B_{i j}(x)\right) \frac{\partial f(x)}{\partial x_{i j}}-(c+\langle\gamma, x\rangle) f(x) \\
& +\int_{S_{d}^{+} \backslash\{0\}}(f(x+\xi)-f(x)) m(d \xi) \\
& +\int_{S_{d}^{+} \backslash\{0\}}(f(x+\xi)-f(x)-\langle\chi(\xi), \nabla f(x)\rangle) M(x, d \xi),
\end{aligned}
$$

where $B(x)$ is defined by (2.10), $M(x, d \xi)$ by (2.8) and

$$
A_{i j k l}(x)=x_{i k} \alpha_{j l}+x_{i l} \alpha_{j k}+x_{j k} \alpha_{i l}+x_{j l} \alpha_{i k} .
$$

Moreover, $\phi(t, u)$ and $\psi(t, u)$ in (2.1) solve the generalized Riccati differential equations, for $u \in S_{d}^{+}$,

$$
\begin{array}{ll}
\frac{\partial \phi(t, u)}{\partial t}=F(\psi(t, u)), & \phi(0, u)=0, \\
\frac{\partial \psi(t, u)}{\partial t}=R(\psi(t, u)), & \psi(0, u)=u
\end{array}
$$

with

$$
\begin{aligned}
& F(u)=\langle b, u\rangle+c-\int_{S_{d}^{+} \backslash\{0\}}\left(e^{-\langle u, \xi\rangle}-1\right) m(d \xi), \\
& R(u)=-2 u \alpha u+B^{\top}(u)+\gamma-\int_{S_{d}^{+} \backslash\{0\}}\left(\frac{e^{-\langle u, \xi\rangle}-1+\langle\chi(\xi), u\rangle}{\|\xi\|^{2} \wedge 1}\right) \mu(d \xi),
\end{aligned}
$$

where $B_{i j}^{\top}(u)=\left\langle\beta^{i j}, u\right\rangle$.

Conversely, let $\left(\alpha, b, \beta^{i j}, c, \gamma, m, \mu\right)$ be an admissible parameter set. Then there exists a unique affine process on $S_{d}^{+}$with infinitesimal generator (2.12) and (2.1) holds for all $(t, u) \in \mathbb{R}_{+} \times S_{d}^{+}$, where $\phi(t, u)$ and $\psi(t, u)$ are given by (2.14) and (2.15).

The affine transform formula (2.1) gives us the Laplace transform of the marginal distributions of $X$. From a computational point of view it is vital to know also the characteristic function of $X$. The following theorem asserts that.

Theorem 2.5. Suppose $X$ is an affine process on $S_{d}^{+}$. Then there exist a unique continuous $\left(\mathbb{R}_{+}+\mathrm{i} \mathbb{R}\right)$ - and $\left(S_{d}^{+}+\mathrm{i} S_{d}\right)$-valued extension of $\phi$ and $\psi$ to $\mathbb{R}_{+} \times\left(S_{d}^{+}+\mathrm{i} S_{d}\right)$, 
respectively, such that the affine transform formula (2.1) also holds for $u \in S_{d}^{+}+\mathrm{i} S_{d}$. Moreover, $\phi(\cdot, u)$ and $\psi(\cdot, u)$ solve (2.14) and (2.15) for all $u \in S_{d}^{+}+\mathrm{i} S_{d}$.

We remark that we could not find a direct proof of Theorem 2.5 other than by first establishing the real affine properties stated in Theorem 2.4 and then conclude by complex analytic continuation. See Section 6.2 below.

Remark 2.6. It can be proved as in [40] that $X$ is conservative if and only if $c=0$ and $\psi(t, 0) \equiv 0$ is the only $S_{d}^{+}$-valued local solution of (2.15) for $u=0$. The latter condition clearly requires that $\gamma=0$.

Hence, a sufficient condition for $X$ to be conservative is $c=0$ and $\gamma=0$ and

$$
\int_{S_{d}^{+} \cap\{\|\xi\| \geq 1\}}\|\xi\|\left(\mu_{i j}^{+}(d \xi)+\mu_{i j}^{-}(d \xi)\right)<\infty, \quad \text { for all } \quad 1 \leq i \leq j \leq d,
$$

where $\mu_{i j}=\mu_{i j}^{+}-\mu_{i j}^{-}$denotes the Jordan decomposition of $\mu_{i j}$. Indeed, it can be shown similarly as in [17. Section 9] that the latter property implies Lipschitz continuity of $R(u)$ on $S_{d}$.

Due to the Feller property, as established in Theorem 2.4 any affine process $X$ on $S_{d}^{+}$admits a càdlàg modification, still denoted by $X$ (see e.g. [43, Chapter III.2]). It can and will thus be realized on the space $\Omega=\mathbb{D}\left(S_{d}^{+} \cup\{\Delta\}\right)$ of càdlàg paths $\omega: \mathbb{R}_{+} \rightarrow S_{d}^{+} \cup\{\Delta\}$ with $\omega(s)=\Delta$ for $s>t$ whenever $\omega(t-)=\Delta$ or $\omega(t)=\Delta$. For every $x \in S_{d}^{+}$, we denote by $\mathbb{P}_{x}$ the law of $X$ given $X_{0}=x$ and by $\left(\mathcal{F}_{t}^{X}\right)$ the natural filtration generated by $X_{t}$. We also consider the usual augmentation

$$
\widetilde{\mathcal{F}}_{t}:=\bigcap_{x \in S_{d}^{+}} \mathcal{F}_{t}^{(x)}
$$

of $\left(\mathcal{F}_{t}^{X}\right)$, where $\left(\mathcal{F}_{t}^{(x)}\right)$ is the augmentation of $\left(\mathcal{F}_{t}^{X}\right)$ with respect to $\mathbb{P}_{x}$. Then $\left(\widetilde{\mathcal{F}}_{t}\right)$ is right continuous and $X$ is still a Markov process under $\left(\widetilde{\mathcal{F}}_{t}\right)$. We shall now relate conservative affine processes to semimartingales, where semimartingales are understood with respect to the stochastic basis $\left(\Omega, \widetilde{\mathcal{F}},(\widetilde{\mathcal{F}})_{t}, \mathbb{P}_{x}\right)$ for every $x$.

Theorem 2.7. Let $X$ be a conservative affine process on $S_{d}^{+}$and let $\left(\alpha, b, \beta^{i j}, c=\right.$ $0, \gamma=0, m, \mu)$ be the related admissible parameter set associated with the truncation function $\chi$. Then $X$ is a semimartingale whose characteristics $(B, A, \nu)$ with respect to $\chi$ are given by

$$
\begin{aligned}
A_{t, i j k l} & =\int_{0}^{t} A_{i j k l}\left(X_{s}\right) d s, \\
B_{t} & =\int_{0}^{t}\left(b+\int_{S_{d}^{+} \backslash\{0\}} \chi(\xi) m(d \xi)+B\left(X_{s}\right)\right) d s, \\
\nu(d t, d \xi) & =\left(m(d \xi)+M\left(X_{t}, d \xi\right)\right) d t,
\end{aligned}
$$

where $B(x)$ is given by (2.10), $A_{i j k l}(x)$ by (2.13) and $M(x, d \xi)$ by (2.8). Furthermore, there exists, possibly on an enlargement of the probability space, a $d \times d$-matrix 
of standard Brownian motions $W$ such that $X$ admits the following representation

$$
\begin{aligned}
X_{t}= & x+B_{t}+\int_{0}^{t}\left(\sqrt{X_{s}} d W_{t} \Sigma+\Sigma^{\top} d W_{s} \sqrt{X_{s}}\right) d s \\
& +\int_{0}^{t} \int_{S_{d}^{+} \backslash\{0\}} \chi(\xi)\left(\mu^{X}(d t, d \xi)-\nu(d t, d \xi)\right) \\
& +\int_{0}^{t} \int_{S_{d}^{+} \backslash\{0\}}(\xi-\chi(\xi)) \mu^{X}(d t, d \xi),
\end{aligned}
$$

where $\Sigma \in M_{d}$ satisfies $\Sigma^{\top} \Sigma=\alpha$ and $\mu^{X}$ denotes the random measure associated with the jumps of $X$.

Hence $X$ is continuous if and only if $m$ and $\mu$ vanish.

Let $\mathcal{P}$ be the set of all families of probability measures $\left(\mathbb{P}_{x}\right)_{x \in S_{d}^{+}}$on the canonical probability space $\left(\Omega, \mathcal{F}^{X}\right)$ such that $\left(X,\left(\mathbb{P}_{x}\right)_{x \in S_{d}^{+}}\right)$is a stochastically continuous Markov processes on $S_{d}^{+}$with $\mathbb{P}_{x}\left[X_{0}=x\right]=1$, for all $x \in S_{d}^{+} 5$ For two probability measures $\mathbb{P}, \mathbb{Q}$ on $\left(\Omega, \mathcal{F}^{X}\right)$, the convolution $\mathbb{P} * \mathbb{Q}$ is defined as the image of $\mathbb{P} \times \mathbb{Q}$ on $\Omega \times \Omega$ under the map $\left(\omega, \omega^{\prime}\right) \mapsto \omega+\omega^{\prime}$.

Definition 2.8. An element $\left(\mathbb{P}_{x}\right)_{x \in S_{d}^{+}} \in \mathcal{P}$ is called

(i) infinitely decomposable, if for each $k \geq 1$, there exists $\left(\mathbb{P}_{x}^{(k)}\right)_{x \in S_{d}^{+}} \in \mathcal{P}$ such that

$$
\mathbb{P}_{x^{(1)}+\cdots+x^{(k)}}=\mathbb{P}_{x^{(1)}}^{(k)} * \cdots * \mathbb{P}_{x^{(k)}}^{(k)}
$$

(ii) infinitely divisible, if the one-dimensional marginal distributions $\mathbb{P}_{x} \circ X_{t}^{-1}$ are infinitely divisible, for all $(t, x) \in S_{d}^{+}$.

In [17] it was shown that regular affine processes on $\mathbb{R}_{+}^{m} \times \mathbb{R}^{n}$ are infinitely decomposable Markov processes, and vice versa. In fact, this property was the core for the existence proof of affine processes in [17]. On $S_{d}^{+}$the situation is different, as the following counterexample reveals that not all affine processes on $S_{d}^{+}$are infinitely divisible:

Example 2.9. The affine process $X$ on $S_{d}^{+}$corresponding to the parameter set $\left(\alpha=I_{d}, b=\delta I_{d}, 0,0,0,0\right)$, where $\delta \in[d-1, \infty)$, is the diffusion process initially studied by Bru [7]. By [7, Theorem 3], the Laplace-transforms

$$
\mathbb{E}_{x}\left[e^{-\left\langle X_{t}, u\right\rangle}\right]=(\operatorname{det}(I+2 t u))^{\delta / 2} e^{-\left\langle(I+2 t u)^{-1} u, x\right\rangle}
$$

are those of non-central Wishart distributions WIS $(\delta, d, x)$. By a well known result due to Lévy, these Wishart distributions are not infinitely divisible if $d \geq 2$ (see Section 2.C in [16]).

Here is our main result on infinite divisibility of affine processes on $S_{d}^{+}$:

Theorem 2.10. Let $\left(\mathbb{P}_{x}\right)_{x \in S_{d}^{+}} \in \mathcal{P}$. The following are equivalent:

(i) $\left(\mathbb{P}_{x}\right)_{x \in S_{d}^{+}}$is infinitely decomposable.

(ii) $\left(X,\left(\mathbb{P}_{x}\right)_{x \in S_{d}^{+}}\right)$is affine with vanishing diffusion parameter $\alpha=0$.

(iii) $\left(X,\left(\mathbb{P}_{x}\right)_{x \in S_{d}^{+}}\right)$is affine and infinitely divisible.

\footnotetext{
${ }^{5}$ Note that in contrast to [17, there is no need to impose regularity of $X$.
} 
2.1. Discussion of the Parameters. We next discuss and highlight some properties of the admissible parameter set $\left(\alpha, b, \beta^{i j}, c, \gamma, m, \mu\right)$ of an affine process.

Let us therefore define the normal cone

$$
N_{S_{d}^{+}}(x)=\left\{u \in S_{d}^{+} \mid\langle u, x\rangle=0\right\},
$$

containing the inward pointing normal vectors, to $S_{d}^{+}$at $x \in S_{d}^{+} 6$ It will be shown in Lemma 4.1 below that $N_{S_{d}^{+}}(x) \neq\{0\}$ only for boundary elements $x \in \partial S_{d}^{+}$.

2.1.1. Diffusion. The diffusion term does not admit a constant part, and its linear part is of the very specific form

$$
\langle u, A(x) u\rangle=4\langle x, u \alpha u\rangle \mid
$$

We could thus write the second order differential operator in (2.12) as

$$
\frac{1}{2} \sum_{i, j, k, l} A_{i j k l}(x) \frac{\partial^{2} f(x)}{\partial x_{i j} \partial x_{k l}}=2\langle x, \nabla \alpha \nabla f(x)\rangle .
$$

The reason why we introduce and use the symmetrization (2.13) of $A(x)$ is that it corresponds to the quadratic characteristic (2.19) of the semimartingale $X$.

2.1.2. Drift. The remarkable drift condition (2.4) has been assumed in many previous papers. Here is the first time where necessity and sufficiency of (2.4) is proved in generality. Note that in dimension $d=1$, it simply reduces to non-negativity $b \geq 0$. But for dimension $d \geq 2$, the boundary of the state space $S_{d}^{+}$becomes curved, implying a non-trivial trade-off between diffusion $\alpha$ and $b$.

Concerning condition (2.11) and the form of $B$, let us note the following: Condition (2.11) implies in particular

$$
\beta_{\backslash\{i\}}^{i i}-\int_{S_{d}^{+} \backslash\{0\}} \frac{\chi(\xi) \backslash\{i\}}{\|\xi\|^{2} \wedge 1} \mu_{i i}(d \xi) \in S_{d-1}^{+} \text {for all } 1 \leq i \leq d,
$$

where for any matrix $u \in S_{d}, u_{\backslash\{i\}}$ denotes the matrix where the $i^{\text {th }}$ row and column are deleted. Indeed, inserting $x=c^{i i}$ in condition (2.11) yields

$$
\left\langle B\left(c^{i i}\right), u\right\rangle-\int_{S_{d}^{+} \backslash\{0\}} \frac{\langle\chi(\xi), u\rangle}{\|\xi\|^{2} \wedge 1} \mu_{i i}(d \xi) \geq 0
$$

for all $u \in S_{d}^{+}$with $\left\langle c^{i i}, u\right\rangle=0$. Since the $i^{\text {th }}$ column and row of such an element $u \in S_{d}^{+}$is zero, it follows that

$$
\begin{aligned}
& \left\langle B\left(c^{i i}\right), u\right\rangle-\int_{S_{d}^{+} \backslash\{0\}} \frac{\langle\chi(\xi), u\rangle}{\|\xi\|^{2} \wedge 1} \mu_{i i}(d \xi) \\
& =\left\langle\beta_{\backslash\{i\}}^{i i}, u \backslash\{i\}\right\rangle-\int_{S_{d}^{+} \backslash\{0\}} \frac{\left\langle\chi(\xi)_{\backslash\{i\}}, u \backslash\{i\}\right.}{\|\xi\|^{2} \wedge 1} \mu_{i i}(d \xi) \geq 0 .
\end{aligned}
$$

\footnotetext{
${ }^{6}$ Indeed, we obtain 2.23 from the general definition in A.8 below by choosing $y=0$ and $y=2 x$, and using the self-duality of $S_{d}^{+}:\langle u, y\rangle \geq 0$ for all $y, u \in S_{d}^{+}$.

${ }^{7}$ This property of $A(x)$ has also been announced in [27, however, their proof seems to have some gaps.
} 
By choosing appropriate elements $u \backslash\{i\} \in S_{d-1}^{+}$we can further derive the integrability of $\chi(\xi)_{k l}$ for all $k \neq i, l \neq i$, which implies

$$
\int_{S_{d}^{+} \backslash\{0\}} \frac{\langle\chi(\xi) \backslash\{i\}, u \backslash\{i\}}{\|\xi\|^{2} \wedge 1} \mu_{i i}(d \xi)=\left\langle\int_{S_{d}^{+} \backslash\{0\}} \frac{\chi(\xi) \backslash\{i\}}{\|\xi\|^{2} \wedge 1} \mu_{i i}(d \xi), u \backslash\{i\}\right\rangle .
$$

As (2.25) and (2.26) must hold true for all $u_{\backslash\{i\}} \in S_{d-1}^{+}$, assertion (2.24) is proved.

Note that the $(i j)^{t h}$ component of the adjoint operator $B^{\top}$ is given by

$$
B_{i j}^{\top}(u)=\left\langle\beta^{i j}, u\right\rangle
$$

since $\langle B(x), u\rangle=\left\langle\sum_{i, j} \beta^{i j} x_{i j}, u\right\rangle=\sum_{i, j}\left\langle\beta^{i j}, u\right\rangle x_{i j}=\left\langle B^{\top}(u), x\right\rangle$.

In most previous papers, $B(x)$ is of the form

$$
B(x)=H x+x H^{\top} .
$$

In this case

$$
\langle B(x), u\rangle=\left\langle H x+x H^{\top}, u\right\rangle=0 \quad \text { for all } x, u \in S_{d}^{+} \text {with }\langle x, u\rangle=0,
$$

and hence (2.11) is equivalent to

$$
\int_{S_{d}^{+} \backslash\{0\}}\langle\chi(\xi), u\rangle M(x, d \xi)=0
$$

for all $x, u \in S_{d}^{+}$with $\langle x, u\rangle=0$.

If $B(x)$ is of the form

$$
B(x)=H x+x H^{\top}+\Gamma(x)
$$

where $H \in M_{d}$ and $\Gamma: S_{d} \rightarrow S_{d}$ linear satisfying $\Gamma\left(S_{d}^{+}\right) \subseteq S_{d}^{+}$, then, in view of (2.29), condition (2.11) holds true as long as

$$
\langle\Gamma(x), u\rangle-\int_{S_{d}^{+} \backslash\{0\}}\langle\chi(\xi), u\rangle M(x, d \xi) \geq 0
$$

for all $x, u \in S_{d}^{+}$with $\langle x, u\rangle=0$. As a bold conjecture, we claim that any $B(x)$ satisfying (2.11) is of form (2.30).

Here is a simple example where $B(x)$ is of the form (2.30) but not of the usual form (2.28): let $d=2$ and $B(x)=\left(\begin{array}{ll}x_{22} & x_{12} \\ x_{12} & x_{11}\end{array}\right)$. It can be easily checked that (2.11) is satisfied, while $B(x)$ cannot be brought into the form (2.28). If $x_{i i}$ models the (squared) volatility of the $i$ th stock price, as in (1.1), then this drift specification admits level impacts of the volatility of stock 1 on the volatility of stock 2 , and vice versa.

\subsubsection{Killing. See Remark 2.6}

2.1.4. Jumps. Condition (2.7) means that jumps described by $m$, which can for instance appear at $x=0$ should be of finite variation entering the cone $S_{d}^{+}$, since infinite-variation transversal to the boundary would let the process leave the state space. Similarly, condition (2.9) asserts finite variation for the inward pointing direction, while we can have a general jump behavior (supported by $S_{d}^{+}$due to the affine structure) parallel to the boundary.

An example of a such a jump measure in dimension $d \geq 2$ can be constructed as follows: Let $g^{O}: S_{d}^{+} \rightarrow S_{d}^{+}, x \mapsto O^{\top} x O$ for an orthogonal matrix $O \in O(d)$. 
We consider now a kernel $M(x, d \xi)$ satisfying the admissibility conditions (2.8) and (2.9), with the additional property that

$$
M(x, d \xi)=g_{*}^{O} M\left(O x O^{\top}, d \xi\right) \quad \text { for all } O \in O(d),
$$

where $g_{*}^{O} M$ is the pushforward of $M$ with respect to $g^{O}$. This is equivalent to the requirement that the associated matrix of signed measures $\mu$ satisfies

$$
\mu(d \xi)=O^{\top} g_{*}^{O} \mu(d \xi) O \quad \text { for all } O \in O(d) .
$$

Such measures can be constructed by averaging over $O(d)$ with respect to the Haar measure (see e.g. 38, Chapter XII]). Moreover, note that by Proposition 4.12 below, every orthogonal transformation of an affine process with linear jump measure $\mu$ satisfying (2.32) has again linear jump measure $\mu$.

In order to define some kernel $M(x, d \xi)$ which allows for general jump behavior parallel to the boundary and guarantees finite variation for the inward pointing direction, it suffices - due to (2.31) - to specify $M(x, d \xi)$ for $x=c^{11}$ or equivalently to specify $\mu_{11}$. For example, a measure $\mu_{11}$ defined by

$$
\mu_{11}=\frac{\|\xi\|^{2} \wedge 1}{\xi_{11}^{2}+\sum_{i>1} \xi_{i i}} \nu(d \xi)
$$

where $\nu$ is some appropriately chosen finite positive measure, does the job. The condition on $\nu$ is the following,

$$
\int_{\|\xi\| \leq 1} \frac{\xi_{11}}{\xi_{11}^{2}+\sum_{i>1} \xi_{i i}} \nu(d \xi)=\infty
$$

meaning that $M(x, d \xi)$ does not admit finite total variation parallel to the boundary. All other conditions on $\mu_{11}$ from (2.24) are fullfilled, since

$$
\int_{\|\xi\| \leq 1} \frac{\xi_{i j}}{\xi_{11}^{2}+\sum_{i>1} \xi_{i i}} \nu(d \xi)
$$

is finite for $i, j>1$.

The linear drift part $B$, for which we require

$$
\left\langle B^{\top}(u), x\right\rangle=\left\langle B^{\top}\left(O u O^{\top}\right), O x O^{\top}\right\rangle,
$$

can then be specified similarly such that (2.9) is fulfilled. Indeed, it is sufficient to choose some $\beta^{11} \in S_{d}$ which satisfies property (2.24) for $i=1$, since by relation (2.33), $B$ is then already entirely determined and (2.9) holds true.

Therefore it is possible to construct affine processes with affine jump part without finite total variation. Such processes look parallel to the boundary "like" a diffusion but, for $d \geq 2$, one cannot approximate (in law) affine, positive matrix valued diffusion processes by them, since this would yield a contradiction to condition (2.4). See also Remark 5.11 below.

\section{Affine Processes are Regular and Feller}

Suppose $X$ is an affine process on $S_{d}^{+}$. The main result of this section is that $X$ is regular in the sense of Definition 2.1. In addition, we shall prove that $P_{t}$ is a Feller semigroup on $C_{0}\left(S_{d}^{+}\right)$. In order to show both properties, we shall mainly rely on Lemma 3.3 below. The Feller property is then a simple consequence of this statement and regularity is obtained by arguing as in Keller-Ressel, Schachermayer and Teichmann 36] who obtained the corresponding statements for affine processes on the state space $\mathbb{R}_{+}^{m} \times \mathbb{R}^{n}$, see [36, Theorem 4.3] and also the $\mathrm{PhD}$ thesis of 
Keller-Ressel [35. We observe that most arguments of [36] translate to our setting without major changes. It is only required to tailor some technicalities to the cone $S_{d}^{+}$. We start with the following elementary observations:

Lemma 3.1. If $u \in \partial S_{d}^{+}$and $S_{d}^{+} \ni v \preceq u$, then $v \in \partial S_{d}^{+}$.

Proof. Let $x \in S_{d}^{+} \backslash\{0\}$ such that $\langle x, u\rangle=0$. Then, $S_{d}^{+} \ni v \preceq u$ implies $0 \leq$ $\langle v, x\rangle \leq\langle u, x\rangle=0$. Hence $v \in \partial S_{d}^{+}$.

We now derive some first properties of the functions $\phi$ and $\psi$ in (2.1).

Lemma 3.2. Let $X$ be an affine process on $S_{d}^{+}$. Then, we have

(i) The functions $\phi$ and $\psi$ satisfy

$$
\begin{aligned}
& \phi(t+s, u)=\phi(t, u)+\phi(s, \psi(t, u)), \\
& \psi(t+s, u)=\psi(s, \psi(t, u)) .
\end{aligned}
$$

for all $t, s \in \mathbb{R}_{+}$.

(ii) For all $u, v \in S_{d}^{+}$with $v \preceq u$ and for all $t \geq 0$, the order relations

$$
\phi(t, v) \leq \phi(t, u) \quad \text { and } \quad \psi(t, v) \preceq \psi(t, u) .
$$

hold true.

(iii) The functions $\phi$ and $\psi$ are jointly continuous in $\mathbb{R}_{+} \times S_{d}^{+}$. Furthermore, $u \mapsto \phi(t, u)$ and $u \mapsto \psi(t, u)$ are analytic on $S_{d}^{++}$.

Proof. Assertion (i) follows directly from the Chapman-Kolmogorov equation,

$$
\begin{aligned}
e^{-\phi(t+s, u)-\langle\psi(t+s, u), x\rangle} & =\int_{S_{d}^{+}} p_{s}(x, d \xi) \int_{S_{d}^{+}} e^{-\langle u, \widetilde{\xi}\rangle} p_{t}(\xi, d \widetilde{\xi}) \\
& =e^{-\phi(t, u)} \int_{S_{d}^{+}} e^{-\langle\psi(t, u), \xi\rangle} p_{s}(x, d \xi) \\
& =e^{-\phi(t, u)-\phi(s, \psi(t, u))-\langle\psi(s, \psi(t, u)), x\rangle}
\end{aligned}
$$

For the proof of (ii) note that $v \preceq u$ is equivalent to $\langle v, x\rangle \leq\langle u, x\rangle$ for all $x \in S_{d}^{+}$. By the monotonicity of the exponential function, we have for all $x \in S_{d}^{+}$and for all $t \geq 0$,

$e^{-\phi(t, v)-\langle\psi(t, v), x\rangle}=\int_{S_{d}^{+}} e^{-\langle v, \xi\rangle} p_{t}(x, d \xi) \geq \int_{S_{d}^{+}} e^{-\langle u, \xi\rangle} p_{t}(x, d \xi)=e^{-\phi(t, u)-\langle\psi(t, u), x\rangle}$,

and the assertion follows by taking logarithms.

Concerning statement (iii) note that stochastic continuity of $X$ implies joint continuity of $P_{t} e^{-\langle u, x\rangle}$ in $(t, u) \in \mathbb{R}_{+} \times S_{d}^{+}$(this follows e.g. from [5] Lemma 23.7]), for all $x \in S_{d}^{+}$. This in turn yields continuity of the functions $(t, u) \mapsto \phi(t, u)$ and $(t, u) \mapsto \psi(t, u)$. The second assertion follows from analyticity properties of the Laplace transform.

The following property of $\psi$ is crucial.

Lemma 3.3. Let $\psi: \mathbb{R}_{+} \times S_{d}^{+} \rightarrow S_{d}^{+}$be any map satisfying properties (i) (iii) of Lemma 3.2. Then $\psi(t, u) \in S_{d}^{++}$for all $u \in S_{d}^{++}$and $t \geq 0$. 
Proof. We adapt the proof of [35. Proposition 1.10] to our setting. Assume by contradiction that there exists some $(t, u) \in \mathbb{R}_{+} \times S_{d}^{++}$such that $\psi(t, u) \in \partial S_{d}^{+}$. Let us consider the interval $\left(0, \lambda_{\min }(u)\right] \neq \emptyset$, where $\lambda_{\min }(u)>0$ denotes the smallest eigenvalue of $u$. Then we have that $v I_{d} \preceq u$ for $v \in \mathbb{R}_{++}$is equivalent to $v \in$ $\left(0, \lambda_{\min }(u)\right]$. Since $\psi(t, u)$ admits property (ii) of Lemma 3.2 we obtain that

$$
S_{d}^{+} \ni \psi\left(t, v I_{d}\right) \preceq \psi(t, u) \in \partial S_{d}^{+}
$$

holds true for all $v \in\left(0, \lambda_{\min }(u)\right]$. Consequently, Lemma 3.1 yields that $\psi\left(t, v I_{d}\right) \in$ $\partial S_{d}^{+}$. Hence,

$$
\operatorname{det}\left(\psi\left(t, v I_{d}\right)\right)=0
$$

for all $v \in\left(0, \lambda_{\min }(u)\right]$. The analyticity of $u \mapsto \psi(t, u)$ on $S_{d}^{++}$carries over to $u \mapsto \operatorname{det}(\psi(t, u))$ and implies that $\operatorname{det}\left(\psi\left(t, v I_{d}\right)\right)=0$ for all $v \in \mathbb{R}_{++}$. Indeed, the set of zeros of $\operatorname{det}\left(\psi\left(t, v I_{d}\right)\right)$ has an accumulation point in $\mathbb{R}_{++}$, which implies that $\operatorname{det}\left(\psi\left(t, v I_{d}\right)\right)$ vanishes entirely on $\mathbb{R}_{++}$. The same statement holds true for $t$ replaced by $\frac{t}{2}$. Indeed, if $\psi\left(\frac{t}{2}, u\right) \in \partial S_{d}^{+}$, then the assertion is shown by the same arguments as above. Otherwise, if $\psi\left(\frac{t}{2}, u\right) \in S_{d}^{++}$, we have for all $v \in \mathbb{R}_{++}$with $v I_{d} \preceq \psi\left(\frac{t}{2}, u\right)$, that is for all $v \in\left(0, \lambda_{\min }\left(\psi\left(\frac{t}{2}, u\right)\right]\right.$

$$
S_{d}^{+} \ni \psi\left(\frac{t}{2}, v I_{d}\right) \preceq \psi\left(\frac{t}{2}, \psi\left(\frac{t}{2}, u\right)\right)=\psi(t, u) \in \partial S_{d}^{+},
$$

which yields again $\psi\left(\frac{t}{2}, v I_{d}\right) \in \partial S_{d}^{+}$and $\operatorname{det}\left(\psi\left(\frac{t}{2}, v I_{d}\right)\right)=0$ for all $v \in\left(0, \lambda_{\min }\left(\psi\left(\frac{t}{2}, u\right)\right]\right.$. The same reasoning as before then leads to $\operatorname{det}\left(\psi\left(\frac{t}{2}, v I_{d}\right)\right)=0$ for all $v \in \mathbb{R}_{++}$. By reapplying this argument we finally get for every $n \in \mathbb{N}$ and for all $v \in \mathbb{R}_{++}$

$$
\operatorname{det}\left(\psi\left(\frac{t}{2^{n}}, v I_{d}\right)\right)=0
$$

From the continuity of the function $t \mapsto \psi(t, u)$ and of the determinant, we deduce that for any $v \in \mathbb{R}_{++}$,

$$
0=\lim _{n \rightarrow \infty} \operatorname{det}\left(\psi\left(\frac{t}{2^{n}}, v I_{d}\right)\right)=\operatorname{det}\left(\psi\left(0, v I_{d}\right)\right)=\operatorname{det}\left(v I_{d}\right)=v^{d}>0,
$$

a contradiction, and the assertion is proved.

We may now formulate the main result of this section:

Proposition 3.4. Let $X$ be an affine process with state space $S_{d}^{+}$. Then, we have:

(i) $X$ is a Feller process.

(ii) $X$ is regular.

Proof. In order to prove (i), it suffices to show that for all $f \in C_{0}\left(S_{d}^{+}\right)$

$$
\begin{gathered}
\lim _{t \rightarrow 0+} P_{t} f(x)=f(x), \quad \text { for all } x \in S_{d}^{+}, \\
P_{t} f \in C_{0}\left(S_{d}^{+}\right), \quad \text { for all } t \in \mathbb{R}_{+},
\end{gathered}
$$

(see for instance [43, Propostion III.2.4]). Property (3.4) is a consequence of stochastic continuity which implies for all $f \in C_{0}\left(S_{d}^{+}\right)$and $x \in S_{d}^{+}$

$$
\lim _{t \rightarrow 0^{+}} P_{t} f(x)=f(x) .
$$

Concerning (3.5), it suffices to verify this property for a dense subset of $C_{0}\left(S_{d}^{+}\right)$. By a locally compact version of Stone-Weierstrass' Theorem (see for example [48]), the linear span of the set $\left\{e^{-\langle u, x\rangle} \mid u \in S_{d}^{++}\right\}$is dense in $C_{0}\left(S_{d}^{+}\right)$. Indeed, it is 
a subalgebra of $C_{0}\left(S_{d}^{+}\right)$, separates points and vanishes nowhere, as all elements are strictly positive functions on $S_{d}^{+}$. From Proposition 3.3 we can deduce that $P_{t} e^{-\langle u, x\rangle} \in C_{0}\left(S_{d}^{+}\right)$if $u \in S_{d}^{++}$, whence statement (i) is proved.

The proof of (ii) follows precisely the lines of [36, Proof of Theorem 4.3]. Using Proposition 3.3 one may mimic the proof of [36. Theorem 4.3] to obtain that differentiability of $\psi(t, u)$ in $u \in S_{d}^{++}$, which follows from Lemma 3.2 (iii) implies differentiability of $\psi(t, u)$ in $t$ for $t=0$ and for all $u \in S_{d}^{+}$.

By the regularity of $X$ we are now allowed to differentiate the equations (3.1) and (3.2) with respect to $t$ and evaluate them at $t=0$. As a consequence, $\phi$ and $\psi$ satisfy the system of differential equations,

$$
\begin{aligned}
& \frac{\partial \phi(t, u)}{\partial t}=F(\psi(t, u)), \quad \phi(0, u)=0, \\
& \frac{\partial \psi(t, u)}{\partial t}=R(\psi(t, u)), \quad \psi(0, u)=u \in S_{d}^{+},
\end{aligned}
$$

where $F$ and $R$ are as defined in (2.2). The analysis of the these generalized Riccati differential equations is subject of Section 5.1, whereas the specific form of $F$ and $R$ is elaborated in the following.

\section{Necessary Parameter Restrictions}

In this section, we derive necessary parametric restrictions, that is, given an affine process on $S_{d}^{+}$, we determine necessary implications on a set of parameters which only ensue from Definition 2.1. These conditions are precisely the conditions on the admissible parameter set as of Definition 2.3. The form of the functions $F$ and $R$ as defined by (2.2) is then characterized by means of this parameter set, which is stated in Proposition 4.9 below. For its proof we first provide a number of technical prerequisites.

Lemma 4.1. Let $x, u \in S_{d}^{+}$and

$$
x=O \Lambda O^{\top}=O \operatorname{diag}\left(\lambda_{1}>0, \ldots, \lambda_{d-r}>0,0, \ldots, 0\right) O^{\top}
$$

be the diagonalization of $x$ with $r \geq 0$ and $O \in O(d)$. Then the following assertions are equivalent:

(i) $u x=x u=0$,

(ii) $\langle x, u\rangle=0$,

(iii) $u$ is of form

$$
u=O\left(\begin{array}{cc}
0 & 0 \\
0 & w
\end{array}\right) O^{\top}
$$

with $w \in S_{r}^{+}$.

Proof. The direction (i) $\Rightarrow$ (ii) is obvious. In order to prove the implication (ii) $\Rightarrow$ (iii) define $v$ as $v=O^{\prime} u O$. Then we have

$$
0=\langle x, u\rangle=\left\langle\Lambda, O^{\top} u O\right\rangle=\sum_{i \leq d-r} \lambda_{i} v_{i i},
$$

which implies $v_{i i}=0$ for all $i \leq d-r$ and by the positive definiteness of $v$ it must then be of form

$$
v=\left(\begin{array}{cc}
0 & 0 \\
0 & w
\end{array}\right)
$$


with $w \in S_{r}^{+}$. Thus $u$ is given by (4.2). This then implies that $u x=x u=0$, which proves the direction (iii) $\Rightarrow$ (i).

Lemma 4.2. Let $p$ be an orthogonal projector, that is, $p \in S_{d}^{+}$and $p^{2}=p$ (see e.g. Kato [34, Section I.6.7]), and define $q=I_{d}-p$. Then $q$ is an orthogonal projector and the orthogonal complement of $p$ in $S_{d}^{+}$equals

$$
\left\{v \in S_{d}^{+} \mid\langle p, v\rangle=0\right\}=\left\{q u q \mid u \in S_{d}^{+}\right\} .
$$

Proof. That $q$ is an orthogonal projector follows by inspection. The diagonalization of $p$ is of the form $p=O \Lambda O^{\top}$ with $\Lambda=\operatorname{diag}(1, \ldots, 1,0, \ldots, 0)$, and thus $q=$ $O\left(I_{d}-\Lambda\right) O^{\top}$. In view of Lemma 4.1 we conclude that $v \in S_{d}^{+}$is orthogonal to $p$ if and only if $v=q v q$. This proves the assertion.

Lemma 4.3. Let $u$ be in $S_{d}$ and $x \in \partial S_{d}^{+}$such that $u x=x u=0$. Then, the linear map $T_{u}$ defined by

$$
T_{u}: S_{d} \rightarrow S_{d}, \quad v \mapsto T_{u} v:=u v u
$$

has the following properties:

(i) $T_{u}$ is self-adjoint and $T_{u}\left(S_{d}^{+}-\mathbb{R}_{+} x\right) \subseteq S_{d}^{+}$.

(ii) There exists an element $v \in S_{d}$ such that $T_{u} v=u$

Proof. The assertion (i) is obvious, since for every $k \in \mathbb{R}_{+}, T_{u} k x=k u x u=0$ and $T_{u} v=u v u \in S_{d}^{+}$if $v \in S_{d}^{+}$. For proving part (ii) we use the fact that $x$ is of form (4.1) and that all zero divisors $u$ in $S_{d}$ of $x$ can be represented by (4.2) with $w \in S_{r}$. Thus, setting

$$
v=O\left(\begin{array}{cc}
0 & 0 \\
0 & w^{+}
\end{array}\right) O^{\top},
$$

where $w^{+}$satisfies $w w^{+} w=w$, yields $T_{u} v=u$.

Lemma 4.4. Let $V$ denote a vector spac 8 over $\mathbb{R}$. Let $L: S_{d}^{+} \rightarrow V$ be an additive (resp. homogeneous additive) map, that is, for all $x, y \in S_{d}^{+}$and $\lambda=1$ (resp. for all $\lambda \in \mathbb{R}_{+}$) we have,

$$
L(x+\lambda y)=L(x)+\lambda L(y) .
$$

Then $L(x)$ is the restriction of an additive (resp. $\mathbb{R}$-linear) map on $S_{d}$.

Proof. We define the map $\widetilde{L}: S_{d} \rightarrow V$ as

$$
\widetilde{L}(x-y):=L(x)-L(y), \quad x, y \in S_{d} .
$$

$\widetilde{L}$ is well defined, as for $u, v, w, z \in S_{d}^{+}$such that $u-v=w-z$ we have

$$
L(u)-L(v)=\widetilde{L}(u-v)=\widetilde{L}(w-z)=L(w)-L(z) .
$$

Since $S_{d}^{+}-S_{d}^{+}=S_{d}$, the domain of $\widetilde{L}$ is all of $S_{d}$. Also, $L(0)=0$ by the additivity of $L$. Hence $\widetilde{L}$ is the restriction of $L$ to $S_{d}^{+}$. Homogeneity of $\widetilde{L}$ holds, as for $\lambda>0, x=w-z \in S_{d}$ we have by definition

$$
\widetilde{L}(\lambda x)=L(\lambda w)-L(\lambda z)=\lambda L(w)-\lambda L(z)=\lambda \widetilde{L}(x) .
$$

Finally we show additivity of $\widetilde{L}$. Choose $x, y \in S_{d}$ such that $x=w-z, y=u-v$, hence $x+y=(w+u)-(z+v)$. By the definition of $\widetilde{L}$ we have

$$
\widetilde{L}(x)=L(w)-L(z), \quad \widetilde{L}(y)=L(u)-L(v),
$$

\footnotetext{
${ }^{8}$ In the proof of Proposition 4.9 below, $V$ corresponds to $S_{d}$, the vector space of linear maps $S_{d} \rightarrow S_{d}$, or the vector space of finite signed measures on $S_{d}$.
} 
and by the additivity of $L$ we obtain

$$
\widetilde{L}(x+y)=L(w+u)-L(z+v)=L(w)+L(u)-L(z)-L(v)=\widetilde{L}(x)+\widetilde{L}(y) .
$$

We provide the convergence result for Laplace transforms (in fact Laplace-Fourier transforms) which is most relevant for the analysis of affine processes.

Lemma 4.5. Let $\nu_{n}$ be a sequence of measures on $S_{d}$ with

$$
L_{n}(u)=\int_{S_{d}} e^{-\langle u, \xi\rangle} \nu_{n}(d \xi)<\infty \quad \text { and } \quad \lim _{n} L_{n}(u)=L(u), \quad u \in S_{d}^{+},
$$

pointwise, for some finite function $L$ on $S_{d}^{+}$continuous at $u=0$. Then $\nu_{n}$ converges weakly to some finite measure $\nu$ on $S_{d}$ and the Fourier-Laplace transforms converge for $u \in S_{d}^{++} \cup\{0\}$ and $v \in S_{d}$ to the Fourier-Laplace transforms of $\nu$, i.e.,

$$
\lim _{n} \int_{S_{d}} e^{-\langle u+i v, \xi\rangle} \nu_{n}(d \xi)=\int_{S_{d}} e^{-\langle u+i v, \xi\rangle} \nu(d \xi) .
$$

In particular, $\nu\left(S_{d}\right)=\lim _{n} \nu\left(S_{d}\right)$ and

$$
L(u)=\int_{S_{d}} e^{-\langle u, \xi\rangle} \nu(d \xi)
$$

for all $u \in S_{d}^{++} \cup\{0\}$.

Remark 4.6. Instead of $u=0$ we could take any set $K$ of points at the boundary $K \subset \partial S_{d}^{+}$: if we assume continuity of $L$ at points in $K$, then we obtain the equality of $L$ with the Laplace transform of $\nu$ for all points in $K$. Additionally continuity is too strong an assumption, since we only need right continuity of $L$ along the segment $u+\epsilon \operatorname{id}_{d}$ for $\epsilon=0$ at the points from the boundary under consideration.

Proof. Since $\nu_{n}\left(S_{d}\right)=L_{n}(0)$ is uniformly bounded in $n$, we know by general theory that $\nu_{n}$ has a vague accumulation point $\nu$, which is a finite measure on $S_{d}$.

Since $L_{n}(u)<\infty$ on $S_{d}^{+}$, it follows by well-known regularity properties of Laplace transforms (see e.g. [20, Lemma 10.8]) that the functions $L_{n}$ admit an analytic extension on the strip $S_{d}^{++}+i S_{d}$, still denoted by $L_{n}$ :

$$
(u+i v) \mapsto L_{n}(u+i v)=\int_{S_{d}} e^{-\langle u+i v, \xi\rangle} \nu_{n}(d \xi),
$$

and $\left|L_{n}(u+i v)\right| \leq L_{n}(u)$. Moreover, pointwise convergence of the finite convex functions $L_{n}$ to $L$ on $S_{d}^{+}$implies that this convergence is in fact uniform on compact subsets of $S_{d}^{++}$(see e.g. Rockafellar [44, Theorem 10.8]). Therefore, and since $S_{d}^{++}$ is a set of uniqueness in $S_{d}^{++}+i S_{d}$, it follows by Vitali's theorem (41, Chapter 1, Proposition 7]) that the analytic functions $L_{n}$ converge uniformly on compact subsets of $S_{d}^{++}+i S_{d}$ to an analytic limit thereon. Whence we know that for any $u \in S_{d}^{++}$the finite measures $\exp (-\langle u, \xi\rangle) \nu_{n}(d \xi)$ converge weakly to a limit, which by uniqueness of the weak limit has to equal $\exp (-\langle u, \xi\rangle) \nu(d \xi)$. Whence the only vague accumulation point of $\nu_{n}$ is $\nu$. Vague convergence implies weak convergence if mass is conserved. Continuity in turn of $L(u)$ at $u=0$ implies mass conservation: 
indeed by weak convergence of $e^{-\left\langle\epsilon I_{d}, \xi\right\rangle} \nu_{n}$ we arrive at

$$
\begin{aligned}
L\left(\epsilon I_{d}\right) & =\lim _{n} \int_{S_{d}} e^{-\left\langle\epsilon I_{d}, \xi\right\rangle} \nu_{n}(d \xi)=\int_{S_{d}} e^{-\left\langle\epsilon I_{d}, \xi\right\rangle} \nu(d \xi) \\
& =\int_{S_{d}} e^{-\left\langle\epsilon I_{d}, \xi\right\rangle} 1_{\left\{\left\langle I_{d}, \xi\right\rangle \leq 0\right\}} \nu(d \xi)+\int_{S_{d}} e^{-\left\langle\epsilon I_{d}, \xi\right\rangle} 1_{\left\{\left\langle I_{d}, \xi\right\rangle>0\right\}} \nu(d \xi)
\end{aligned}
$$

and therefore - by monotone convergence - we obtain that the limit $\epsilon \rightarrow 0$ yields

$$
L(0)=\int_{S_{d}} \nu(d \xi),
$$

which is the desired mass conservation, hence weak convergence, which means in turn convergence of the Fourier-Laplace transform at $u=0$.

Finally, let us state a general comparison result for ODEs and hereto introduce the notion of quasi-monotonicity, which we shall need several times throughout this article, in particular in the proofs of Propositions 4.9 and 5.3 below.

Definition 4.7. Let $U \subset S_{d}$ be an open set. A function $f: U \rightarrow S_{d}$ is called quasi-monotone increasing if for all elements $x, y \in U, u \in S_{d}^{+}$which satisfy $x \preceq y$ and $\langle x, u\rangle=\langle y, u\rangle$,

$$
\langle f(x), u\rangle \leq\langle f(y), u\rangle
$$

holds true. Accordingly, we call $f$ quasi-constant if both $f$ and $-f$ are quasimonotone increasing.

The following comparison result can be deduced from a more general theorem proved by Volkmann [51]:

Theorem 4.8. Let $U \subset S_{d}$ be an open set. Let $f:[0, T) \times U \rightarrow S_{d}$ be a continuous locally Lipschitz map such that $f(t, \cdot)$ is quasi-monotone increasing on $U$ for all $t \in[0, T)$. Let $0<t_{0} \leq T$ and $x, y:\left[0, t_{0}\right) \rightarrow U$ be differentiable maps such that $x(0) \preceq y(0)$ and

$$
\dot{x}(t)-f(t, x(t)) \preceq \dot{y}(t)-f(t, y(t)), \quad 0 \leq t<t_{0} .
$$

Then we have $x(t) \preceq y(t)$ for all $t \in\left[0, t_{0}\right)$.

4.1. The Functions $\mathbf{F}$ and $\mathbf{R}$. The main result of this section characterizes the form of the functions $F$ and $R$ as defined by (2.2).

Proposition 4.9. Let $X$ be an affine process with state space $S_{d}^{+}$. Then there exist parameters $\left(\alpha, b, \beta^{i j}, c, \gamma, m, \mu\right)$, where $\alpha, \beta^{i j}, c, \gamma, m, \mu$ satisfy the admissibility conditions of Definition 2.3 and $b \in S_{d}^{+}$, such that the functions $F$ and $R$ are of the form 2.16) and (2.17).

Remark 4.10. Note that for the moment we only obtain $b \in S_{d}^{+}$, and not (2.4).

Proof. As the proof of Proposition (4.9) is rather long we divide it into several steps:

Step 1. Necessary admissibility conditions for $b, c, \gamma, m$. In order to derive the particular form of $F$ and $R$ with the above parameter restrictions, we follow the 
approach of Keller-Ressel [35, Theorem 2.6]. Note that the $t$-derivative of $P_{t} e^{-\langle u, x\rangle}$ at $t=0$ exists for all $x, u \in S_{d}^{+}$, since

$$
\begin{aligned}
\lim _{t \rightarrow 0^{+}} \frac{P_{t} e^{-\langle u, x\rangle}-e^{-\langle u, x\rangle}}{t} & =\lim _{t \rightarrow 0^{+}} \frac{e^{-\phi(t, u)-\langle\psi(t, u), x\rangle}-e^{-\langle u, x\rangle}}{t} \\
& =(-F(u)-\langle R(u), x\rangle) e^{-\langle u, x\rangle}
\end{aligned}
$$

is well-defined by Proposition 3.4. Moreover, we can also write

$$
\begin{aligned}
-F(u)-\langle R(u), x\rangle & =\lim _{t \rightarrow 0^{+}} \frac{P_{t} e^{-\langle u, x\rangle}-e^{-\langle u, x\rangle}}{t e^{-\langle u, x\rangle}} \\
& =\lim _{t \rightarrow 0^{+}} \frac{1}{t}\left(\int_{S_{d}^{+} \backslash\{0\}} e^{-\langle u, \xi-x\rangle} p_{t}(x, d \xi)-1\right) \\
& =\lim _{t \rightarrow 0^{+}}\left(\frac{1}{t} \int_{S_{d}^{+}-x}\left(e^{-\langle u, \xi\rangle}-1\right) p_{t}(x, d \xi+x)+\frac{p_{t}\left(x, S_{d}^{+}\right)-1}{t}\right) .
\end{aligned}
$$

By the above equalities and the fact that $p_{t}\left(x, S_{d}^{+}\right) \leq 1$, we then obtain for $u=0$

$$
0 \geq \lim _{t \rightarrow 0^{+}} \frac{p_{t}\left(x, S_{d}^{+}\right)-1}{t}=-F(0)-\langle R(0), x\rangle .
$$

Setting $F(0)=c$ and $R(0)=\gamma$ yields $c \in \mathbb{R}^{+}$as in (2.5) and $\gamma \in S_{d}^{+}$as in (2.6). We thus obtain

$$
-(F(u)-c)-\langle R(u)-\gamma, x\rangle=\lim _{t \rightarrow 0^{+}} \frac{1}{t} \int_{S_{d}^{+}-x}\left(e^{-\langle u, \xi\rangle}-1\right) p_{t}(x, d \xi+x) .
$$

For every fixed $t>0$, the right hand side of (4.4) is the logarithm of the Laplace transform of a compound Poisson distribution supported on $S_{d}^{+}-\mathbb{R}_{+} x$ with intensity $p_{t}\left(x, S_{d}^{+}\right) / t$ and compounding distribution $p_{t}(x, d \xi+x) / p_{t}\left(x, S_{d}^{+}\right)$. Concerning the support, note that the compounding distribution is concentrated on $S_{d}^{+}-x$, which implies that the compound Poisson distribution has support on the convex cone $S_{d}^{+}-\mathbb{R}_{+} x$. By Lemma 4.5 the pointwise convergence of (4.4) for $t \rightarrow 0$ to some function being continuous at 0 , implies weak convergence of the compound Poisson distributions to some infinitely divisible probability distribution $K(x, d y)$ supported on $S_{d}^{+}-\mathbb{R}_{+} x$. Indeed, this follows from the fact that any compound Poisson distribution is infinitely divisible and the class of infinitely divisible distributions is closed under weak convergence ([47, Lemma 7.8]). Again, by Lemma 4.5 the Laplace transform of $K(x, d y)$ is then given as exponential of the left hand side of (4.4).

In particular, for $x=0, K(0, d y)$ is an infinitely divisible distribution with support on the cone $S_{d}^{+}$. By the Lévy-Khintchine formula on proper cones (see [49, Theorem 3.21]), its Laplace transform is therefore of the form

$$
\exp \left(-\langle b, u\rangle+\int_{S_{d}^{+} \backslash\{0\}}\left(e^{-\langle u, \xi\rangle}-1\right) m(d \xi)\right),
$$

where $b \in S_{d}^{+}$and $m$ is a Borel measure supported on $S_{d}^{+}$such that

$$
\int_{S_{d}^{+} \backslash\{0\}}(\|\xi\| \wedge 1) m(d \xi)<\infty
$$


yielding (2.7). Therefore,

$$
F(u)=\langle b, u\rangle+c-\int_{S_{d}^{+} \backslash\{0\}}\left(e^{-\langle u, \xi\rangle}-1\right) m(d \xi) .
$$

Step 2. Necessary admissibility conditions for $\beta^{i j}, \mu$. We next obtain the particular form of $R$. Observe that for each $x \in S_{d}^{+}$and $k \in \mathbb{N}$,

$$
\exp (-(F(u)-c) / k-\langle R(u)-\gamma, x\rangle)
$$

is the Laplace transform of the infinitely divisible distribution $K(k x, d y)^{* \frac{1}{k}}$, where $* \frac{1}{k}$ denotes the $\frac{1}{k}$ convolution power. For $k \rightarrow \infty$, these Laplace transforms obviously converge to $\exp (-\langle R(u)-\gamma, x\rangle)$ pointwise in $u$. Using again the same arguments as before (an application of Lemma 4.5 as below equation (4.4) $) K(k x, d y)^{* \frac{1}{k}}$ converges weakly to some infinitely divisible distribution $L(x, d y)$ on $S_{d}^{+}-\mathbb{R}_{+} x$ with Laplace transform $\exp (-\langle R(u)-\gamma, x\rangle)$ for $u \in S_{d}^{+}$.

By the Lévy-Khintchine formula on $S_{d}$ ([47, Theorem 8.1], indeed on $\mathbb{R}^{(d(d+1) / 2)}$ by modifying the scalar product appropriately), the characteristic function of $L(x, d y)$ has the form

$$
\widehat{L}(x, u)=e^{\frac{1}{2}\langle u, A(x) u\rangle+\langle B(x), u\rangle+\int_{S_{d} \backslash\{0\}}\left(e^{-\langle u, \xi\rangle}-1-\langle\chi(\xi), u\rangle\right) M(x, d \xi)}, \quad u \in \mathrm{i} S_{d},
$$

where $A(x)$ is a symmetric positive semidefinite linear operator on $S_{d}, B(x) \in S_{d}$, $M(x, \cdot)$ a measure on $S_{d} \backslash\{0\}$ satisfying

$$
\int_{S_{d} \backslash\{0\}}\left(\|\xi\|^{2} \wedge 1\right) M(x, d \xi)<\infty,
$$

and $\chi$ some appropriate truncation function. Furthermore, by [47, Theorem 8.7],

$$
\int_{S_{d} \backslash\{0\}} f(\xi) \frac{1}{t} p_{t}(x, d \xi+x) \stackrel{t \rightarrow 0}{\longrightarrow} \int_{S_{d} \backslash\{0\}} f(\xi) m(d \xi)+\int_{S_{d} \backslash\{0\}} f(\xi) M(x, d \xi)
$$

holds true for all $f: S_{d} \rightarrow \mathbb{R}$ which are bounded, continuous and vanishing on a neighborhood of 0 . We conclude that $M(x, d \xi)$ has support in $S_{d}^{+}-x$. Therefore, the characteristic function $\widehat{L}(x, u)$ admits an analytic extension to $S_{d}^{+} \times \mathrm{i} S_{d}$, which then has to coincide with the Laplace transform for $u \in S_{d}^{+}$. We conclude that, for all $x \in S_{d}^{+}$,

$$
\begin{aligned}
-\langle R(u)-\gamma, x\rangle & =\frac{1}{2}\langle u, A(x) u\rangle-\langle B(x), u\rangle \\
& +\int_{S_{d} \backslash\{0\}}\left(e^{-\langle u, \xi\rangle}-1+\langle\chi(\xi), u\rangle\right) M(x, d \xi), \quad u \in S_{d}^{+} .
\end{aligned}
$$

As the left side of (4.6) is linear in the components of $x$, it follows that $x \mapsto A(x)$, $x \mapsto B(x)$ as well as $x \mapsto \int_{E}\left(\|\xi\|^{2} \wedge 1\right) M(x, d \xi)$ for every $E \in \mathcal{B}\left(S_{d} \backslash\{0\}\right)$ are homogeneous additive maps on $S_{d}^{+}$in the sense of Lemma 4.4 This then implies that they are restrictions of linear maps on $S_{d}$, such that we can write

$$
\begin{gathered}
A(x)=\sum_{i, j} a_{i j} x_{i j}, \quad B(x)=\sum_{i, j} \beta^{i j} x_{i j}, \\
\int_{E}\left(\|\xi\|^{2} \wedge 1\right) M(x, d \xi)=\langle x, \mu(E)\rangle=\sum_{i, j} \mu_{i j}(E) x_{i j},
\end{gathered}
$$


where (recall that $c^{i j}$ denotes the standard basis of $S_{d}$ defined in Section 1.2):

$$
\begin{gathered}
a_{i j}=a_{j i}=\left(1+\delta_{i j}\right) \frac{A\left(c^{i j}\right)}{2}: S_{d} \rightarrow S_{d} \quad \text { linear, } \\
\beta^{i j}=\beta^{j i}=\left(1+\delta_{i j}\right) \frac{B\left(c^{i j}\right)}{2} \in S_{d}, \quad \text { and } \\
E \mapsto \mu_{i j}(E)=\mu_{j i}(E)=\left(1+\delta_{i j}\right) \frac{\int_{E}\left(\|\xi\|^{2} \wedge 1\right) M\left(c^{i j}, d \xi\right)}{2}
\end{gathered}
$$

are finite signed measures on $S_{d} \backslash\{0\}$. The fact that $M(x, \cdot)$ is a nonnegative measure for each $x \in S_{d}^{+}$implies immediately that $\mu(E)$ is a positive semidefinite matrix.

In (4.5), take now $x=\frac{1}{n} e^{i j}$ and $f=f_{n}=0$ on $S_{d}^{+}-\frac{1}{n} e^{i j}$ and strictly positive everywhere else. Then for each $n$ the left side of (4.5) is zero since the $p_{t}\left(\frac{1}{n} e^{i j}, d \xi+\right.$ $\left.\frac{1}{n} e^{i j}\right)$ is concentrated on $S_{d}^{+}-\frac{1}{n} e^{i j} . \operatorname{As} \operatorname{supp}(m) \subseteq S_{d}^{+}$, the first integral on the right vanishes as well. Hence,

$$
\begin{aligned}
0 & =\int_{S_{d} \backslash\{0\}} f_{n}(\xi) M\left(\frac{1}{n} e^{i j}, d \xi\right)=\int_{S_{d} \backslash\{0\}} \frac{f_{n}(\xi)}{\|\xi\|^{2} \wedge 1}\left\langle\frac{1}{n} e^{i j}, \mu(d \xi)\right\rangle \\
& =\frac{1}{n} \int_{S_{d} \backslash\{0\}} \frac{f_{n}(\xi)}{\|\xi\|^{2} \wedge 1}\left(\mu_{i i}(d \xi)+\left(1-\delta_{i j}\right)\left(\mu_{j j}(d \xi)+2 \mu_{i j}(d \xi)\right)\right.
\end{aligned}
$$

implies that $\operatorname{supp}\left(\mu_{i j}\right) \subseteq S_{d}^{+}-\frac{1}{n} e^{i j}$ for each $n$. Thus, we can conclude that $\operatorname{supp} \mu_{i j} \subseteq S_{d}^{+}$for all $1 \leq i, j \leq d$.

Now let $T: S_{d} \rightarrow S_{d}$ be any linear map with the property $T\left(S_{d}^{+}-\mathbb{R}_{+} x\right) \subseteq S_{d}^{+}$. Then $T(\operatorname{supp}(L(x, d y))) \subseteq S_{d}^{+}$. This implies that the pushforward $T_{*} L(x, \cdot)$ of $L(x, d y)$ under $T$ is an infinitely divisible distribution supported on $S_{d}^{+}$. By the Lévy-Khintchine formula on proper cones (see [49, Theorem 3.21]) and by [47, Proposition 11.10]) this implies that for all $x \in S_{d}^{+}$

$$
\begin{aligned}
& T A(x) T^{\top}=0, \\
& T B(x)+\int_{S_{d}^{+} \backslash\{0\}}(\widetilde{\chi}(T \xi)-T(\chi(\xi))) M(x, d \xi) \in S_{d}^{+}, \\
& \int_{S_{d}^{+} \backslash\{0\}}(\|\xi\| \wedge 1) T_{*} M(x, d \xi)<\infty,
\end{aligned}
$$

where $\tilde{\chi}$ denotes some truncation function associated with $T_{*} L(x, \cdot)$ and $T_{*} M$ the pushforward of $M$ under $T$. Due to (4.9) we can set $\tilde{\chi}=0$. Thus, (4.8) becomes

$$
T B(x)-\int T(\chi(\xi)) M(x, d \xi) \in S_{d}^{+}
$$

Moreover, equations (4.7), (4.10) and (4.9) are equivalent to

$$
\begin{aligned}
\left\langle T^{\top} v, A(x) T^{\top} v\right\rangle=0 & \text { for all } v \in S_{d}, \\
\left\langle B(x), T^{\top} v\right\rangle-\int_{S_{d}^{+} \backslash\{0\}}\left\langle(\chi(\xi)), T^{\top} v\right\rangle M(x, d \xi) \geq 0 & \text { for all } v \in S_{d}^{+}, \\
\int_{S_{d}^{+} \backslash\{0\}}(\|T \xi\| \wedge 1) M(x, d \xi)<\infty . &
\end{aligned}
$$


In particular, we claim that

$$
\begin{array}{rr}
\langle u, A(x) u\rangle=0, & \text { for all } u \in S_{d} \quad \text { s.t. } u x=x u=0, \\
\langle B(x), u\rangle-\int_{S_{d}^{+} \backslash\{0\}}\langle\chi(\xi), u\rangle M(x, d \xi) \geq 0, & \text { for all } u \in S_{d}^{+} \quad \text { s.t. } u x=x u=0, \\
\int_{S_{d}^{+} \backslash\{0\}}\langle\chi(\xi), u\rangle M(x, d \xi)<\infty, & \text { for all } u \in S_{d}^{+} \quad \text { s.t. } u x=x u=0 .
\end{array}
$$

Indeed, if $x$ is invertible then $u x=0$ is equivalent to $u=0$ and the assertions are obvious. Otherwise, if $x$ is in $\partial S_{d}^{+}$, the linear map $T_{u}$ defined in Lemma 4.3 is selfadjoint and satisfies $T_{u}\left(S_{d}^{+}-\mathbb{R}_{+} x\right) \subseteq S_{d}^{+}$. Furthermore, by Lemma 4.3](ii), there exists an element $v \in S_{d}$ such that $T_{u} v=u$. Hence, $\langle u, A(x) u\rangle=\left\langle T_{u}^{\top} v, A(x) T_{u}^{\top} v\right\rangle=$ 0. It follows from the proof of Lemma 4.3 that for $u \in S_{d}^{+}, v$ is an element of $S_{d}^{+}$ as well and we have $\langle B(x), u\rangle=\left\langle B(x), T_{u}^{\top} v\right\rangle$ and $\langle\chi(\xi), u\rangle=\left\langle(\chi(\xi)), T^{\top} v\right\rangle$. Equation (4.14) is obtained by choosing $T=T_{\sqrt{u}}$ in (4.11). This then leads

$$
\begin{aligned}
\int_{S_{d}^{+} \cap\{\|\xi\| \leq 1\}}\langle\xi, u\rangle M(x, d \xi) & =\int_{S_{d}^{+} \cap\{\|\xi\| \leq 1\}}\left\langle I_{d}, \xi u\right\rangle M(x, d \xi) \\
& \leq\left\|I_{d}\right\| \int_{S_{d}^{+} \cap\{\|\xi\| \leq 1\}}\|\xi u\| M(x, d \xi) \\
& =\left\|I_{d}\right\| \int_{S_{d}^{+} \cap\{\|\xi\| \leq 1\}}\left\|T_{\sqrt{u}} \xi\right\| M(x, d \xi)<\infty .
\end{aligned}
$$

From these arguments and Lemma 4.1, properties (2.11) and (2.9) can be derived so far. Thus, only (2.3) remains to be shown.

Step 3. Necessary admissibility condition for $\alpha$.

Due to the linearity of $A(x),\langle u, A(x) u\rangle$ can be written as $4\langle x, \vartheta(u)\rangle$, where the $(i j)^{t h}$ component of $\vartheta(u) \in S_{d}$ is defined by $\vartheta_{i j}(u)=1 / 4\left\langle u, a_{i j} u\right\rangle$. Note that $\vartheta$ is defined on all of $S_{d}$. Given that for all $x \in S_{d}^{+}, A(x)$ is a positive semidefinite operator on $S_{d},\langle u, A(x) u\rangle \geq 0$ for all $u \in S_{d}$ and therefore, by the self duality of $S_{d}^{+}, \vartheta(u) \in S_{d}^{+}$. By (4.12), we have for all $u$ such that $u x=x u=0$

$$
0=\langle u, A(x) u\rangle=4\langle x, \vartheta(u)\rangle .
$$

Next, we show that $\vartheta$ is quasi-constant, that is, $\langle x, \vartheta(u+w)-\vartheta(u)\rangle=0$ for all $x, u, w \in S_{d}^{+}$with $\langle x, w\rangle=0$ (see Definition 4.7). Indeed, pick $x, u, w \in S_{d}^{+}$ with $\langle x, w\rangle=0$. According to our assumptions, $A(x) w=0$, due to (4.15) and the positivity of $A$. Hence,

$$
\begin{aligned}
4\langle x, \vartheta(u+w)-\vartheta(u)\rangle & =\langle u+w, A(x)(u+w)\rangle-\langle u, A(x) u\rangle \\
& =\langle u, A(x) w\rangle+\langle A(x) w, u\rangle=0,
\end{aligned}
$$

where the second last equality holds in view of the symmetry of $A(x)$.

We now claim that there exists some $\alpha \in S_{d}^{+}$such that $\vartheta(u)=u \alpha u$, for each $u \in$ $S_{d}$. It is sufficient to show that this statements holds for all orthogonal projectors $p \in S_{d}^{+}$, i.e., $\alpha \in S_{d}^{+}$such that $\vartheta(p)=p \alpha p$ for all orthogonal projectors $p$. Indeed, if this is the case, we can derive the general statement in the following way: take $u \in$ 
$S_{d}^{+}$, then - by spectral decomposition - there are numbers $\lambda_{i} \geq 0$ and orthogonal projectors $p_{i}$, which are mutually orthogonal, such that $u=\sum_{i=1}^{d} \lambda_{i} p_{i}$ (see e.g. Kato [34, Section I.6.9]). Since the assertion holds for all orthogonal projectors, we have that

$$
2 \vartheta(u)=\sum_{i, j=1}^{d} \lambda_{i} \lambda_{j}\left(\vartheta\left(p_{i}+p_{j}\right)-\vartheta\left(p_{i}\right)-\vartheta\left(p_{j}\right)\right)
$$

by the property that $\vartheta$ is quadratic. Since $p_{i}+p_{j}$ is again an orthogonal projector we obtain the result.

We prove the assertion on orthogonal projectors by quasi-constancy. Take an arbitrary orthogonal projector $p$ and define $q=I_{d}-p$. Additionally we define $\alpha=\vartheta\left(I_{d}\right)$. By quasi-constancy we obtain

$$
\langle x, \vartheta(p+q)-\vartheta(q)\rangle=\langle y, \vartheta(p+q)-\vartheta(p)\rangle=0,
$$

and

$$
\langle x, \vartheta(p)\rangle=\langle y, \vartheta(q)\rangle=0
$$

for all $x, y \in S_{d}^{+}$with $\langle x, p\rangle=0$ and $\langle y, q\rangle=0$. Therefore $\alpha-\vartheta(q)$ and $\vartheta(p)$ are orthogonal to the orthogonal complement of $p$ in $S_{d}^{+}$(i.e., the positive symmetric matrices of the form $q u q$ by Lemma 4.2), and $\alpha-\vartheta(p)$ and $\vartheta(q)$ are orthogonal to the orthogonal complement of $q$ in $S_{d}^{+}$(the positive symmetric matrices of the form pup by Lemma 4.2). This means that we can write

$$
\alpha=\vartheta(p)+\vartheta(q)+\beta,
$$

where the symmetric matrix $\beta$ is orthogonal to all elements which are orthogonal to $p$ and $q$ (in $S_{d}^{+}$), i.e., $\beta$ is orthogonal to the linear span of matrices of the form pup and quq. However, such a decomposition is unique, since all vectors in the sum are mutually orthogonal, and the decomposition is given by

$$
\alpha=(p+q) \alpha(p+q)=p \alpha p+q \alpha q+(p \alpha q+q \alpha p) .
$$

Therefore we can conclude the assertion $\vartheta(p)=p \alpha p$. Since $p$ was arbitrary the assertion is proved.

Finally, all the derived restrictions on the parameters together with (4.6) then yield (2.17).

4.2. Infinitesimal Generator. The aim of this section is to prove the form of the infinitesimal generator as stated in (2.12).

Proposition 4.11. The infinitesimal generator $\mathcal{A}$ of an affine process on $S_{d}^{+}$satisfies $\mathcal{S}_{+} \subset \mathcal{D}(\mathcal{A})$ and is of the form (2.12) for all $f \in \mathcal{S}_{+}$and $x \in S_{d}^{+}$.

Proof. As already mentioned in the proof of Proposition 4.9, the $t$-derivative of $P_{t} e^{-\langle u, x\rangle}$ at $t=0$ exists pointwise for all $x, u \in S_{d}^{+}$and is given by (4.3). Furthermore, $x \mapsto(-F(u)-\langle R(u), x\rangle) e^{-\langle u, x\rangle} \in C_{0}\left(S_{d}^{+}\right)$, for $u \in S_{d}^{++}$. As $\left(P_{t}\right)$ is a Feller semigroup on $C_{0}\left(S_{d}^{+}\right)$, it follows from [47, Lemma 31.7] that $\left\{e^{-\langle u, x\rangle} \mid u \in S_{d}^{++}\right\} \in$ $D(\mathcal{A})$ and

$$
\mathcal{A} e^{-\langle u, x\rangle}=(-F(u)-\langle R(u), x\rangle) e^{-\langle u, x\rangle} .
$$


Combined with Proposition 4.9 we thus obtain

$$
\begin{aligned}
& \mathcal{A} e^{-\langle u, x\rangle}=\left(-\langle b, u\rangle-c+\int_{S_{d}^{+} \backslash\{0\}}\left(e^{-\langle u, \xi\rangle}-1\right) m(d \xi)\right. \\
& \left.+\left\langle 2 u \alpha u-B^{\top}(u)-\gamma+\int_{S_{d}^{+} \backslash\{0\}}\left(\frac{e^{-\langle u, \xi\rangle}-1+\langle\chi(\xi), u\rangle}{\|\xi\|^{2} \wedge 1}\right) \mu(d \xi), x\right\rangle\right) e^{-\langle u, x\rangle} \\
& =\frac{1}{2} \sum_{i, j, k, l} A_{i j k l}(x) u_{i j} u_{k l} e^{-\langle u, x\rangle}+\left\langle b+B(x), \nabla e^{-\langle u, x\rangle}\right\rangle-(c+\langle\gamma, x\rangle) e^{-\langle u, x\rangle} \\
& +\int_{S_{d}^{+} \backslash\{0\}}\left(e^{-\langle u, x+\xi\rangle}-e^{-\langle u, x\rangle}\right) m(d \xi) \\
& +\int_{S_{d}^{+} \backslash\{0\}}\left(e^{-\langle u, x+\xi\rangle}-e^{-\langle u, x\rangle}+\left\langle\chi(\xi), \nabla e^{-\langle u, x\rangle}\right\rangle\right) M(x, d \xi) .
\end{aligned}
$$

Indeed, in order to obtain the form of the diffusion part, observe that we have by symmetrization

$$
\begin{aligned}
2\langle u \alpha u, x\rangle & =2 \sum_{i, j, k, l} \alpha_{j k} x_{i l} u_{i j} u_{k l} \\
& =\frac{1}{2} \sum_{i, j, k, l}\left(x_{i k} \alpha_{j l}+x_{i l} \alpha_{j k}+x_{j k} \alpha_{i l}+x_{j l} \alpha_{i k}\right) u_{i j} u_{k l} \\
& =\frac{1}{2} \sum_{i, j, k, l} A_{i j k l}(x) u_{i j} u_{k l},
\end{aligned}
$$

see (2.13).

According to Theorem B.3 the linear hull $\mathcal{M}$ of $\left\{e^{-\langle u, \cdot\rangle} \mid u \in S_{d}^{++}\right\}$is dense in $\mathcal{S}_{+}$with respect to the family of seminorms $p_{k,+}$ defined in (B.2). Denoting the right hand side of (2.12) by $\mathcal{A}^{\sharp}$, we now claim that for every $f \in \mathcal{S}_{+}$

$$
\lim _{n \rightarrow \infty}\left\|\mathcal{A}^{\sharp} f_{n}-\mathcal{A}^{\sharp} f\right\|_{\infty}=0,
$$

where $f_{n} \in \mathcal{M}$ such that $\lim _{n \rightarrow \infty} p_{k,+}\left(f-f_{n}\right)=0$ for every $k$. Indeed, this is obvious for the differential operator part of $\mathcal{A}^{\sharp}$. By choosing $\chi(\xi)=1_{\{\|\xi\| \leq 1\}} \xi$ and by denoting $g(x):=f_{n}(x)-f(x)$, we obtain the following estimate for the integral 
part

$$
\begin{aligned}
& \left\|\int_{S_{d}^{+} \backslash\{0\}}\left(\frac{g(x+\xi)-g(x)-\left\langle 1_{\{\|\xi\| \leq 1\}} \xi, \nabla g(x)\right\rangle}{\|\xi\|^{2} \wedge 1}\right) x_{i j} \mu_{i j}(d \xi)\right\|_{\infty} \\
& \leq \int_{S_{d}^{+} \backslash\{0\} \cap\{\|\xi\| \leq 1\}}\left\|\left(\sum_{k, l, m, n}\left(\int_{0}^{1} \frac{\partial g(x+s \xi)}{\partial x_{k l} \partial x_{m n}}(1-s) d s\right) \frac{\xi_{k l} \xi m n}{\|\xi\|^{2}}\right) x_{i j}\right\|_{\infty} \\
& \times\left(\mu_{i j}^{+}(d \xi)+\mu_{i j}^{-}(d \xi)\right) \\
& +\int_{S_{d}^{+} \backslash\{0\} \cap\{\|\xi\|>1\}}\left\|(g(x+\xi)-g(x)) x_{i j}\right\|_{\infty}\left(\mu_{i j}^{+}(d \xi)+\mu_{i j}^{-}(d \xi)\right) \\
& \leq C_{1} p_{3,+}(g) \int_{S_{d}^{+} \backslash\{0\} \cap\{\|\xi\| \leq 1\}} \frac{\|\xi\|^{2}}{\|\xi\|^{2}}\left(\mu_{i j}^{+}(d \xi)+\mu_{i j}^{-}(d \xi)\right) \\
& +\int_{S_{d}^{+} \backslash\{0\} \cap\{\|\xi\|>1\}}\left(\|g(x+\xi)(\|x+\xi\|)\|_{\infty}+\left\|g(x) x_{i j}\right\|_{\infty}\right)\left(\mu_{i j}^{+}(d \xi)+\mu_{i j}^{-}(d \xi)\right) \\
& \leq C_{2} p_{3,+}(g)\left(\mu_{i j}^{+}\left(S_{d}^{+}\right)+\mu_{i j}^{-}\left(S_{d}^{+}\right)\right) \leq C_{3} p_{3,+}(g)
\end{aligned}
$$

where $C_{1}, C_{2}$ and $C_{3}$ denote some constants and $\mu_{i j}^{+}, \mu_{i j}^{-}$correspond to the Jordan decomposition $\mu_{i j}=\mu_{i j}^{+}-\mu_{i j}^{-}$. In the second last inequality we use the estimate $x_{i j} \leq\|x+\xi\|$. The same as above can be shown for the measure $m(d \xi)$, whence (4.17) holds true. As by the first part of the proof we have $\mathcal{A}^{\sharp}=\mathcal{A}$ for all elements of $\mathcal{M}$, 4.17) implies

$$
\lim _{n \rightarrow \infty}\left\|\mathcal{A} f_{n}-\mathcal{A}^{\sharp} f\right\|_{\infty}=0 .
$$

Since the infinitesimal generator of every Feller process is a closed operator, it follows that $\mathcal{S}_{+} \subset \mathcal{D}(\mathcal{A})$ and $\mathcal{A}=\mathcal{A}^{\sharp}$ on $\mathcal{S}_{+}$.

4.3. Linear Transformations and Canonical Representation. In this subsection we shall deal with linear transformations of affine processes. The proposition below states how the parameters of an affine process on $S_{d}^{+}$change under such linear maps, which allows us to establish a canonical representation of an affine process.

Proposition 4.12. Suppose $X$ is an affine process on $S_{d}^{+}$with parameters $\alpha, \beta^{i j}, c, \gamma$, $m, \mu$ as specified in Definition 2.3 and $b \in S_{d}^{+}$. Furthermore, let $G: S_{d}^{+} \rightarrow S_{\underline{d}}^{+}, x \mapsto$ $g x g^{\top}$ be an automorphism, where $g \in M_{d}$ is invertible. Then, $Y_{t}:=g X_{t} g^{\top}$ is an affine process on $S_{d}^{+}$, whose parameters, denoted by $\sim$, are given as follows with 
respect to the truncation function $\tilde{\chi}=g \chi\left(g^{-1} \xi\left(g^{\top}\right)^{-1}\right) g^{\top}$ :

$$
\begin{aligned}
\widetilde{b} & =g b g^{\top} \\
\widetilde{c} & =c, \\
\widetilde{m}(d \xi) & =G_{*} m(d \xi), \\
\widetilde{\alpha} & =g \alpha g^{\top}, \\
\widetilde{\gamma} & =\left(g^{\top}\right)^{-1} \gamma g^{-1}, \\
\widetilde{\mu}(d \xi) & =\left(\frac{\|\xi\|^{2} \wedge 1}{\left\|g^{-1} \xi\left(g^{\top}\right)^{-1}\right\|^{2} \wedge 1}\right)\left(g^{\top}\right)^{-1} G_{*} \mu(d \xi) g^{-1}, \\
\widetilde{B}^{\top}(u) & =\left(g^{\top}\right)^{-1} B^{\top}\left(g^{\top} u g\right) g^{-1},
\end{aligned}
$$

where $G_{*} m\left(G_{*} \mu\right)$ is the pushforward of the measure $m$ ( $\mu$ respectively).

Proof. Let us consider the process

$$
Y_{t}^{y}=g X_{t}^{g^{-1} y\left(g^{\top}\right)^{-1}} g^{\top},
$$

for which we have

$$
\begin{aligned}
& \mathbb{E}\left[\exp \left(-\left\langle u, Y_{t}^{y}\right\rangle\right)\right]=\mathbb{E}\left[\exp \left(-\left\langle u, g X_{t}^{g^{-1} y\left(g^{\top}\right)^{-1}} g^{\top}\right\rangle\right)\right] \\
& =\mathbb{E}\left[\exp \left(-\left\langle g^{\top} u g, X_{t}^{g^{-1} y\left(g^{\top}\right)^{-1}}\right\rangle\right)\right] \\
& =\exp \left(-\phi\left(t, g^{\top} u g\right)-\left\langle\psi\left(t, g^{\top} u g\right), g^{-1} y\left(g^{\top}\right)^{-1}\right\rangle\right) \\
& =\exp \left(-\phi\left(t, g^{\top} u g\right)-\left\langle\left(g^{\top}\right)^{-1} \psi\left(t, g^{\top} u g\right) g^{-1}, y\right\rangle\right) \text {. }
\end{aligned}
$$

Define now $\widetilde{\phi}$ and $\widetilde{\psi}$ by

$$
\widetilde{\phi}(t, u)=\phi\left(t, g^{\top} u g\right) \quad \text { and } \quad \widetilde{\psi}(t, u)=\left(g^{\top}\right)^{-1} \psi\left(t, g^{\top} u g\right) g^{-1},
$$

to see that $Y$ is an affine process on $S_{d}^{+}$. Using (2.14) and (2.16) we consequently obtain

$$
\begin{aligned}
\frac{\partial \widetilde{\phi}(t, u)}{\partial t} & =\frac{\partial \phi\left(t, g^{\top} u g\right)}{\partial t}=F\left(\psi\left(t, g^{\top} u g\right)\right) \\
& =\left\langle b, \psi\left(t, g^{\top} u g\right)\right\rangle+c-\int_{S_{d}^{+} \backslash\{0\}}\left(e^{-\left\langle\psi\left(t, g^{\top} u g\right), \xi\right\rangle}-1\right) m(d \xi) \\
& =\left\langle g b g^{\top}, \widetilde{\psi}(t, u)\right\rangle+c-\int_{S_{d}^{+} \backslash\{0\}}\left(e^{-\left\langle\widetilde{\psi}(t, u), g \xi g^{\top}\right\rangle}-1\right) m(d \xi) \\
& =\langle\widetilde{b}, \widetilde{\psi}(t, u)\rangle+c-\int_{S_{d}^{+} \backslash\{0\}}\left(e^{-\langle\widetilde{\psi}(t, u), \xi\rangle}-1\right) G_{*} m(d \xi) .
\end{aligned}
$$

Due to the uniqueness of the Lévy-Khintchine decomposition, this implies that $b$ transforms to $\widetilde{b}=g b g^{\top}, c$ remains constant and $m$ becomes $\widetilde{m}(d \xi)=G_{*} m(d \xi)$. For 
$\widetilde{\psi}$ we proceed similarly, that is we have

$$
\begin{aligned}
\frac{\partial \widetilde{\psi}(t, u)}{\partial t}= & \left(g^{\top}\right)^{-1} \frac{\partial \psi\left(t, g^{\top} u g\right)}{\partial t} g^{-1}=\left(g^{\top}\right)^{-1} R\left(\psi\left(t, g^{\top} u g\right)\right) g^{-1} \\
= & \left(g^{\top}\right)^{-1}\left(-\psi\left(t, g^{\top} u g\right) \alpha \psi\left(t, g^{\top} u g\right)+B^{\top}\left(\psi\left(t, g^{\top} u g\right)\right)\right. \\
& \left.+\gamma-\int_{S_{d}^{+} \backslash\{0\}}\left(\frac{e^{-\left\langle\psi\left(t, g^{\top} u g\right), \xi\right\rangle}-1+\left\langle\chi(\xi), \psi\left(t, g^{\top} u g\right)\right\rangle}{\|\xi\|^{2} \wedge 1}\right) \mu(d \xi)\right) g^{-1}
\end{aligned}
$$

from which it can be seen that $\alpha$ transforms to $\widetilde{\alpha}=g \alpha g^{\top}, \gamma$ becomes $\widetilde{\gamma}=$ $\left(g^{\top}\right)^{-1} \gamma g^{-1}$, and $\mu$ changes to

$$
\widetilde{\mu}(E)=\left(g^{\top}\right)^{-1}\left(\int_{E}\left(\frac{\|\xi\|^{2} \wedge 1}{\left\|g^{-1} \xi\left(g^{\top}\right)^{-1}\right\|^{2} \wedge 1}\right) G_{*} \mu(d \xi)\right) g^{-1}
$$

for every $E \in \mathcal{B}\left(S_{d}^{+} \backslash\{0\}\right)$. Moreover, since $\tilde{\chi}=g \chi\left(g^{-1} \xi\left(g^{\top}\right)^{-1}\right) g^{\top}$

$$
\widetilde{B}^{\top}(u)=\left(g^{\top}\right)^{-1} B^{\top}\left(g^{\top} u g\right) g^{-1} .
$$

By means of Proposition 4.12 we can derive a canonical representation for affine processes:

Proposition 4.13. Let $X$ be an affine process on $S_{d}^{+}$with parameters $\alpha, \beta^{i j}, c, \gamma$, $m, \mu$ as specified in Definition 2.3 and $b \in S_{d}^{+}$. Then there exists an automorphism $G: S_{d}^{+} \rightarrow S_{d}^{+}, x \mapsto g x g^{\top}$ such that the parameters of the affine process $Y=g X g^{\top}$, denoted by $\stackrel{\sim}{ }$, are as in Proposition 4.12 with

$$
\widetilde{b}=\theta=\operatorname{diag}\left(\theta_{11}, \ldots, \theta_{d d}\right), \quad \widetilde{\alpha}=I_{r}^{d},
$$

where we define

$$
I_{r}^{d}=\left(\begin{array}{cc}
I_{r} & 0 \\
0 & 0
\end{array}\right)
$$

Proof. By Proposition 4.12 the parameters of $Y=g X g^{\top}$ transform as

$$
\widetilde{\alpha}=g \alpha g^{\top}, \quad \widetilde{b}=g b g^{\top} .
$$

Since $\alpha$ and $b \in S_{d}^{+}$, they are jointly diagonalizable through an automorphism on $S_{d}^{+}$. More precisely, there exists an invertible matrix $g \in M_{d}$ such that

$$
g \alpha g^{\top}=I_{r}^{d} \quad \text { with } r=\operatorname{rk}(\alpha)
$$

and

$$
g b g^{\top}=\operatorname{diag}\left(\theta_{1}, \ldots, \theta_{d}\right)=: \theta,
$$

where rk denotes the rank of a matrix. For the proof of this fact, we refer to ([23, Theorem 8.7.1]). 
4.4. Condition on the Constant Drift. This subsection is devoted to show that condition (2.4) holds true for any affine process $X$ on $S_{d}^{+}$. Since the automorphism $G: S_{d}^{+} \rightarrow S_{d}^{+}$in Proposition 4.13 is order preserving, it suffices to consider affine processes of the canonical form as specified in Proposition 4.13. The following result is a consequence of the Lévy-Khintchine formula on $\mathbb{R}_{+}$.

Lemma 4.14. Let $Y$ be an affine process of canonical form as specified in Propo-

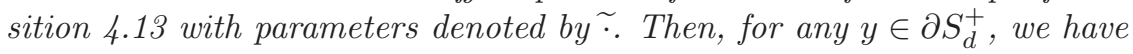

$$
\nabla \operatorname{det}(y) \in N_{S_{d}^{+}}(y), \quad \int_{S_{d}^{+} \backslash\{0\}}\langle\widetilde{\chi}(\xi), \nabla \operatorname{det}(y)\rangle \widetilde{M}(y, d \xi)<\infty
$$

and

$$
\begin{aligned}
\langle\theta, \nabla \operatorname{det}(y)\rangle+\langle\widetilde{B}(y), \nabla \operatorname{det}(y)\rangle-\int_{S_{d}^{+} \backslash\{0\}}\langle\widetilde{\chi}(\xi), \nabla \operatorname{det}(y)\rangle \widetilde{M}(y, d \xi) & \\
& +\frac{1}{2} \sum_{i, j, k, l} \widetilde{A}_{i j k l}(y) \partial_{i j} \partial_{k l} \operatorname{det}(y) \geq 0 .
\end{aligned}
$$

Proof. Let $y \in \partial S_{d}^{+}$and let $f \in C_{c}^{\infty}\left(S_{d}^{+}\right)$be a function with $f \geq 0$ and $f(x)=$ $\operatorname{det}(x)$ for all $x$ in a neighborhood of $y$. Then, for any $v \in \mathbb{R}_{+}$, the function $x \mapsto e^{-v f(x)}-1$ lies in $C_{c}^{\infty}\left(S_{d}^{+}\right)$and thus in $\mathcal{D}(\widetilde{\mathcal{A}})$, where $\widetilde{\mathcal{A}}$ denotes the infinitesimal generator of $Y$. Note that $f(y)=0$. Hence the limit

$\widetilde{\mathcal{A}}\left(e^{-v f(y)}-1\right)=\lim _{t \rightarrow 0^{+}} \frac{1}{t} \int_{S_{d}^{+}}\left(e^{-v f(\xi)}-1\right) \widetilde{p}_{t}(y, d \xi)=\lim _{t \rightarrow 0^{+}} \frac{1}{t} \int_{\mathbb{R}_{+}}\left(e^{-v z}-1\right) p_{t}^{f}(y, d z)$,

exists for any $v \in \mathbb{R}_{+}$, where $\widetilde{p}_{t}(y, d \xi)$ denotes the transition function of $Y$, and $p_{t}^{f}(y, d z)=f_{*} \widetilde{p}_{t}(y, d z)$ is the pushforward of $\widetilde{p}_{t}(y, \cdot)$ under $f$, which is a probability measure supported on $\mathbb{R}_{+}$.

Using the same arguments as in Proposition 4.9 (that is, applying Lemma 4.5 as done below equation (4.4) ), and noting that $f(y)=0$, we conclude that

$$
\begin{aligned}
v \mapsto & \widetilde{\mathcal{A}}\left(e^{-v f(y)}-1\right) \\
= & \frac{1}{2} \sum_{i, j, k, l} \widetilde{A}_{i j k l}(y)\left(v^{2} \partial_{i j} f(y) \partial_{k l} f(y)-v \partial_{i j} \partial_{k l} f(y)\right)-v\langle\theta+\widetilde{B}(y), \nabla f(y)\rangle \\
& +\int_{S_{d}^{+} \backslash\{0\}}\left(e^{-v f(y+\xi)}-1\right) \widetilde{m}(y, d \xi) \\
& +\int_{S_{d}^{+} \backslash\{0\}}\left(e^{-v f(y+\xi)}-1+v\langle\widetilde{\chi}(\xi), \nabla f(y)\rangle\right) \widetilde{M}(y, d \xi)
\end{aligned}
$$

is the logarithm of the Laplace transform of an infinitely divisible distribution on $\mathbb{R}_{+}$. Note that

$$
\langle\nabla \operatorname{det}(y), x\rangle=\left.\frac{d}{d t} \operatorname{det}(y+t x)\right|_{t=0} \begin{cases}\geq 0, & x \in S_{d}^{+} \\ =0, & x=y .\end{cases}
$$

Hence $\nabla \operatorname{det}(y) \in N_{S_{d}^{+}}(y)$ and the admissibility condition (2.9) implies (4.19). By the Lévy-Khintchine formula on $\mathbb{R}_{+}$(see [49, Theorem 3.21]), the linear coefficient in $v$ in (4.21) has to be nonpositive. But this is now just (4.20), whence the lemma is proved. 
It now remains to show that (2.4) follows from (4.20). For this purpose it suffices to evaluate (4.20) at diagonal elements $y \in \partial S_{d}^{+}$. Thus, we state the following lemma.

Lemma 4.15. Let $y \in S_{d}^{+}$be diagonal, and let $f \in C_{c}^{2}\left(S_{d}^{+}\right)$. Then we have

$$
\begin{aligned}
& \left.\frac{1}{2} \sum_{i, j, k, l=1}^{d}\left(y_{i k}\left(I_{r}^{d}\right)_{j l}+y_{i l}\left(I_{r}^{d}\right)_{j k}+y_{j k}\left(I_{r}^{d}\right)_{i l}+y_{j l}\left(I_{r}^{d}\right)_{i k}\right) \frac{\partial^{2} f(x)}{\partial x_{i j} \partial x_{k l}}\right|_{x=y} \\
& =\frac{1}{2} \sum_{i, j=1}^{d}\left(y_{i i} 1_{\{j \leq r\}}+y_{j j} 1_{\{i \leq r\}}\right)\left(\left.\frac{\partial^{2} f(x)}{\partial x_{i j}^{2}}\right|_{x=y}+\left.\frac{\partial^{2} f(x)}{\partial x_{i j} x_{j i}}\right|_{x=y}\right) .
\end{aligned}
$$

Proof. Obvious.

Next, we calculate the partial derivatives of the determinant.

Lemma 4.16. Let $y \in S_{d}^{+}$be diagonal, $y=\operatorname{diag}\left(y_{11}, y_{22}, \ldots, y_{d d}\right)$. Then,

$$
\left.\frac{\partial \operatorname{det}(x)}{\partial x_{i j}}\right|_{x=y}= \begin{cases}\prod_{k \neq i} y_{k k} & \text { if } i=j \\ 0 & \text { else }\end{cases}
$$

and

$$
\begin{array}{ll}
\left.\frac{\partial^{2} \operatorname{det}(x)}{\partial x_{i j} x_{j i}}\right|_{x=y}=-\prod_{k=1, k \neq i, k \neq j}^{d} y_{k k} & \text { for } 1 \leq i<j \leq d \\
\left.\frac{\partial^{2} \operatorname{det}(x)}{\partial x_{i j}^{2}}\right|_{x=y}=0 & \text { for } 1 \leq i \leq j \leq d
\end{array}
$$

hold true, where $\prod$ over 0 is defined to be 1.

Proof. In dimension $d=2$, the assertion is easily checked, as $\operatorname{det}(y)=y_{11} y_{22}-$ $y_{12} y_{21}$. Therefore, we have

$$
\partial_{11} \operatorname{det}(x)=y_{22}, \quad \partial_{22} \operatorname{det}(x)=y_{11}, \quad \partial_{12} \operatorname{det}(x)=\partial_{21} \operatorname{det}(x)=0
$$

as well as

$$
\begin{aligned}
& \partial_{11}^{2} \operatorname{det}(x)=\partial_{22}^{2} \operatorname{det}(x)=\partial_{12}^{2} \operatorname{det}(x)=\partial_{21}^{2} \operatorname{det}(x)=0 \\
& \partial_{12} \partial_{21} \operatorname{det}(x)=\partial_{21} \partial_{12} \operatorname{det}(x)=-1
\end{aligned}
$$

For dimension strictly larger than 2, we employ a combinatorial argument. Recall Leibniz's definition of the determinant,

$$
\operatorname{det}(x)=\sum_{\sigma \in \Sigma} \operatorname{sgn}(\sigma) \prod_{k=1}^{d} x_{k \sigma(k)}
$$

where $\sigma$ is an element of the permutation group $\Sigma$ on the set $\{1,2, \ldots, d\}$ and $\operatorname{sgn}$ denotes the signum function on $\Sigma$, that is $\operatorname{sgn}=1$ if $\sigma$ is an even permutation and $s g n=-1$ if it is odd. Differentiation of (4.22) with respect to $x_{i j}$ yields

$$
\left.\frac{\partial \operatorname{det}(x)}{\partial x_{i j}}\right|_{x=y}=\left.\left(\sum_{\sigma \in \Sigma} \operatorname{sgn}(\sigma) 1_{\{\sigma(i)=j\}} \prod_{k \neq i} x_{k \sigma(k)}\right)\right|_{x=y}= \begin{cases}\prod_{k \neq i} y_{k k} & \text { if } i=j \\ 0 & \text { else. }\end{cases}
$$


Thus, for the second derivative we have

$$
\left.\frac{\partial^{2} \operatorname{det}(x)}{\partial x_{i j} \partial x_{j i}}\right|_{x=y}=\left.\left(\sum_{\sigma \in \Sigma} \operatorname{sgn}(\sigma) 1_{\{\sigma(i)=j\}} 1_{\{\sigma(j)=i\}} \prod_{k \neq i \neq j} x_{k \sigma(k)}\right)\right|_{x=y}=-\prod_{k \neq i \neq j} y_{k k},
$$

where the last equality holds since $y$ is diagonal. For $\partial_{i j}^{2} \operatorname{det}(x)$, the statement is obvious.

We are prepared to prove the admissibility condition on the constant drift,

Proposition 4.17. Let $X$ be an affine process on $S_{d}^{+}$, then (2.4) holds, that is

$$
b \succeq(d-1) \alpha .
$$

Proof. Since the automorphism $G: S_{d}^{+} \rightarrow S_{d}^{+}$in Proposition 4.13 is order preserving, it suffices to show that (4.20) in Lemma 4.14 implies

$$
\theta \succeq(d-1) I_{r}^{d} .
$$

We show that $\theta_{m m} \geq d-1$, if $r \geq m$. To this end, take again some diagonal $y \in \partial S_{d}^{+}$of form $y=\operatorname{diag}\left(y_{11}>0, \ldots, y_{m m}=0, \ldots, y_{d d}>0\right)$. By Lemma 4.15 and (4.16) we obtain

$$
\begin{aligned}
& \sum_{i=1}^{d} \theta_{i i} \partial_{i i} \operatorname{det}(y)+\sum_{i, j}(B(y))_{i j} \partial_{i j} \operatorname{det}(y) \\
& \quad-\int_{S_{d}^{+} \backslash\{0\}}\left(\sum_{i, j}(\widetilde{\chi}(\xi))_{i j} \partial_{i j} \operatorname{det}(x)\right) \widetilde{M}(y, d \xi) \\
& \quad+\frac{1}{2} \sum_{i, j=1}^{d}\left(\left(y_{i i} 1_{\{j \leq r\}}+y_{j j} 1_{\{i \leq r\}}\right)\left(\partial_{i j}^{2} \operatorname{det}(y)+\partial_{i j} \partial_{j i} \operatorname{det}(y)\right)\right) \\
& =\sum_{i=1}^{d}\left(\theta_{i i} \prod_{k \neq i} y_{k k}\right)+\sum_{l \neq m}\left(\beta_{m m}^{l l} y_{l l} \prod_{k \neq m} y_{k k}\right) \\
& \quad-\sum_{l \neq m} \int_{S_{d}^{+} \backslash\{0\}} \frac{(\widetilde{\chi}(\xi))_{m m} y_{l l} \prod_{k \neq m} y_{k k} \widetilde{\mu}_{l l}(d \xi)}{\|\xi\|^{2} \wedge 1} \\
& \quad-\frac{1}{2} \sum_{i \neq j}\left(\prod_{k \neq j} y_{k k} 1_{\{j \leq r\}}+\prod_{k \neq i} y_{k k} 1_{\{i \leq r\}}\right) \\
& =\theta_{m m} \prod_{k \neq m} y_{k k}+\prod_{k \neq m} y_{k k}\left(\sum_{l \neq m}\left(\beta_{m m}^{l l} y_{l l}-y_{l l} \int_{S_{d}^{+} \backslash\{0\}} \frac{(\widetilde{\chi}(\xi))_{m m}}{\|\xi\|^{2} \wedge 1} \widetilde{\mu}_{l l}(d \xi)\right)\right) \\
& \quad-(d-1) \prod_{k \neq m} y_{k k} 1_{\{m \leq r\}} \geq 0 .
\end{aligned}
$$

As $\prod_{k \neq m} y_{k k}>0$ and by (2.11) also

$$
\left(\beta_{m m}^{l l} y_{l l}-y_{l l} \int_{S_{d}^{+} \backslash\{0\}} \frac{(\widetilde{\chi}(\xi))_{m m}}{\|\xi\|^{2} \wedge 1} \widetilde{\mu}_{l l}(d \xi)\right) \geq 0
$$


for $l \neq m$, letting $y_{l l} \rightarrow 0, l \neq m$ yields $\theta_{m m} \geq d-1$ for $r \geq m$. Relabeling of indices then proves (4.23).

\section{Sufficient Conditions for the Existence and Uniqueness of Affine Processes}

In this section we prove that for a given admissible parameter set $\left(\alpha, b, \beta^{i j}, c, \gamma, m, \mu\right)$ satisfying the conditions of Definition [2.3. there exists a unique affine process on $S_{d}^{+}$whose infinitesimal generator $\mathcal{A}$ is of form (2.12). Our approach to derive this result is to consider the martingale problem for the operator $\mathcal{A}$. In order to prove uniqueness for this martingale problem, we shall need the following existence and uniqueness result for the generalized Riccati differential equations (2.14)-(2.15).

5.1. Generalized Riccati Differential Equations. We first derive some properties of the function $R$ given in (2.17).

Lemma 5.1. $R$ is analytic on $S_{d}^{++}$and quasi-monotone increasing on $S_{d}^{+}$.

Proof. That $R$ is analytic on $S_{d}^{++}$follows by dominated convergence (see e.g. [17, Lemma A.2]).

Now let $\delta>0$, and define

$$
\begin{aligned}
R^{\delta}(u)= & -2 u \alpha u+B^{\top}(u)+\gamma-\int_{\{\|\xi\| \geq \delta\}}\left(\frac{e^{-\langle u, \xi\rangle}-1+\langle\chi(\xi), u\rangle}{\|\xi\|^{2} \wedge 1}\right) \mu(d \xi) \\
= & -2 u \alpha u+\gamma+\left(B^{\top}(u)-\int_{\{\|\xi\| \geq \delta\}} \frac{\langle\chi(\xi), u\rangle}{\|\xi\|^{2} \wedge 1} \mu(d \xi)\right) \\
& +\int_{\{\|\xi\| \geq \delta\}}\left(\frac{1-e^{-\langle u, \xi\rangle}}{\|\xi\|^{2} \wedge 1}\right) \mu(d \xi) .
\end{aligned}
$$

Now, the map $u \mapsto-2 u \alpha u+\gamma$ is quasi-monotone increasing, as it is shown in Step 3 of the proof of Proposition 4.9. Furthermore, it follows from the admissibility condition (2.11) that

$$
u \mapsto B^{\top}(u)-\int_{\{\|\xi\| \geq \delta\}} \frac{\langle\chi(\xi), u\rangle}{\|\xi\|^{2} \wedge 1} \mu(d \xi)
$$

is a quasi-monotone increasing linear map on $S_{d}^{+}$. Finally, the quasi-monotonicity of

$$
u \mapsto \int_{\{\|\xi\| \geq \delta\}}\left(\frac{1-e^{-\langle u, \xi\rangle}}{\|\xi\|^{2} \wedge 1}\right) \mu(d \xi)
$$

is a consequence of the monotonicity of the exponential and that $\operatorname{supp}(\mu) \subseteq S_{d}^{+}$.

By dominated convergence, we have $\lim _{\delta \rightarrow 0} R^{\delta}(u)=R(u)$ pointwise for each $u \in$ $S_{d}^{+}$. Hence the quasi-monotonicity carries over to $R$. Indeed, choose $x, u, v \in S_{d}^{+}$ such that $u \preceq v$ and $\langle v-u, x\rangle=0$. Then we have for all $\delta,\left\langle R^{\delta}(v)-R^{\delta}(u), x\right\rangle \geq 0$. Thus

$$
\left\langle R^{\delta}(v)-R^{\delta}(u), x\right\rangle \rightarrow\langle R(v)-R(u), x\rangle \geq 0,
$$

as $\delta \rightarrow 0$, which proves that $R$ is quasi-monotone increasing.

Lemma 5.2. There exists a constant $K$ such that

$$
\langle u, R(u)\rangle \leq \frac{K}{2}\left(\|u\|^{2}+1\right), \quad u \in S_{d}^{+} .
$$


Proof. We may assume, without loss of generality, that the truncation function in Definition 2.3 takes the form $\chi(\xi)=1_{\{\|\xi\| \leq 1\}} \xi$ (otherwise adjust $B(u)$ accordingly). Then, for all $u \in S_{d}^{+}$we have,

$$
\begin{aligned}
R(u)= & -2 u \alpha u+B^{\top}(u)+\gamma-\int_{S_{d}^{+} \backslash\{0\} \cap\{\|\xi\| \leq 1\}} \underbrace{\left(\frac{e^{-\langle u, \xi\rangle}-1+\langle\xi, u\rangle}{\|\xi\|^{2}}\right)}_{\geq 0} \mu(d \xi) \\
& -\int_{S_{d}^{+} \backslash\{0\} \cap\{\|\xi\|>1\}}\left(e^{-\langle u, \xi\rangle}-1\right) \mu(d \xi) \\
\preceq & -2 u \alpha u+B^{\top}(u)+\gamma+\mu\left(S_{d}^{+} \cap\{\|\xi\|>1\}\right) \\
\preceq & B^{\top}(u)+\gamma+\mu\left(S_{d}^{+} \cap\{\|\xi\|>1\}\right),
\end{aligned}
$$

where we use that

$$
-\int_{S_{d}^{+} \backslash\{0\} \cap\{\|\xi\|>1\}}\left(e^{-\langle u, \xi\rangle}-1\right) \mu(d \xi) \preceq \int_{S_{d}^{+} \backslash\{0\} \cap\{\|\xi\|>1\}} \mu(d \xi) .
$$

Set now

$$
\bar{\gamma}:=\gamma+\mu\left(S_{d}^{+} \cap\{\|\xi\|>1\}\right) \in S_{d}^{+} .
$$

By (5.2), we obtain, for $u \in S_{d}^{+}$, that

$$
\langle u, R(u)\rangle \leq\left\langle u, B^{\top}(u)\right\rangle+\langle u, \bar{\gamma}\rangle,
$$

from which we derive the existence of a positive constant $K$ such equation (5.1) holds.

Here is our main existence and uniqueness result for the generalized Riccati differential equations (2.14)-2.15).

Proposition 5.3. For every $u \in S_{d}^{++}$there exists a unique global $\mathbb{R}_{+} \times S_{d}^{++}$valued solution $(\phi, \psi)$ of (2.14)-(2.15). Moreover, $\phi(t, u)$ and $\psi(t, u)$ are analytic in $(t, u) \in \mathbb{R}_{+} \times S_{d}^{++}$.

Proof. We only have to show that, for every $u \in S_{d}^{++}$, there exists a unique global $S_{d}^{++}$-valued solution $\psi$ of (2.15), as then $\phi$ is uniquely determined by integrating (2.14) and has the desired properties by admissibility of the parameter set.

Let $u \in S_{d}^{++}$. Since $R$ is analytic on $S_{d}^{++}$, standard ODE results (e.g. 15, Theorem 10.4.5]) yield there exists a unique local $S_{d}^{++}$-valued solution $\psi(t, u)$ of (2.15) for $t \in\left[0, t_{+}(u)\right)$, where

$$
t_{+}(u)=\lim \inf _{n \rightarrow \infty}\left\{t \geq 0 \mid\|\psi(t, u)\| \geq n \text { or } \psi(t, u) \in \partial S_{d}^{+}\right\} \leq \infty .
$$

It thus remains to show that $t_{+}(u)=\infty$. That $\psi(t, u)$, and hence $\phi(t, u)$, is analytic in $(t, u) \in \mathbb{R}_{+} \times S_{d}^{++}$then follows from [15, Theorem 10.8.2].

Since $R$ may not be Lipschitz continuous at $\partial S_{d}^{+}$(see Remark 5.4 below), we first have to regularize it. We thus define

$$
\widetilde{R}(u)=-2 u \alpha u+B^{\top}(u)+\gamma-\int_{S_{d}^{+} \backslash\{0\} \cap\{\|\xi\| \leq 1\}}\left(\frac{e^{-\langle u, \xi\rangle}-1+\langle\xi, u\rangle}{\|\xi\|^{2}}\right) \mu(d \xi)
$$


It then follows as in Lemmas 5.1 and 5.2 that $\widetilde{R}$ is quasi-monotone increasing on $S_{d}^{+}$ and that (5.1) holds for some constant $\widetilde{K}$. Moreover, $\widetilde{R}$ is analytic on $S_{d}$. Hence, for all $u \in S_{d}$, there exists a unique local $S_{d}$-valued solution $\widetilde{\psi}$ of

$$
\frac{\partial \widetilde{\psi}(t, u)}{\partial t}=\widetilde{R}(\widetilde{\psi}(t, u)), \quad \widetilde{\psi}(0, u)=u
$$

for all $t \in\left[0, \widetilde{t}_{+}(u)\right)$ with maximal lifetime

$$
\widetilde{t}_{+}(u)=\lim \inf _{n \rightarrow \infty}\{t \geq 0 \mid\|\widetilde{\psi}(t, u)\| \geq n\} \leq \infty .
$$

Volkmann's comparison theorem 4.8 now implies

$$
0 \preceq \widetilde{\psi}(t, u) \preceq \widetilde{\psi}(t, v) \quad t \leq \widetilde{t}_{+}(u) \wedge \widetilde{t}_{+}(v) \quad \text { for all } 0 \preceq u \preceq v .
$$

Hence from (5.1), we infer that for all $u \in S_{d}^{+}$and $t<\widetilde{t}_{+}(u)$,

$$
\partial_{t}\|\widetilde{\psi}(t, u)\|^{2}=2\left\langle\widetilde{\psi}(t, u), \partial_{t} \widetilde{\psi}(t, u)\right\rangle \leq \widetilde{K}\left(\|\widetilde{\psi}(t, u)\|^{2}+1\right) .
$$

Gronwall's inequality (e.g. [15, (10.5.1.3)]) implies

$$
\|\widetilde{\psi}(t, u)\|^{2} \leq e^{\widetilde{K} t}\left(\|u\|^{2}+1\right), \quad t<\widetilde{t}_{+}(u) .
$$

Hence $\widetilde{t}_{+}(u)=\infty$ for $u \in S_{d}^{+}$. Since $\widetilde{\psi}(t, u)$ is also analytic in $u$, Lemma 3.3 implies that $\widetilde{\psi}(t, u) \in S_{d}^{++}$for all $(t, u) \in \mathbb{R}_{+} \times S_{d}^{++}$.

We now carry this over to $\psi(t, u)$ and assume without loss of generality, as in the proof of Lemma 5.2, that the truncation function in Definition 2.3 takes the form $\chi(\xi)=1_{\{\|\xi\| \leq 1\}} \xi$. Then

$$
R(u)-\widetilde{R}(u)=-\int_{S_{d}^{+} \backslash\{0\} \cap\{\|\xi\|>1\}}\left(e^{-\langle u, \xi\rangle}-1\right) \mu(d \xi) \succeq 0, \quad u \in S_{d}^{+} .
$$

Hence, for $u \in S_{d}^{++}$and $t<t_{+}(u)$, we have

$$
\frac{\partial \widetilde{\psi}(t, u)}{\partial t}-\widetilde{R}(\widetilde{\psi}(t, u))=\frac{\partial \psi(t, u)}{\partial t}-R(\psi(t, u)) \preceq \frac{\partial \psi(t, u)}{\partial t}-\widetilde{R}(\psi(t, u)) .
$$

Theorem 4.8 thus implies

$$
\psi(t, u) \succeq \widetilde{\psi}(t, u) \in S_{d}^{++}, \quad t \in\left[0, t_{+}(u)\right) .
$$

Hence $t_{+}(u)=\liminf _{n \rightarrow \infty}\{t \geq 0 \mid\|\psi(t, u)\| \geq n\}$. Using (5.1) again, we now can show as for $\widetilde{\psi}$ that

$$
\|\psi(t, u)\|^{2} \leq e^{K t}\left(\|u\|^{2}+1\right), \quad t<t_{+}(u) .
$$

Hence $t^{+}(u)=\infty$, as desired.

Remark 5.4. Lemma 5.1 states that the admissibility of the parameters $\alpha, \beta^{i j}, \gamma, \mu$ implies quasi-monotonicity of $R$ on $S_{d}^{+} 9$ Moreover, quasi-monotonicity just means that $R$ is "inward pointing" close to the boundary $S_{d}^{+}$. Indeed, let $u, x \in S_{d}^{+}$with $\langle u, x\rangle=0$. Then $\langle R(u), x\rangle \geq\langle\gamma, x\rangle \geq 0$. Hence, if $R$ were Lipschitz continuous on $S_{d}^{+}$, a deterministic variant of Theorem A.5 would imply the invariance of $S_{d}^{+}$ with respect to 2.15) right away. However, the map $R$ might fail to be Lipschitz at $\partial S_{d}^{+}$(see the 1-dimensional counterexample [17, Example 9.3]), even though it is analytic on the interior $S_{d}^{++}$. Here quasi-monotonicty plays again a decisive

\footnotetext{
${ }^{9}$ We conjecture that the converse also holds: $R$ is quasi-monotone on $S_{d}^{+}$and $\operatorname{supp}(\mu) \subseteq S_{d}^{+}$if and only if the parameters $\alpha, \beta^{i j}, \gamma, \mu$ are admissible.
} 
role, since it means in fact that close to the boundary the vector fields have inwardpointing increments. This leads to the phenomenon that $\psi(t, u)$ stays away from the boundary $\partial S_{d}^{+}$for $u \in S_{d}^{++}$, which is of crucial importance in our analysis.

5.2. The Martingale Problem for $\mathcal{A}$. We are now prepared to study the martingale problem for the operator $\mathcal{A}$ given by (2.12). For the notion of martingale problems we refer to [18, Chapter 4]. We shall proceed in four steps. First, we approximate $\mathcal{A}$ by regular operators $\mathcal{A}^{\epsilon, \delta, n}$ on the function space $C_{c}^{\infty}\left(S_{d}^{+}\right)$. Second, using Theorem $\mathrm{A} .5$ below, we show that there exists a $S_{d}^{+}$-valued càdlàg solution of the martingale problem for $\mathcal{A}^{\epsilon, \delta, n}$. Third, a subsequence of these solutions is shown to converge to an $S_{d}^{+} \cup\{\Delta\}$-valued càdlàg solution of the martingale problem for $\mathcal{A}$. Finally, we show that this solution is unique, Markov and affine, as desired.

Note that we cannot employ Stroock's [50 seminal existence and uniqueness results for martingale problems, since those are solved on $\mathbb{R}^{n}$ and require uniform elliptic diffusion parts. Neither of these is satisfied in our case.

Now let $\left(\alpha, b, \beta^{i j}, c=0, \gamma=0, m, \mu\right)$ be some admissible parameter set. Fix some $\epsilon, \delta>0$ and $n \in \mathbb{N}$. In order to bound the coefficients and cut off the small jumps, we let

$$
\varphi_{n} \in C_{b}^{\infty}\left(S_{d}\right), \quad 0 \leq \varphi_{n} \leq 1, \quad \varphi_{n}(x)= \begin{cases}1, & \|x\| \leq n \\ \frac{n}{\|x\|}, & \|x\| \geq n+1 .\end{cases}
$$

We then define the bounded and smooth parameters

$$
\begin{aligned}
B^{n}(x) & =B\left(\varphi_{n}(x) x\right) \\
m^{\delta}(d \xi) & =m(d \xi) 1_{\{\|\xi\|>\delta\}} \\
M^{\delta, n}(x, d \xi) & =\left\langle\varphi_{n}(x) x, \frac{\mu(d \xi)}{\|\xi\|^{2} \wedge 1} 1_{\{\|\xi\|>\delta\}}\right\rangle .
\end{aligned}
$$

Concerning the diffusion function $A_{i j k l}(x)$ given by (2.13), we first find an appropriate factorization which will allow us to write the continuous martingale part of $X$ as a stochastic integral. Thereto observe that any $S_{d}^{+}$-valued solution, presumed that it exists, of the following symmetric matrix-valued diffusion SDE

$$
d Z_{t}=\sqrt{Z_{t}} d W_{t} \Sigma+\Sigma^{\top} d W_{t}^{\top} \sqrt{Z_{t}},
$$

where $W_{t}$ is a standard $d \times d$-matrix Brownian motion and $\Sigma \in M_{d}$ with $\Sigma^{\top} \Sigma=\alpha$, has quadratic variation $d\left\langle Z_{i j}, Z_{k l}\right\rangle_{t}=A_{i j k l}\left(Z_{t}\right)$. Define now $\sigma^{k l}(x) \in S_{d}$ by

$$
\sigma^{k l}(x)=\sqrt{x} M^{k l} \Sigma+\Sigma^{\top} M^{l k} \sqrt{x}
$$

where $M_{i j}^{k l}=\delta_{i k} \delta_{j l}$. Then (5.5) can be written as

$$
d Z_{t}=\sum_{k, l=1}^{d} \sigma^{k l}\left(Z_{t}\right) d W_{t, k l}
$$

and $A_{i j k l}(x)=\sum_{m, n=1}^{d} \sigma_{i j}^{m n}(x) \sigma_{k l}^{m n}(x)$.

Since $\sigma^{k l}(x)$ involves the matrix square root, which is neither Lipschitz continuous nor bounded nor globally defined, we need to introduce some approximating regularization in order to meet the assumptions of Theorem A.5. Thereto fix some 
truncation function

$$
\eta_{\varepsilon} \in C_{b}^{\infty}\left(S_{d}\right), \quad \eta_{\varepsilon}(x)= \begin{cases}1, & x \in S_{d}^{+} \\ 0, & x \notin S_{d}^{+}-\varepsilon I,\end{cases}
$$

and define

$$
s_{\varepsilon, n}(x)= \begin{cases}\eta_{\varepsilon}\left(\varphi_{n}(x) x\right)\left(\sqrt{\varphi_{n}(x) x+\varepsilon I_{d}}-\sqrt{\varepsilon I_{d}}\right), & \text { if } x \in S_{d}^{+}-\varepsilon I \\ 0, & \text { otherwise }\end{cases}
$$

Note that $s_{\varepsilon, n}$ satisfies

- $s_{\varepsilon, n} \in C_{b}^{\infty}\left(S_{d}, S_{d}\right)$,

- $s_{\varepsilon, n}(x)=\left(\sqrt{\varphi_{n}(x) x+\varepsilon I_{d}}-\sqrt{\varepsilon I_{d}}\right)$ on $S_{d}^{+}$,

- $\lim _{\varepsilon \rightarrow 0^{+}} s_{\varepsilon, n}(x)=\sqrt{\varphi_{n}(x) x}$.

With this we can now define the regularization of $\sigma^{k l}$ by

$$
\sigma_{\varepsilon, n}^{k l}(x)=s_{\varepsilon, n}(x) M^{k l} \Sigma+\Sigma^{\top} M^{l k} s_{\varepsilon, n}(x),
$$

which then satisfies the smoothness condition of Theorem A.5. Finally, we set

$$
\begin{aligned}
A_{i j k l}^{\varepsilon, n}(x) & =\sum_{m, n}^{d}\left(\sigma_{\varepsilon, n}^{m n}(x)\right)_{i j}\left(\sigma_{\varepsilon, n}^{m n}(x)\right)_{k l} \\
& =\left(s_{\varepsilon, n}^{2}(x)\right)_{i k} \alpha_{j l}+\left(s_{\varepsilon, n}^{2}(x)\right)_{i l} \alpha_{j k}+\left(s_{\varepsilon, n}^{2}(x)\right)_{j k} \alpha_{i l}+\left(s_{\varepsilon, n}^{2}(x)\right)_{j l} \alpha_{i k}
\end{aligned}
$$

and define the corresponding regularized operator on $C_{0}\left(S_{d}\right)$

$$
\begin{aligned}
\mathcal{A}^{\varepsilon, \delta, n} f(x)= & \frac{1}{2} \sum_{i, j, k, l} A_{i j k l}^{\varepsilon, n}(x) \frac{\partial^{2} f(x)}{\partial x_{i j} \partial x_{k l}}+\sum_{i, j}\left(b_{i j}+B_{i j}^{n}(x)\right) \frac{\partial f(x)}{\partial x_{i j}} \\
& +\int_{S_{d}^{+} \backslash\{0\}}(f(x+\xi)-f(x)) m^{\delta}(d \xi) \\
& +\int_{S_{d}^{+} \backslash\{0\}}(f(x+\xi)-f(x)-\langle\chi(\xi), \nabla f(x)\rangle) M^{\delta, n}(x, d \xi) .
\end{aligned}
$$

We now show that $\mathcal{A}^{\varepsilon, \delta, n}$ approximates $\mathcal{A}$. We let $\mathcal{S}=\mathcal{S}\left(S_{d}\right)$ and $\mathcal{S}_{+}$denote the locally convex spaces of rapidly decreasing $C^{\infty}$-functions on $S_{d}$ and $S_{d}^{+}$defined in (B.1) below, respectively.

Lemma 5.5. $\mathcal{S} \subset \mathcal{D}\left(\mathcal{A}^{\varepsilon, \delta, n}\right)$ and, for every $f \in \mathcal{S}_{+}$,

$$
\lim _{\varepsilon, \delta, n}\left\|\mathcal{A}^{\varepsilon, \delta, n} f-\mathcal{A} f\right\|_{\infty}=0
$$


Proof. Since $\varphi_{n}$ as defined in (5.4) converges uniformly on compact sets to 1 , this is clear for the differential operator part. Concerning the integral part, we have

$$
\begin{aligned}
& \left\|\int_{S_{d}^{+} \backslash\{0\}}\left(f(x+\xi)-f(x)-\left\langle 1_{\{\|\xi\| \leq 1\}} \xi, \nabla f(x)\right\rangle\right)\left(M^{\delta, n}(x, d \xi)-M(x, d \xi)\right)\right\| \leq \\
& \left\|\sum_{i, j} \int_{S_{d}^{+} \backslash\{0\}}\left(\frac{f(x+\xi)-f(x)-\left\langle 1_{\{\|\xi\| \leq 1\}} \xi, \nabla f(x)\right\rangle}{\|\xi\|^{2} \wedge 1}\right) x_{i j}\left(\varphi_{n}(x)-1\right) \mu_{i j}^{\delta}(d \xi)\right\|+ \\
& \left\|\sum_{i, j} \int_{S_{d}^{+} \backslash\{0\}}\left(\frac{f(x+\xi)-f(x)-\left\langle 1_{\{\|\xi\| \leq 1\}} \xi, \nabla f(x)\right\rangle}{\|\xi\|^{2} \wedge 1}\right) x_{i j}\left(1_{\{\|\xi\|>\delta\}}-1\right) \mu_{i j}(d \xi)\right\| .
\end{aligned}
$$

By dominated convergence the second term goes uniformly in $x$ to 0 , thus we only have to consider the first one. By splitting the first integral into $\int_{\{\|\xi\| \leq 1\}}+\int_{\{\|\xi\|>1\}}$, we note that $\left\|\int_{\{\|\xi\| \leq 1\}}\right\|$ converges uniformly in $x$ to 0 . Hence it remains to analyze

$$
\left\|\sum_{i, j} \int_{\{\|\xi\|>1\}}(f(x+\xi)-f(x)) x_{i j}\left(\varphi_{n}(x)-1\right) \mu_{i j}(d \xi)\right\|,
$$

which can be estimated by

$$
\begin{aligned}
& \sum_{i, j}\left(\int_{\{\|\xi\|>1\}}\left\|f(x+\xi) x_{i j}\left(\varphi_{n}(x)-1\right)\right\|\left(\mu_{i j}^{+}(d \xi)+\mu_{i j}^{-}(d \xi)\right)\right. \\
& \left.\quad+\int_{\{\|\xi\|>1\}}\left\|f(x) x_{i j}\left(\varphi_{n}(x)-1\right)\right\|\left(\mu_{i j}^{+}(d \xi)+\mu_{i j}^{-}(d \xi)\right)\right)
\end{aligned}
$$

where $\mu_{i j}^{+}, \mu_{i j}^{-}$correspond to the Jordan decomposition of $\mu_{i j}=\mu_{i j}^{+}-\mu_{i j}^{-}$. As $f$ lies in $\mathcal{S}_{+}$, the second term converges uniformly to 0 . For the first one, observe that for every $n$

$$
\left\|f(x+\xi) x_{i j}\left(\varphi_{n}(x)-1\right)\right\| \leq\left\|f(x+\xi) x_{i j}\right\| \leq\|f(x+\xi)\| x+\xi\|\|,
$$

such that we can apply dominated convergence. Again, since $f$ lies in $\mathcal{S}_{+}$, the first integral converges uniformly in $x$ to 0 as well. Hence (5.11) holds true, and $\mathcal{S} \subset \mathcal{D}\left(\mathcal{A}^{\varepsilon, \delta, n}\right)$ follows similarly.

We now establish existence for the martingale problem for $\mathcal{A}^{\varepsilon, \delta, n}$.

Lemma 5.6. For every $x \in S_{d}^{+}$there exists an $S_{d}^{+}$-valued càdlàg solution $X$ to the martingale problem for $\mathcal{A}^{\varepsilon, \delta, n}$ with $X_{0}=x$. That is

$$
f\left(X_{t}\right)-\int_{0}^{t} \mathcal{A}^{\varepsilon, \delta, n} f\left(X_{s}\right) d s
$$

is a martingale, for all $f \in \mathcal{S}$.

Proof. Consider the following SDE of type A.1

$$
\begin{aligned}
X_{t}^{\varepsilon, \delta, n}= & x+\int_{0}^{t}\left(b+B^{n}\left(X_{s}^{\varepsilon, \delta, n}\right)-\int_{S_{d}^{+} \backslash\{0\}} \chi(\xi) M^{\delta, n}\left(X_{s}^{\varepsilon, \delta, n}, d \xi\right)\right) d s \\
& +\sum_{k, l}^{d} \int_{0}^{t} \sigma_{\varepsilon, n}^{k l}\left(X_{s}^{\varepsilon, \delta, n}\right) d W_{s, k l}+J_{t},
\end{aligned}
$$


where $W_{t}$ is a $d \times d$-matrix of standard Brownian motions and $J_{t}$ a finite activity jump process with compensator $m^{\delta}(d \xi)+M^{\delta, n}\left(X_{t}^{\varepsilon, \delta, n}, d \xi\right)$. Note that the quadratic variation of the continuous martingale part of $X_{t}^{\varepsilon, \delta, n}$ is given by $A_{i j k l}^{\varepsilon, n}(x)$ as defined in (5.9). It thus follows by inspection that any càdlàg solution $X^{\varepsilon, \delta, n}$ of (5.12) solves the martingale problem for $\mathcal{A}^{\varepsilon, \delta, n}$.

Hence it remains to show that there exists an $S_{d}^{+}$-valued càdlàg solution of (5.12). Let us recall the normal cone (2.23) to $S_{d}^{+}$. As $b+B^{n}(x)-\int_{S_{d}^{+} \backslash\{0\}} \chi(\xi) M^{\delta, n}(x, d \xi)$, $\sigma_{\varepsilon, n}^{k l}(x)$ and $m^{\delta}(d \xi)+M^{\delta, n}(x, d \xi)$ are designed to satisfy the assumptions of Theorem A.5 and since $x+\operatorname{supp}\left(m^{\delta}(\cdot)+M^{\delta, n}(x, \cdot)\right) \subseteq S_{d}^{+}$for all $x \in S_{d}^{+}$, we only have to show that for all $x \in \partial S_{d}^{+}$and $u \in N_{S_{d}^{+}}(x)$

$$
\begin{aligned}
\left\langle\sigma_{\varepsilon, n}^{k l}(x), u\right\rangle & =0, \\
\left\langle b+B^{n}(x)-\int_{S_{d}^{+} \backslash\{0\}} \chi(\xi) M^{\delta, n}(x, d \xi)-\frac{1}{2} \sum_{k, l=1}^{d} D \sigma_{\varepsilon, n}^{k l}(x) \sigma_{\varepsilon, n}^{k l}(x), u\right\rangle & \geq 0 .
\end{aligned}
$$

Due to the definition of $\sigma_{\varepsilon, n}^{k l}(x)$, respectively the definition of $s_{\varepsilon, n}(x)$ given in (5.7), condition (5.13) is satisfied. Concerning (5.14), we have by (2.11)

$$
\left\langle B^{n}(x)-\int_{S_{d}^{+} \backslash\{0\}} \chi(\xi) M^{\delta, n}(x, d \xi), u\right\rangle \geq 0 .
$$

Moreover, it is shown in Lemma 5.7 below that

$$
\left\langle b-\frac{1}{2} \sum_{k, l=1}^{d} D \sigma_{\varepsilon, n}^{k l}(x) \sigma_{\varepsilon, n}^{k l}(x), u\right\rangle \geq 0 .
$$

Whence the lemma now follows from Theorem A.5.

Lemma 5.7. Let $x=O \Lambda O^{\top} \in S_{d}^{+}$where $\Lambda=\operatorname{diag}\left(\lambda_{1}, \ldots, \lambda_{d}\right)$ contains the eigenvalues in decreasing order and let $\sigma_{\varepsilon, n}^{k l}$ be defined by (5.8). Then, for all $x \in S_{d}^{+}$,

$$
\begin{aligned}
\frac{1}{2} \sum_{k, l=1}^{d} D \sigma_{\varepsilon, n}^{k l}(x) \sigma_{\varepsilon, n}^{k l}(x)= & \frac{1}{2} \sum_{i=1}^{d} \frac{\varphi_{n}(x)\left(\sqrt{\lambda_{i} \varphi_{n}(x)+\varepsilon}-\sqrt{\varepsilon}\right)}{\sqrt{\lambda_{i} \varphi_{n}(x)+\varepsilon}} U^{i} \\
& +\frac{1}{2} \sum_{i \neq j} \frac{\varphi_{n}(x)\left(\sqrt{\lambda_{j} \varphi_{n}(x)+\varepsilon}-\sqrt{\varepsilon}\right)}{\sqrt{\lambda_{i} \varphi_{n}(x)+\varepsilon}+\sqrt{\lambda_{j} \varphi_{n}(x)+\varepsilon}} U^{i} \\
& +\frac{1}{2} \sum_{i, k, l} \frac{\lambda_{i}}{2 \sqrt{\lambda_{i} \varphi_{n}(x)+\varepsilon}}\left\langle\nabla \varphi_{n}(x), \sigma_{\varepsilon, n}^{k l}\right\rangle Z^{i k l},
\end{aligned}
$$

where $U_{m n}^{i}=\left(\left(\Sigma^{\top} \Sigma\right) O\right)_{m i} O_{n i}+\left(\left(\Sigma^{\top} \Sigma\right) O\right)_{n i} O_{m i}$ and $Z_{m n}^{i k l}=O_{m i} O_{k i} \Sigma_{l n}+O_{n i} O_{k i} \Sigma_{l m}$.

Furthermore, if

$$
b \succeq(d-1) \Sigma^{\top} \Sigma,
$$

then

$$
\left\langle b-\frac{1}{2} \sum_{k, l=1}^{d} D \sigma_{\varepsilon, n}^{k l}(x) \sigma_{\varepsilon, n}^{k l}(x), u\right\rangle \geq 0
$$

for all $x \in \partial S_{d}^{+}$and for all $u \in N_{S_{d}^{+}}(x)$. 
Proof. Let us denote

$$
C^{\varepsilon, n}(x)=\frac{1}{2} \sum_{k, l=1}^{d} D \sigma_{\varepsilon, n}^{k l}(x) \sigma_{\varepsilon, n}^{k l}(x)
$$

and notice that

$$
C^{\varepsilon, n}(x)=\frac{1}{2} \sum_{k, l}\left(\left.\frac{d}{d t} s_{\varepsilon, n}\left(x+t \sigma_{\varepsilon, n}^{k l}(x)\right)\right|_{t=0} M^{k l} I_{r}^{d}+\left.I_{r}^{d}\left(M^{k l}\right)^{\top} \frac{d}{d t} s_{\varepsilon, n}\left(x+t \sigma_{\varepsilon, n}^{k l}(x)\right)\right|_{t=0}\right) .
$$

We now use the following formula from [30, Theorem 6.6.30]:

$$
\frac{d}{d t} f(V(t))=O(t)\left(\sum_{i, j} \Delta f\left(\lambda_{i}(t), \lambda_{j}(t)\right) M^{i i}\left[O(t)^{\top} V^{\prime}(t) O(t)\right] M^{j j}\right) O(t)^{\top},
$$

where $V(t)=O(t) \operatorname{diag}\left(\lambda_{1}(t), \ldots, \lambda_{d}(t)\right) O(t)^{\top}$ is a family of symmetric matrices and $\Delta f(u, v)=\frac{(f(u)-f(v))}{(u-v)}$ for $u \neq v$ and $\Delta f(u, u)=f^{\prime}(u)$. This holds true if $V(\cdot)$ is continuously differentiable for $t \in(a, b)$ and $f(\cdot)$ is continuously differentiable on an open real interval which contains all eigenvalues of $V(t)$ for all $t \in(a, b)$.

We now apply this formula to our case where $f(t)=\sqrt{t}$ and

$$
V(t)=\varphi_{n}\left(x+t \sigma_{\varepsilon, n}^{k l}\right)\left(x+t \sigma_{\varepsilon, n}^{k l}\right)+\varepsilon I_{d} .
$$

Since we take the derivative at $t=0$, we only have to consider

$$
V(0)=O\left(\varphi_{n}(x) \Lambda+\varepsilon I_{d}\right) O^{\top},
$$

where $O$ is the orthogonal matrix diagonalizing $x$ and

$$
V^{\prime}(0)=\left\langle\nabla \varphi_{n}(x), \sigma_{\varepsilon, n}^{k l}\right\rangle x+\varphi_{n}(x) \sigma_{\varepsilon, n}^{k l} .
$$

Note that we do not have an explicit contribution of $\eta_{\varepsilon}$ which is part of the definition of $s_{\varepsilon, n}$, since $\eta_{\varepsilon}\left(S_{d}^{+}\right)=1$ and $\nabla \eta_{\varepsilon}\left(S_{d}^{+}\right)=0$. Some lines of calculations then yield (5.15).

Let us now verify (5.17). Take an arbitrary $x=O \Lambda O^{\top} \in \partial S_{d}^{+}$and assume first that it has rank $d-1$, that is $\lambda_{d}=0$ and all other eigenvalues are strictly positive. By Lemma 4.1 and (2.23) the elements of $N_{S_{d}^{+}}(x)$ can then be written as $u=O K O^{\top}$ where $K=\operatorname{diag}(0, \ldots, 0, k)$ with $k \geq 0$. Thus, (5.17) now reads

$$
\left\langle b-C^{\varepsilon, n}(x), O K O^{\top}\right\rangle=k\left[O^{\top} b O-O^{\top} C^{\varepsilon, n}(x) O\right]_{d d} .
$$

As $\left[O^{\top} U^{i} O\right]_{d d}=2 \delta_{i d}\left(O^{\top} \Sigma^{\top} \Sigma O\right)_{i d}$ and $O^{\top} Z^{i k l} O=2 \delta_{i d} O_{k i}(\Sigma O)_{l d}$, we have

$$
\left[O^{\top} C^{\varepsilon, n}(x) O\right]_{d d}=\sum_{j \neq d} \frac{\varphi_{n}(x)\left(\sqrt{\lambda_{j} \varphi_{n}(x)+\varepsilon}-\sqrt{\varepsilon}\right)}{\sqrt{\lambda_{j} \varphi_{n}(x)+\varepsilon}+\sqrt{\varepsilon}}\left[O^{\top} \Sigma^{\top} \Sigma O\right]_{d d} .
$$

Since $\sum_{j \neq d} \frac{\varphi_{n}(x)\left(\sqrt{\lambda_{j} \varphi_{n}(x)+\varepsilon}-\sqrt{\varepsilon}\right)}{\sqrt{\lambda_{j} \varphi_{n}(x)+\varepsilon}+\sqrt{\varepsilon}} \leq d-1$, we obtain by condition (5.16)

$$
\left[O^{\top} b O-O^{\top} C^{\varepsilon, n}(x) O\right]_{d d} \geq\left[O^{\top}\left(b-(d-1) \Sigma^{\top} \Sigma\right) O\right]_{d d} \geq 0
$$

which proves (5.17) for $x \in \partial S_{d}^{+}$with $\mathrm{rk}=d-1$. In the general case we can proceed similarly. For $x \in \partial S_{d}^{+}$with $\mathrm{rk}=r \leq d-1$, the elements of $N_{S_{d}^{+}}(x)$ are given by $u=O K O^{\top}$ where

$$
K=\left(\begin{array}{ll}
0 & 0 \\
0 & k
\end{array}\right)
$$


with $k \in S_{d-r}^{+}$. This follows again from Lemma 4.1 and (2.23). Now, (5.17) can be written as

$$
\begin{aligned}
\langle b & \left.-C^{\varepsilon, n}(x), O K O^{\top}\right\rangle \\
& =\left\langle O^{\top}\left(b-\sum_{j \leq r} \frac{\varphi_{n}(x)\left(\sqrt{\lambda_{j} \varphi_{n}(x)+\varepsilon}-\sqrt{\varepsilon}\right)}{\sqrt{\lambda_{j} \varphi_{n}(x)+\varepsilon}+\sqrt{\varepsilon}} \Sigma^{\top} \Sigma\right) O, K\right\rangle \\
& \geq\left\langle O^{\top}\left(b-r \Sigma^{\top} \Sigma\right) O, K\right\rangle \geq 0
\end{aligned}
$$

which proves the assertion.

Combining Lemmas 5.5 and [5.6, we obtain the announced existence result for the martingale problem for $\mathcal{A}$ :

Lemma 5.8. For every $x \in S_{d}^{+}$there exists an $S_{d}^{+} \cup\{\Delta\}$-valued càdlàg solution $X$ to the martingale problem for $\mathcal{A}$ with $X_{0}=x$. That is

$$
f\left(X_{t}\right)-\int_{0}^{t} \mathcal{A} f\left(X_{s}\right) d s
$$

is a martingale, for all $f \in \mathcal{S}_{+}$.

Proof. By Lemma [5.6] there exists a solution $X^{\varepsilon, \delta, n}$ to the martingale problem for $\mathcal{A}^{\varepsilon, \delta, n}$ with sample paths in $\mathbb{D}\left(S_{d}^{+}\right)$and hence also in $\mathbb{D}\left(S_{d}^{+} \cup\{\Delta\}\right)$. We now claim that $\left(X^{\varepsilon, \delta, n}\right)$ is relatively compact considered as a sequence of processes with sample paths in $\mathbb{D}\left(S_{d}^{+} \cup\{\Delta\}\right)$. For the proof of this assertion we shall make use of Theorems 9.1 and 9.4 in Chapter 3 of [18. In order to meet the assumption of [18, Chap. 3, Theorem 9.4], we take $C_{c}^{\infty}\left(S_{d}^{+}\right)$as subalgebra of $C_{b}\left(S_{d}^{+}\right)$. Then, for every $T>0$ and $f \in C_{c}^{\infty}\left(S_{d}^{+}\right)$, we have

$$
\sup _{\varepsilon, \delta, n} \mathbb{E}\left[\operatorname{essup}_{t \in[0, T]}\left|\mathcal{A}^{\varepsilon, \delta, n} f\left(X_{t}^{\varepsilon, \delta, n}\right)\right|\right]<\infty,
$$

since there exists a constant $C$ such that $\left\|\mathcal{A}^{\varepsilon, \delta, n} f\right\|_{\infty} \leq C p_{3,+}(f)<\infty$ for all $n$, where $p_{k,+}$ are the semi-norms as defined in (B.2). Thus, the requirements of [18, Chap. 3, Theorem 9.4] are satisfied. Note that $Y$ in the notation of [18, Chap. 3, Theorem 9.4] corresponds in our case to $f(X)$ such that [18, Chap. 3, Condition (9.17)] is automatically fulfilled. It then follows by the conclusion of [18, Chap. 3, Theorem 9.4] that $\left(f\left(X_{t}^{\varepsilon, \delta, n}\right)\right)$ is relatively compact (as family of process with sample paths in $\mathbb{R}$ ) for each $f \in C_{c}^{\infty}$. Furthermore, since we consider $S_{d}^{+} \cup\{\Delta\}$, the compact containment condition is always satisfied, that is for every $\eta>0$ and $T>0$, there exists a compact set $\Gamma_{\eta, T} \subset\left(S_{d}^{+} \cup\{\Delta\}\right)$ for which

$$
\inf _{\varepsilon, \delta, n} \mathbb{P}\left[X_{t}^{\varepsilon, \delta, n} \in \Gamma_{\varepsilon, T} \text { for } t \in[0, T]\right] \geq 1-\eta
$$

holds true. By [18, Chap. 3, Theorem 9.1] and the fact that $\left\{1, C_{c}^{\infty}\left(S_{d}^{+}\right)\right\}$is dense in $C\left(S_{d}^{+} \cup\{\Delta\}\right)$, we therefore obtain that $\left(X^{\varepsilon, \delta, n}\right)$ is relatively compact in $\mathbb{D}\left(S_{d}^{+} \cup\{\Delta\}\right)$. Thus, by [18, Chap. 3, Theorem 3.1] there exists a subsequence $X^{\varepsilon_{k}, \delta_{k}, n_{k}}$ which converges in distribution to some limit process $X$ in $\mathbb{D}\left(S_{d}^{+} \cup\{\Delta\}\right)$.

Combining this with Lemma 5.5 and [18, Chap. 4, Lemma 5.1], we conclude that $X$ is a solution to the martingale problem for $\mathcal{A}$. Hence the lemma is proved. 
We can now prove the existence and uniqueness of an affine process for any admissible parameter set.

Proposition 5.9. Let $\left(\alpha, b, \beta^{i j}, c, \gamma, m, \mu\right)$ be an admissible parameter set. Then there exists a unique affine process on $S_{d}^{+}$with infinitesimal generator (2.12), and (2.1) holds for all $(t, u) \in \mathbb{R}_{+} \times S_{d}^{+}$, where $\phi(t, u)$ and $\psi(t, u)$ are given by (2.14) and (2.15).

Proof. Suppose first that $c=0$ and $\gamma=0$. Let $x_{0} \in S_{d}^{+}$. Then Lemma 5.8 implies the existence of an $S_{d}^{+} \cup\{\Delta\}$-valued càdlàg solution $X$ of the martingale problem for $\mathcal{A}$ with $X_{0}=x_{0}$. We now show that $X$ is unique in distribution.

Thereto, note that by [18, Chap. 4, Theorem 7.1],

$$
f\left(t, X_{t}\right)-\int_{0}^{t}\left(\mathcal{A} f\left(s, X_{s}\right)+\partial_{s} f\left(s, X_{s}\right)\right) d s
$$

is a martingale for all rapidly decreasing functions $f \in \mathcal{S}\left(\mathbb{R}_{+} \times S_{d}^{+}\right)$, similarly defined as $\mathcal{S}_{+}$in (B.1). Now let $\phi$ and $\psi$ be the unique solutions of the generalized Riccati differential equations (2.14) - 2.15), given by Proposition 5.3. Fix $t>0, u \in S_{d}^{++}$, and some $f \in \mathcal{S}\left(\mathbb{R}_{+} \times S_{d}^{+}\right)$such that

$$
f(s, x)=e^{-\phi(t-s, u)-\langle\psi(t-s, u), x\rangle}, \quad 0 \leq s \leq t, x \in S_{d}^{+} .
$$

Then

$$
\mathcal{A} f(s, x)+\partial_{s} f(s, x)=0, \quad 0 \leq s \leq t, x \in S_{d}^{+} .
$$

In view of (5.18), the Laplace transform of $X_{t}$ at $u$ is thus given by

$$
\mathbb{E}\left[e^{-\left\langle u, X_{t}\right\rangle}\right]=\mathbb{E}\left[f\left(t, X_{t}\right)\right]=f\left(0, x_{0}\right)-0=e^{-\phi(t, u)-\left\langle\psi(t, u), x_{0}\right\rangle} .
$$

Since $u \in S_{d}^{++}$was arbitrary, we conclude that the distribution of $X_{t}$ is uniquely determined for all $t>0$. From [18, Chap. 4, Theorem 4.1] we infer that $X$ is a Markov process with generator $\mathcal{A}$ on $\mathcal{S}_{+}$and thus unique in law as solution of the martingale problem for $\mathcal{A}$. Moreover, by (5.19), $X$ is stochastically continuous and affine. Thus the proposition is proved under the premise that $c=0$ and $\gamma=0$.

For general parameters $c$ and $\gamma$ we employ a Feynman-Kac argument. Denote by $\mathcal{B}$ and $\left(Q_{t}\right)$ the affine generator and corresponding Feller semigroup associated with $\left(\alpha, b, \beta^{i j}, c=0, \gamma=0, m, \mu\right)$ from the first part of the proof, respectively. Since $x \mapsto c+\langle\gamma, x\rangle$ is nonnegative on $S_{d}^{+}$, it follows along the lines of [17, Proposition 11.1], that

$$
P_{t} f(x)=\mathbb{E}_{x}\left[e^{-\int_{0}^{t} c+\left\langle\gamma, X_{s}\right\rangle d s} f\left(X_{t}\right)\right]
$$

defines a Feller semigroup $\left(P_{t}\right)$ on $C_{0}\left(S_{d}^{+}\right)$with infinitesimal generator $\mathcal{A}(x)=$ $\mathcal{B} f(x)-(c+\langle\gamma, x\rangle) f(x)$ for $f \in C_{c}^{\infty}\left(S_{d}^{+}\right)$, which is the desired solution.

5.3. An Alternative Existence Proof for Jump Processes. For affine processes without diffusion component (that is the admissible parameter $\alpha$ vanishes), the existence question can be handled entirely as in the case of affine processes on $\mathbb{R}_{+}^{m} \times \mathbb{R}^{n}$ [17, Section 7]. In this section we elaborate an alternative existence proof in this specific case, by following the lines of [17. Note that the OU-type processes driven by matrix Lévy subordinators 4 are contained in the class of pure jump processes of this section. 
We call a function $f: S_{d}^{+} \rightarrow \mathbb{R}$ of Lévy-Khintchine form on $S_{d}^{+}$, if

$$
f(u)=\left\langle b_{0}, u\right\rangle-\int_{S_{d}^{+} \backslash\{0\}}\left(e^{-\langle u, \xi\rangle}-1\right) m_{0}(d \xi),
$$

where $b_{0} \in S_{d}^{+}$and $m_{0}$ is a Borel measure supported on $S_{d}^{+}$such that

$$
\int_{S_{d}^{+} \backslash\{0\}}(\|\xi\| \wedge 1) m_{0}(d \xi)<\infty .
$$

Once again, we recall that a distribution on $S_{d}^{+}$is infinitely divisible if and only if its Laplace transform takes the form $e^{-f(u)}$, where $f$ is of the above form (see also Step 1 in the proof of Proposition 4.9).

Similarly to [17, we introduce the sets

$$
\begin{aligned}
\mathcal{C} & :=\left\{f+c \mid f: S_{d}^{+} \rightarrow \mathbb{R} \text { is of Lévy-Khintchine form on } S_{d}^{+}, c \in \mathbb{R}_{+}\right\}, \\
\mathcal{C}_{S} & :=\left\{\psi \mid u \mapsto\langle\psi(u), w\rangle \in \mathcal{C} \text { for all } w \in S_{d}^{+}\right\} .
\end{aligned}
$$

The following technical statement can be obtained easily by mimicking the proofs of the corresponding statements in [17, Proposition 7.2 and 7.4 and Lemma 7.5]:

Lemma 5.10. We have,

(i) $\mathcal{C}, \mathcal{C}_{S}$ are convex cones in $C\left(S_{d}^{+}\right)$.

(ii) $\phi \in \mathcal{C}, \psi \in \mathcal{C}_{S}$ imply $\phi(\psi) \in \mathcal{C}$.

(iii) $\psi, \psi_{1} \in \mathcal{C}_{S}$ imply $\psi_{1}(\psi) \in \mathcal{C}_{S}$.

(iv) If $\phi_{k} \in \mathcal{C}$ converges to a continuous function $\phi$ on $S_{d}^{+}$, then $\phi \in \mathcal{C} . A$ similar statement holds for sequences in $\mathcal{C}_{S}$.

(v) Let $\left(\alpha=0, b, \beta^{i j}, c, \gamma, m, \mu\right)$ be an admissible parameter set. If $\mu=\left(\mu_{i j}\right)_{i j}$ satisfy the integrability condition

$$
\int_{S_{d}^{+} \backslash\{0\}} \frac{\mu_{i j}(d \xi)}{\|\xi\| \wedge 1}<\infty
$$

for all $i \leq j$, then for $F$ and $R$, as defined in (2.16) and (2.17), we have $F \in \mathcal{C}$ and $R \in \mathcal{C}_{S}$.

(vi) Let $\left(\alpha=0, b, \beta^{i j}, c, \gamma, m, \mu\right)$ be an admissible parameter set. There exists a sequence of functions $\left(g_{k}\right)_{k \in \mathbb{N}}$ of the form (2.17) which converge locally uniformly to $R$ such that the linear jump coefficients $\mu_{k}$ in $g_{k}$ satisfies (5.20) for every $k \in \mathbb{N}$. In particular $R_{\delta} \rightarrow R$ locally uniformly as $\delta \rightarrow 0$, where $R_{\delta}$ corresponds to the admissible parameter set $\left(\alpha=0, b, \beta^{i j}, c, \gamma, m, \mu 1_{\{\|\xi\| \geq \delta\}}\right)$.

Remark 5.11. We note that the proof of statement (vi) is much easier than the one of [17, Lemma 7.5], because $\alpha=0$. However, for $\alpha \neq 0, R$ cannot be approximated in Lévy-Khintchine form anyway: Otherwise one could infer (by means of the proof of the subsequent statement) the existence of an affine process which is infinitely decomposable and has non-vanishing diffusion component. This is in contradiction with Proposition 2.10 and in the case of pure diffusion it contradicts Example 2.9.

Proposition 5.12. For all $t \geq 0$, the solutions $(\phi(t, \cdot), \psi(t, \cdot))$ of (2.14) and (2.15) lie in $\left(\mathcal{C}, \mathcal{C}_{S}\right)$. 
Proof. Suppose first, (5.20) is satisfied. Then equation (2.15) is equivalent to the integral equation

$$
\psi(t, u)=e^{\widetilde{B} t}(u)+\int_{0}^{t} e^{\widetilde{B}(t-s)}(\widetilde{R}(\psi(s, u)) d s,
$$

where we have set $R(u)=\widetilde{R}(u)+\widetilde{B}^{\top}(u)$ and $\widetilde{B}^{\top} \in \mathcal{L}\left(S_{d}\right)$ is given by

$$
\widetilde{B}^{\top}(u):=B^{\top}(u)-\int_{S_{d}^{+} \backslash\{0\} \cap\|\xi\| \leq 1} \frac{\langle\chi(\xi), u\rangle}{\|\xi\|^{2} \wedge 1} \mu(d \xi) .
$$

and $e^{\widetilde{B} t}(u)$ is the notation for the semi-group induced by $\partial_{t} x(t, u)=\widetilde{B}^{\top}(x(t, u))$, $x(0, u)=u$. Hence, the variation of constants formula yields (5.21).

Due to admissibility condition (2.11), we have that $\widetilde{B}^{\top}$ is a linear drift which is "inward pointing" at the boundary of $S_{d}^{+}$, which is equivalent to $e^{\widetilde{B} t}$ being a positive semi-group, that is, $e^{\widetilde{B} t}$ maps $S_{d}^{+}$into $S_{d}^{+}$. Also, we have by construction that $\widetilde{R} \in \mathcal{C}_{S}$.

Using Picard iteration and Lemma 5.10 , it follows that the sequence $\psi^{(k)}$ defined as

$$
\begin{aligned}
\psi^{(0)}(t, u) & :=u, \\
\psi^{(k+1)}(t, u) & :=e^{\widetilde{B} t}(u)+\int_{0}^{t} e^{\widetilde{B}(t-s)}\left(\widetilde{R}\left(\psi^{(k)}(s, u)\right) d s\right.
\end{aligned}
$$

lies in $\mathcal{C}_{S}$, for each $t \geq 0$, hence so does its limit $\psi(t, \cdot)$ and $\phi(t, \cdot)=\int_{0}^{t} F(\psi(s, \cdot)) d s \in$ $\mathcal{C}$.

The general case is then reduced to the former, by an application of Lemma 5.10 (vi)

We are prepared to provide an alternative proof of Proposition 5.9 under the additional assumption $\alpha=0$ : By Proposition [5.12, $(\phi(t, \cdot), \psi(t, \cdot))$ lie in $\left(\mathcal{C}, \mathcal{C}_{S}\right)$. Hence for all $t \geq 0, x \in S_{d}^{+}$, there exists a sub-stochastic kernel $p_{t}(x, d \xi)$ with Laplace-transform $e^{-\phi(t, u)-\langle\psi(t, u), x\rangle}$. The Chapman-Kolmogorov equations hold in view of properties (3.1) and (3.2). Whence Proposition 5.9 follows.

\section{Proof of the Main Results}

6.1. Proof of Theorem 2.4. The first part is a summary of Propositions 3.4, 4.9 4.11 and 4.17. The second part follows from Proposition 5.9.

6.2. Proof of Theorem 2.5, Let $X$ be an affine process on $S_{d}^{+}$. We first derive two lemmas which assert that the affine property of the Laplace transform (2.1) carries over to the Fourier-Laplace transform of $X(t)$.

Lemma 6.1. The function

$$
P_{t} e^{-\langle u+\mathrm{i} v, x\rangle}=\int_{S_{d}^{+}} e^{-\langle u+\mathrm{i} v, \xi\rangle} p_{t}(x, d \xi)
$$

is analytic in $(t, u, v) \in \mathbb{R}_{+} \times S_{d}^{++} \times S_{d}$ and continuous in $(t, u, v) \in \mathbb{R}_{+} \times S_{d}^{+} \times S_{d}$. 
Proof. The continuity in $(t, u, v) \in \mathbb{R}_{+} \times S_{d}^{+} \times S_{d}$ follows as in Lemma 3.2(iii) above.

From Proposition 5.3 we know that $\phi(t, u)$ and $\psi(t, u)$, and hence $P_{t} e^{-\langle u, x\rangle}$, are analytic in $(t, u) \in \mathbb{R}_{+} \times S_{d}^{++}$. Moreover, by well-known regularity properties of Laplace transforms (see e.g. [20, Lemma 10.8]), $P_{t} e^{-\langle u, x\rangle}$ admits an analytic extension on the strip $S_{d}^{++}+\mathrm{i} S_{d}$ for every $t$. Let now $z_{0}=u_{0}+\mathrm{i} v_{0}$ with $u_{0} \in S_{d}^{++}$ and $v_{0} \in S_{d}$ and take some $v \in S_{d}$ such that $|v| \prec u_{0}$, where $|v|$ denotes the matrix where the eigenvalues of $v$ are replaced by their absolute values. Then for every $N \in \mathbb{N}$

$$
\begin{aligned}
\left.\sum_{n=0}^{N}\left(\sum_{i, j}^{d} \mathrm{i} v_{i j} \frac{\partial}{\partial u_{i j}}\right)^{n}\left(\int_{S_{d}^{+}} e^{-\left\langle u+\mathrm{i} v_{0}, \xi\right\rangle} p_{t}(x, d \xi)\right)\right|_{u=u_{0}} \\
=\sum_{n=0}^{N} \int_{S_{d}^{+}} e^{-\left\langle u_{0}+\mathrm{i} v_{0}, \xi\right\rangle} \frac{\langle v, \xi\rangle^{n}(-\mathrm{i})^{n}}{n !} p_{t}(x, d \xi)=: f_{N}\left(t, u_{0}, v_{0}, v\right)
\end{aligned}
$$

is analytic in $u_{0}, v_{0}, v, t$, if $P_{t} e^{-\left\langle u_{0}+\mathrm{i} v_{0}, x\right\rangle}$ is analytic in $u_{0}, v_{0}, t$. This follows from the fact that for every $N, f_{N}$ is a finite sum of analytic functions. By dominated convergence - since we assumed $|v| \prec u_{0}$ - the limit of $f_{N}(t)$ for $N \rightarrow \infty$ is given by

$$
\int_{S_{d}^{+}} e^{-\left\langle u_{0}+\mathrm{i}\left(v_{0}+v\right), \xi\right\rangle} p_{t}(x, d \xi) .
$$

As $f_{N}$ is locally uniformly bounded in all variables, Vitali's theorem (41, Chapter 1 , Proposition 7]) then implies that the limit is also analytic in $t$. Assume now that $z_{0}$ is a point where the local dependence on $u_{0}, v_{0}, t$ is analytic. Then the previous argument shows that we have in fact the same assertion for $z_{0}+\mathrm{i} v$ as long as $|v| \prec u_{0}$. As we have real analyticity in $u$ and $t$ for $(u, t) \in S_{d}^{++} \times \mathbb{R}_{+}$and as for any given $v$ there exists a constant $k$ such that $|v / k| \prec u_{0}$, we conclude real analyticity in all three variables for $z_{0}+\mathrm{i} S_{d}$.

Lemma 6.2. There exist a unique continuous $\left(\mathbb{R}_{+}+\mathrm{i} \mathbb{R}\right)$ - and $\left(S_{d}^{+}+\mathrm{i} S_{d}\right)$-valued extension of $\phi$ and $\psi$ to $\mathbb{R}_{+} \times\left(S_{d}^{+}+\mathrm{i} S_{d}\right)$, respectively, such that

$$
P_{t} e^{-\langle u+\mathrm{i} v, x\rangle}=\int_{S_{d}^{+}} e^{-\langle u+\mathrm{i} v, \xi\rangle} p_{t}(x, d \xi)=e^{-\phi(t, u+\mathrm{i} v)-\langle\psi(t, u+\mathrm{i} v), x\rangle}
$$

holds for all $(t, u, v) \in \mathbb{R}^{+} \times S_{d}^{+} \times S_{d}$ and $x \in S_{d}^{+}$. Moreover, $\phi: \mathbb{R}_{+} \times\left(S_{d}^{++}+\mathrm{i} S_{d}\right) \rightarrow$ $\mathbb{R}_{+}+\mathrm{i} \mathbb{R}$ and $\psi: \mathbb{R}_{+} \times\left(S_{d}^{++}+\mathrm{i} S_{d}\right) \rightarrow S_{d}^{++}+\mathrm{i} S_{d}$ are analytic.

Proof. Define $\Upsilon(t, z):=P_{t} e^{-\langle z, 0\rangle}$ for $z \in S_{d}^{+}+\mathrm{i} S_{d}$. Since, by Lemma6.1, $P_{t} e^{-\langle u+\mathrm{i} v, x\rangle}$ is analytic in $(u, v) \in S_{d}^{++} \times S_{d}$ and since

$$
P_{t} e^{-\langle u, x\rangle} P_{t} e^{-\langle u, y\rangle}=P_{t} e^{-\langle u, x+y\rangle} \Upsilon(t, u)
$$

holds for all $u, x, y \in S_{d}^{+}$, this equality extends in $u$ to $S_{d}^{++}+\mathrm{i} S_{d}$. Thus, on the following set

$$
D=\left\{(t, z) \in \mathbb{R}_{+} \times\left(S_{d}^{++}+\mathrm{i} S_{d}\right) \mid \Upsilon(t, z) \neq 0\right\}
$$

the function

$$
g(t, z, x)=\frac{P_{t} e^{-\langle z, x\rangle}}{\Upsilon(t, z)}
$$

is well defined and satisfies the Cauchy functional equation

$$
g(t, z, x+y)=g(t, z, x) g(t, z, y) .
$$


By the continuity of $x \mapsto g(t, z, x)$ and the fact that $g(t, z, 0)=1$ for all $(t, z) \in D$, we conclude that $g(t, z, x) \neq 0$ for all $x \in S_{d}^{+}$. By applying the solutions of Cauchy's functional equation along rays $s \mapsto s x$ (see e.g. [1]) we conclude that for every $(t, z) \in D$ there exists a matrix $\psi(t, z) \in S_{d}+\mathrm{i} S_{d}$ such that

$$
g(t, z, x)=e^{-\langle\psi(t, z), x\rangle}
$$

holds for all $x \in S_{d}^{+}$. Moreover, we claim that $\Re(\psi(t, z)) \in S_{d}^{++}$. Indeed, we argue by contradiction and assume that $\Re(\psi(t, z)) \notin S_{d}^{++}$. Then, by self-duality of $S_{d}^{+}$, there exists some $x_{0} \in S_{d}^{+} \backslash\{0\}$ such that $\left\langle\Re(\psi(t, z)), r x_{0}\right\rangle \leq 0$ for all $r \geq 0$. Hence, $\left|g\left(t, z, r x_{0}\right)\right| \geq 1$, and thus

$$
0<|\Upsilon(t, z)| \leq\left|P_{t} e^{-\left\langle z, r x_{0}\right\rangle}\right| \leq P_{t} e^{-\left\langle\Re z, r x_{0}\right\rangle}=e^{-\phi(t, \Re z)-\left\langle\psi(t, \Re z), r x_{0}\right\rangle},
$$

by the real affine property of $X$. As Proposition 5.3 implies $\psi(t, \Re z) \in S_{d}^{++}$, it follows that the right hand side converges to 0 for $r \rightarrow \infty$, a contradiction.

It remains to show that $D=\mathbb{R}_{+} \times\left(S_{d}^{++}+\mathrm{i} S_{d}\right)$. Thereto fix $z \in S_{d}^{++}+\mathrm{i} S_{d}$ and define the discrete set of zeros $N=\{t \mid \Upsilon(t, z)=0\}$ of the analytic function $t \mapsto \Upsilon(t, z)$. We claim that $N=\emptyset$. Indeed, for every $t \notin N$ there exists some $s_{0}>0$ such that $t+s \notin N$ for all $s \in\left[0, s_{0}\right]$. Hence, by the Markov property of $X$ and (6.2), we infer

$$
\Upsilon(t+s, z)=\Upsilon(t, z) \Upsilon(s, \psi(t, z))
$$

for all $s \in\left[0, s_{0}\right]$. Since $|\Upsilon| \leq 1$ on $\mathbb{R}_{+} \times\left(S_{d}^{++}+\mathrm{i} S_{d}\right)$ and $N$ is discrete, we conclude from (6.3) that $t \mapsto|\Upsilon(t, z)|$ is a non-increasing function on $\mathbb{R}_{+}$. Hence, for any $t_{0} \in N$, we would have $\Upsilon(t, z)=0$ for all $t \geq t_{0}$. But this contradicts the fact that $\Upsilon(t, z)$ is analytic in $t \in \mathbb{R}_{+}$with $\Upsilon(0, z)=1$. Whence $N=\emptyset$, as desired.

Finally, notice by Lemma 6.1 that $\phi(t, u+\mathrm{i} v)$ and $\psi(t, u+\mathrm{i} v)$ in (6.1) can be chosen to be analytic in $(t, u, v) \in \mathbb{R}_{+} \times S_{d}^{++} \times S_{d}$ and continuously extended to $\mathbb{R}_{+} \times\left(S_{d}^{+}+\mathrm{i} S_{d}\right)$.

It remains to be shown that $\phi(\cdot, u+\mathrm{i} v)$ and $\psi(\cdot, u+\mathrm{i} v)$ solve (2.14) and (2.15) for all $u+\mathrm{i} v \in S_{d}^{+}+\mathrm{i} S_{d}$. First, note that it follows by dominated convergence that $F$ and $R$ are analytic on $S_{d}^{++}+\mathrm{i} S_{d}$ and continuous on $S_{d}^{+}+\mathrm{i} S_{d}$ (see e.g. 17. Lemma A.2] or [47, Theorem 25.17]). Hence, by standard ODE results ([15, Theorem 10.4.5]), for any initial point $u+\mathrm{i} v \in S_{d}^{++}+\mathrm{i} S_{d}$ there exists a unique local $\left(S_{d}^{++}+\mathrm{i} S_{d}\right)$-valued solution $\Psi(\cdot, u+\mathrm{i} v)$ of (2.15). Moreover, the maximal domain $\mathcal{D}=\left\{(t, u+\mathrm{i} v) \in \mathbb{R}_{+} \times\left(S_{d}^{++}+\mathrm{i} S_{d}\right) \mid \Psi(t, u+\mathrm{i} v) \in S_{d}^{++}+\mathrm{i} S_{d}\right\}$ is open and connected in $\mathbb{R}_{+} \times\left(S_{d}^{++}+\mathrm{i} S_{d}\right)$ ([2], Theorems 7.6 and 8.3]), and $\Psi$ is analytic on $\mathcal{D}$ ([15, Theorem 10.8.2]). By uniqueness of the solution, we have $\Psi(t, u)=\psi(t, u)$ for all $(t, u) \in \mathcal{D} \cap\left(\mathbb{R}_{+} \times S_{d}^{++}\right)$, see Proposition 5.3. On the other hand, Lemma 6.2 states that there exists an $\left(S_{d}^{++}+\mathrm{i} S_{d}\right)$-valued analytic extension of $\psi$ to $\mathbb{R}_{+} \times\left(S_{d}^{++}+\mathrm{i} S_{d}\right)$. Since $\mathbb{R}_{+} \times S_{d}^{++}$is a set of uniqueness, we conclude that $\Psi=\psi$ on $\mathcal{D}$ and, by its maximality, thus $\mathcal{D}=\mathbb{R}_{+} \times\left(S_{d}^{++}+\mathrm{i} S_{d}\right)$. A similar argument shows that $\phi(\cdot, u+\mathrm{i} v)$ is the unique global and analytic solution of (2.14) for $u+\mathrm{i} v \in S_{d}^{++}+\mathrm{i} S_{d}$. By a limiting argument, using the continuity of $F$ and $R$ on $S_{d}^{+}+i S_{d}$, it follows that $\phi(\cdot, u+\mathrm{i} v)$ and $\psi(\cdot, u+\mathrm{i} v)$ solve (2.14) and (2.15) also for $u+\mathrm{i} v \in S_{d}^{+}+i S_{d}$. Whence Theorem 2.5 is proved. 
6.3. Proof of Theorem 2.7, Let $X$ be a conservative affine process. It is shown in Proposition 4.11 that $\left\{e^{-\langle u, \cdot\rangle} \mid u \in S_{d}^{++}\right\} \subset D(\mathcal{A})$. Hence

$$
e^{-\left\langle u, X_{t}\right\rangle}-e^{-\langle u, x\rangle}-\int_{0}^{t} \mathcal{A} e^{-\left\langle u, X_{s}\right\rangle} d s
$$

is a $\left(\widetilde{\mathcal{F}}_{t}, \mathbb{P}_{x}\right)$-martingale with $\widetilde{\mathcal{F}}_{t}$ defined in (2.18). From [32, Theorem II.2.42], combined with (4.16) and Remark 2.6] it then follows that $X$ is a semimartingale with characteristics (2.19)-2.21). The canonical semimartingale representation (32, Theorem II.2.34]) of $X$ is thus given by

$$
\begin{aligned}
X_{t}= & x+B_{t}+X_{t}^{c}+\int_{0}^{t} \int_{S_{d}^{+} \backslash\{0\}} \chi(\xi)\left(\mu^{X}(d t, d \xi)-\nu(d t, d \xi)\right) \\
& +\int_{0}^{t} \int_{S_{d}^{+} \backslash\{0\}}(\xi-\chi(\xi)) \mu^{X}(d t, d \xi),
\end{aligned}
$$

where $X^{c}$ denotes the continuous martingale part, and $\mu^{X}$ the random measure associated to the jumps, of $X$. In order to establish representation (2.22), we find it convenient to consider the vectorization, $\operatorname{vec}\left(X^{c}\right) \in \mathbb{R}^{d^{2}}$, of $X^{c}$. The aim is now to find a $d^{2}$-dimensional Brownian motion $\widetilde{W}$ on a possibly enlarged probability space and a $d^{2} \times d^{2}$-matrix-valued function $\sigma$ such that

$$
\operatorname{vec}\left(X_{t}^{c}\right)=\int_{0}^{t} \sigma\left(X_{s}\right) d \widetilde{W}_{s} .
$$

Thus, $\sigma$ has to fulfill

$$
d\left\langle X_{i j}^{c}, X_{k l}^{c}\right\rangle_{t}=X_{t, i k} \alpha_{j l}+X_{t, i l} \alpha_{j k}+X_{t, j k} \alpha_{i l}+X_{t, j l} \alpha_{i k}=\left(\sigma\left(X_{t}\right) \sigma^{\top}\left(X_{t}\right)\right)_{i j k l} .
$$

As suggested by (5.6), we define the entries of the $d^{2} \times d^{2}$-matrix $\sigma(x)$ in terms of $\sigma^{k l}(x)$ given in (5.6) by

$$
\sigma_{i j k l}(x)=\sigma_{i j}^{k l}(x)=\sqrt{x}_{i k} \Sigma_{l j}+\Sigma_{i l}^{\top} \sqrt{x}_{j k} .
$$

Note that the $(k l)^{t h}$ column of $\sigma(x)$ is just the vectorization of the matrix $\sigma^{k l}(x)$. We thus obtain $A_{i j k l}(x)=\left(\sigma(x) \sigma^{\top}(x)\right)_{i j k l}$. Hence $\sigma(x)$ satisfies (6.5). Analogous to the proof of [45, Theorem 20.1], we can now build a $d^{2}$-dimensional Brownian motion $\widetilde{W}$ on an enlargement of the probability space such that (6.4) holds true. As the $(i j)^{t h}$ entry of $X^{c}$ is given by

$X_{t, i j}^{c}=\operatorname{vec}\left(X_{t}^{c}\right)_{i j}=\int_{0}^{t} \sum_{k, l=1}^{d} \sigma_{i j k l}\left(X_{s}\right) d \widetilde{W}_{s, k l}=\int_{0}^{t}\left(\sqrt{X_{s}} d W_{s} \Sigma+\Sigma^{\top} d W_{s}^{\top} \sqrt{X_{s}}\right)_{i j}$,

where $W$ is the $d \times d$-matrix Brownian motion satisfying $\operatorname{vec}(W)=\widetilde{W}$, we obtain the desired representation.

6.4. Proof of Theorem 2.10, We first prove some technical lemmas:

Lemma 6.3. Let $g: S_{d}^{+} \rightarrow \mathbb{R}$ be an additive function, that is, $g$ satisfies Cauchy's functional equation

$$
g(x+y)=g(x)+g(y), \quad x, y \in S_{d}^{+} .
$$


Then $g$ can be extended to an additive function $f: S_{d} \rightarrow \mathbb{R}$. Moreover, if $g$ is measurable on $S_{d}^{+}$then $f$ is measurable on $S_{d}$. In that case, $f$ is a continuous linear functional, that is, $f(x)=\langle c, x\rangle$ for some $c \in S_{d}$.

Proof. The first part follows from Lemma 4.4 .

Concerning measurability, let $E \in \mathcal{B}(\mathbb{R})$, a Borel measurable set. Then $f^{-1}(E)=$ $\bigcup_{n=1}^{\infty} B_{n}$, where $B_{n}:=f^{-1}(E) \cap B_{\leq n}(0)$. Hence, by the additivity of $f$ we have

$$
\begin{aligned}
f^{-1}(E) & =\bigcup_{n=1}^{\infty} B_{n}=\bigcup_{n=1}^{\infty}\left\{x+n I \mid x \in S_{d}, f(x) \in E,\|x\| \leq n\right\}-n I \\
& =\bigcup_{n=1}^{\infty}\left\{y \in S_{d} \mid f(y) \in E+f(n I),\|y-n I\| \leq n\right\}-n I \\
& =\bigcup_{n=1}^{\infty}\left\{y \in S_{d}^{+} \mid g(y) \in E+g(n I),\|y-n I\| \leq n\right\}-n I,
\end{aligned}
$$

which again is a measurable set, in view of the measurability of $g$ on $S_{d}^{+}$.

For $x \in S_{d}$ we write $x=\left(x_{i}\right)_{i}$ where $1 \leq i \leq \frac{d(d+1)}{2}$. We introduce the additive functions $f_{i}: \mathbb{R} \rightarrow \mathbb{R}$ via $f_{i}\left(x_{i}\right)=f\left(0, \ldots, 0, x_{i}, 0, \ldots, 0\right)$. By the just proved measurability of $f$ we infer that all $f_{i}$ are measurable functions on $\mathbb{R}$. By 1 , Chapter 2, Theorem 8], any additive measurable function on the real line is a continuous linear functional. Hence for each $i$ we infer the existence of $c_{i} \in \mathbb{R}$ such that $f_{i}\left(x_{i}\right)=c_{i} x_{i}$ holds. Since $f(x)=\sum_{i} f_{i}\left(x_{i}\right)$ it follows that $f(x)=\langle c, x\rangle$ for some $c \in S_{d}$.

Also, we consider Cauchy's exponential equation for $h: S_{d}^{+} \rightarrow \mathbb{R}_{+}$, that is,

$$
h(x+y)=h(x) h(y), \quad x, y \in S_{d} .
$$

Lemma 6.4. Suppose $h: S_{d}^{+} \rightarrow \mathbb{R}_{+}$is measurable, strictly positive, and satisfies (6.8). Then $h(x)=e^{-\langle c, x\rangle}$, for some $c \in S_{d}$. If $h \leq 1$, then $c \in S_{d}^{+}$.

Proof. Since $h$ is strictly positive, its logarithm yields the well defined function $g: S_{d}^{+} \rightarrow \mathbb{R}, \quad g(x):=\log h(x)$. Clearly $g$ is additive, hence by the first part of Lemma 6.3. there exists a unique additive extension $f: S_{d} \rightarrow \mathbb{R}$. Also, $f$ is measurable on $S_{d}^{+}$, hence by the second assertion of Lemma 6.3 we have $f(x)=$ $\langle c, x\rangle$, for some $c \in S_{d}$. The last statement follows from the monotonicity of the exponential and the self duality of $S_{d}^{+}$.

Remark 6.5. The assumption of strict positivity of $h$ in the preceding lemma is essential. Otherwise, there exist solutions $h$ which are not of the asserted form. Consider, for instance, $g(x):=1_{\partial S_{d}^{+}}$, which is a measurable multiplicative functional on $S_{d}^{+}$.

Lemma 6.4 is the main ingredient of the proof of the following characterization concerning $k$-fold convolutions of Markov processes:

Lemma 6.6. Let $\left(\mathbb{P}_{x}^{(i)}\right)_{x \in S_{d}^{+}} \in \mathcal{P}(i=0,1, \ldots, k)$. Then

$$
\mathbb{P}_{x_{1}}^{(1)} * \cdots * \mathbb{P}_{x_{k}}^{(k)}=\mathbb{P}_{x}^{(0)}, \quad \forall x_{i} \in S_{d}^{+}, \quad x=x_{1}+\cdots+x_{k},
$$

if and only if for all $\mathbf{t}=\left(t_{1}, \ldots, t_{N}\right) \in \mathbb{R}_{+}^{N}$ and $\mathbf{u}=\left(u^{(1)}, \ldots, u^{(N)}\right) \in\left(S_{d}^{+}\right)^{N}$, $N \in \mathbb{N}_{0}$, there exists $\rho^{(i)}(\mathbf{t}, \mathbf{u}) \in \mathbb{R}$ and $\psi(\mathbf{t}, \mathbf{u}) \in S_{d}$ such that $\prod_{i=1}^{k} \rho^{(i)}(\mathbf{t}, \mathbf{u})=$ 


$$
\begin{aligned}
& \rho^{(0)}(\mathbf{t}, \mathbf{u}) \text { and } \\
& \quad \mathbb{E}_{x}^{(j)}\left[e^{\sum_{i=1}^{N}\left\langle u^{(i)}, X_{t_{i}}\right\rangle}\right]=\rho^{(j)}(\mathbf{t}, \mathbf{u}) e^{-\langle\psi(\mathbf{t}, \mathbf{u}), x\rangle}, \quad \forall x \in S_{d}^{+}, \quad j=0,1, \ldots, k .
\end{aligned}
$$

Proof. We proceed similarly as in the proof of [17, Lemma 10.3]. Fix $k>1, N>1$, $\mathbf{t}, \mathbf{u}$ and set

$$
g^{(j)}(x):=\mathbb{E}_{x}^{(j)}\left[e^{\sum_{i=1}^{N}\left\langle u^{(i)}, X_{t_{i}}\right\rangle}\right] .
$$

By the very definition of convolution, (6.9) is equivalent to the following

$$
g^{(1)}\left(x_{1}\right) \cdots g^{(k)}\left(x_{k}\right)=g^{(0)}(x), \quad \forall x_{i} \in S_{d}^{+}, \quad x=x_{1}+\cdots+x_{k},
$$

Hence the implication (6.10) $\Rightarrow$ 6.11) is obvious. For the converse direction, we observe that $g^{(i)}$ are strictly positive on all of $S_{d}^{+}$, and, that by (6.11) we have

$$
g:=g^{(1)} / g^{(1)}(0)=\cdots=g^{(k)} / g^{(k)}(0)=g^{(0)} / g^{(0)}(0)
$$

and $g$ is a measurable, strictly positive function on $S_{d}^{+}$satisfying (6.8). Hence an application of Lemma 6.4 yields the validity of equation (6.10).

We are prepared to prove Theorem 2.10:

(i) $\Rightarrow$ (ii) Due to Lemma 6.6 infinite decomposability implies that $X$ is affine. Also, by the definition of infinite decomposability and by Lemma 6.6 we have that the $k$ th $\operatorname{root}\left(\mathbb{P}_{x}^{(k)}\right)$ for each $k \geq 1$ is an affine process with state space $S_{d}^{+}$with exponents $\psi(t, u)$ and $\phi(t, u) / k$. This implies that $\left(\mathbb{P}_{x}^{(k)}\right)_{x \in S_{d}^{+}}$has admissible parameters $\left(\alpha, b / k, \beta^{i j}, c / k, \gamma, m / k, \mu\right)$. Hence, the admissibility condition proved in Proposition 4.17 implies $b / k \succeq(d-1) \alpha \succeq 0$, for each $k$, which is impossible, unless $\alpha=0$.

(ii) $\Rightarrow$ (iii) follows from Proposition 5.12 in view of the Lévy-Khintchine form of $-\phi(t, \cdot)-\langle\psi(t, \cdot), x\rangle$, for each $t>0$.

(ii) $\Rightarrow$ (iii) By definition, every transition kernel $p_{t}(x, d \xi)$ of $X$ is infinitely divisible with Laplace transform $P_{t} f_{u}(x)=e^{-\phi(t, u)-\langle x, \psi(t, u)\rangle}$. For each $k \geq 2$, the maps $\phi^{(k)}:=\frac{\phi}{k}, \psi^{(k)}:=\psi$ satisfy the properties (3.1)-(3.2). Also, infinite divisibility implies that for each $t, x \in \mathbb{R}_{+} \times S_{d}^{+}$,

$$
Q_{t}^{(k)} f_{u}(x):=e^{-\phi^{(k)}(t, u)-\left\langle\psi^{(k)}(t, u), \frac{x}{k}\right\rangle}
$$

is the Laplace transform of a sub-stochastic measure on $S_{d}^{+}$. In conjunction with properties (3.1)-3.2) we may conclude that $Q_{t}^{(k)}$ gives rise to a Feller semigroup on $C_{0}\left(S_{d}^{+}\right)$, which is affine in $y=x / n$. Hence we have constructed for each $k \geq 1$, a $k$ th root of $X$, which is stochastic continuous, by the definition of its characteristic exponents $\phi^{(k)}, \psi^{(k)}$. Whence Theorem 2.10 is proved.

\section{Appendix A. Existence and Viability of a Class of Jump-Diffusions}

In this section, we study existence and viability in a non-empty closed convex set $D \subset \mathbb{R}^{n}$ of solutions to the equation

$$
X_{t}=x+\int_{0}^{t} b\left(X_{s}\right)+\int_{0}^{t} \sigma\left(X_{s}\right) d W_{s}+J_{t}
$$

where $b(x) \in C_{b}\left(\mathbb{R}^{n}, \mathbb{R}^{n}\right), \sigma(x) \in C_{b}\left(\mathbb{R}^{n}, \mathbb{R}^{n \times m}\right)$ are Lipschitz continuous maps, $W_{t}$ a standard $m$-dimensional standard Brownian motion and $J_{t}$ a finite activity jump 
process with state-dependent, absolutely continuous compensator $K\left(X_{t}, d \xi\right) d t$. We further assume that $x \mapsto K\left(x, \mathbb{R}^{n}\right)$ is bounded.

We tackle this problem in three steps. First, we derive some regularity and existence results for diffusion SDEs. These results are not in the standard literature, we thus provide full proofs. Second, we prove existence of a càdlàg solution $X$ for (A.1). Finally, we provide sufficient conditions for $X$ to be $D$-valued.

A.1. Diffusion Stochastic Differential Equations. Let $\left(\Omega, \mathcal{F},\left(\mathcal{F}_{t}\right), \mathbb{P}\right)$ be a filtered probability space satisfying the usual conditions and carrying an $m$-dimensional standard Brownian motion $W_{t}$. We consider the following diffusion SDE

$$
X_{t}=x+\int_{0}^{t} b\left(X_{s}\right) 1_{\{\theta \leq s\}} d s+\int_{0}^{t} \sigma\left(X_{s}\right) 1_{\{\theta \leq s\}} d W_{s},
$$

where $(\theta, x) \in[0, \infty] \times \mathbb{R}^{n}$ and $b$ and $\sigma$ are as above. Recall that $X$ is a solution of (A.2) if $X$ is continuous and (A.2) holds for all $t \geq 0$ a.s. In particular, note that this null set depends on $(\theta, x)$.

Lemma A.1. Fix $T>0$ and let $p \geq 2$. Furthermore, let $\Theta_{1}, \Theta_{2}$ be stopping times and for $i=1,2, U_{i}, \mathcal{F}_{\Theta_{i}}$-measurable random variables. Consider the following equations

$$
\begin{aligned}
& X_{t}=U_{1}+\int_{0}^{t} b\left(X_{s}\right) 1_{\left\{\Theta_{1} \leq s\right\}} d s+\int_{0}^{t} \sigma\left(X_{s}\right) 1_{\left\{\Theta_{1} \leq s\right\}} d W_{s} \\
& Y_{t}=U_{2}+\int_{0}^{t} b\left(Y_{s}\right) 1_{\left\{\Theta_{2} \leq s\right\}} d s+\int_{0}^{t} \sigma\left(Y_{s}\right) 1_{\left\{\Theta_{2} \leq s\right\}} d W_{s} .
\end{aligned}
$$

Then there exists a constant $C$ depending only on $p, T, n$, the Lipschitz constants of $b$ and $\sigma$ and $\|b\|_{\infty},\|\sigma\|_{\infty}$ such that for $0 \leq t \leq T$,

$$
\begin{aligned}
& \mathbb{E}\left[\sup _{s \leq t}\left\|X_{s}-Y_{s}\right\|^{p}\right] \\
& \quad \leq C \mathbb{E}\left[\left\|U_{1}-U_{2}\right\|^{p}+\left|\Theta_{1} \wedge T-\Theta_{2} \wedge T\right|^{\frac{p}{2}}+\int_{0}^{t} \sup _{u \leq s}\left\|X_{u}-Y_{u}\right\|^{p} d s\right] .
\end{aligned}
$$

Proof. By the same arguments as in the proof of [45, Lemma 11.5], we first obtain the following estimate

$$
\begin{aligned}
\sup _{s \leq t}\left\|X_{s}-Y_{s}\right\|^{p} \leq & 3^{p-1}\left(\left\|U_{1}-U_{2}\right\|^{p}+\left(\int_{0}^{t}\left\|b\left(X_{s}\right) 1_{\left\{\Theta_{1} \leq s\right\}}-b\left(Y_{s}\right) 1_{\left\{\Theta_{2} \leq s\right\}}\right\| d s\right)^{p}\right. \\
& \left.+\sup _{s \leq t}\left\|\int_{0}^{s}\left(\sigma\left(X_{u}\right) 1_{\left\{\Theta_{1} \leq u\right\}}-\sigma\left(Y_{u}\right) 1_{\left\{\Theta_{2} \leq u\right\}}\right) d W_{u}\right\|^{p}\right) .
\end{aligned}
$$


Moreover,

$$
\begin{aligned}
& \left(\int_{0}^{t}\left\|b\left(X_{s}\right) 1_{\left\{\Theta_{1} \leq s\right\}}-b\left(Y_{s}\right) 1_{\left\{\Theta_{2} \leq s\right\}}\right\| d s\right)^{p} \\
& \leq 2^{p-1}\left(\left(\int_{\left(\Theta_{1} \wedge \Theta_{2}\right) \wedge t}^{\left(\Theta_{1} \vee \Theta_{2}\right) \wedge t}\left\|b\left(X_{s}\right)\right\| d s\right)^{p}+\left(\int_{\left(\Theta_{1} \vee \Theta_{2}\right) \wedge t}^{t}\left\|b\left(X_{s}\right)-b\left(Y_{s}\right)\right\| d s\right)^{p}\right) \\
& \leq 2^{p-1}\left(K\left|\Theta_{1} \wedge t-\Theta_{2} \wedge t\right|^{p}+t^{p-1} \int_{0}^{t}\left\|b\left(X_{s}\right)-b\left(Y_{s}\right)\right\|^{p} d s\right) \\
& \leq K\left(t^{\frac{p}{2}}\left|\Theta_{1} \wedge t-\Theta_{2} \wedge t\right|^{\frac{p}{2}}+\int_{0}^{t} \sup _{u \leq s}\left\|X_{u}-Y_{u}\right\|^{p} d s\right) .
\end{aligned}
$$

For the stochastic integral part, we apply the Burkholder-Davis-Gundy inequality

$$
\begin{aligned}
& \mathbb{E}\left[\sup _{s \leq t}\left\|\int_{0}^{s}\left(\sigma\left(X_{u}\right) 1_{\left\{\Theta_{1} \leq u\right\}}-\sigma\left(Y_{u}\right) 1_{\left\{\Theta_{2} \leq u\right\}}\right) d W_{u}\right\|^{p}\right] \\
& \leq K \mathbb{E}\left[\left(\int_{0}^{t}\left\|\sigma\left(X_{u}\right) 1_{\left\{\Theta_{1} \leq u\right\}}-\sigma\left(Y_{u}\right) 1_{\left\{\Theta_{2} \leq u\right\}}\right\|^{2} d u\right)^{\frac{p}{2}}\right] \\
& \leq K \mathbb{E}\left[\left(\int_{\left(\Theta_{1} \wedge \Theta_{2}\right) \wedge t}^{\left(\Theta_{1} \vee \Theta_{2}\right) \wedge t}\left\|\sigma\left(X_{u}\right)\right\|^{2} d u\right)^{\frac{p}{2}}+\left(\int_{\left(\Theta_{1} \vee \Theta_{2}\right) \wedge t}^{t}\left\|\sigma\left(X_{u}\right)-\sigma\left(Y_{u}\right)\right\|^{2} d u\right)^{\frac{p}{2}}\right] \\
& \leq K \mathbb{E}\left[\left|\Theta_{1} \wedge t-\Theta_{2} \wedge t\right|^{\frac{p}{2}}+\int_{0}^{t}\left\|\sigma\left(X_{s}\right)-\sigma\left(Y_{s}\right)\right\|^{p} d s\right] \\
& \leq K \mathbb{E}\left[\left|\Theta_{1} \wedge t-\Theta_{2} \wedge t\right|^{\frac{p}{2}}+\int_{0}^{t} \sup _{u \leq s}\left\|X_{u}-Y_{u}\right\|^{p} d s\right],
\end{aligned}
$$

where $K$ always denotes a constant which varies from line to line. The last estimate in both inequalities follows from the the Lipschitz continuity of $b$ and $\sigma$. By assembling these pieces, the proof is complete.

Here is a fundamental existence result, which is not stated in this general form in the standard literature. Therefore we provide a full proof.

Theorem A.2. There exists a function $Z:[0, \infty] \times \mathbb{R}^{n} \times \Omega \times \mathbb{R}_{+} \rightarrow \mathbb{R}^{n}$ with the following properties:

(i) $Z(\theta, x, \omega, t)$ is continuous in $(t, \theta, x)$ for all $\omega$.

(ii) $Z$ is $\mathcal{B}\left([0, \infty] \times \mathbb{R}^{n}\right) \otimes \mathcal{P}$-measurable.

(iii) $Z(\theta, x, \omega, t)$ solves A.2 for all $(\theta, x)$.

(iv) Let $\Theta$ be a stopping time and $U$ an $\mathcal{F}_{\Theta}$ measurable random variable, then $X_{t}=Z(\Theta, U, t)$ solves

$$
X_{t}=U+\int_{0}^{t} b\left(X_{s}\right) 1_{\{\Theta \leq s\}} d s+\int_{0}^{t} \sigma\left(X_{s}\right) 1_{\{\Theta \leq s\}} d W_{s}
$$

Proof. For every $(\theta, x) \in[0, \infty] \times \mathbb{R}^{n}$, there exists a unique solution $X_{t}(\omega)=$ $\widetilde{Z}(\theta, x, \omega, t)$ of A.2), which is a consequence of the Lipschitz continuity of $x \mapsto$ $b(x) 1_{\{\theta \leq s\}}$ and $x \mapsto \sigma(x) 1_{\{\theta \leq s\}}$. Uniqueness is meant modulo indistinguishability. From estimate A.3 we can deduce for $x, y \in[-T, T]^{n}, 0 \leq \theta_{1}, \theta_{2} \leq T$ and 
$0 \leq t \leq T$

$$
\begin{aligned}
& \mathbb{E}\left[\sup _{s \leq t}\left\|\widetilde{Z}\left(\theta_{1}, x, t\right)-\widetilde{Z}\left(\theta_{2}, y, t\right)\right\|^{p}\right] \\
& \quad \leq K\left(\|x-y\|^{\frac{p}{2}}+\left|\theta_{1}-\theta_{2}\right|^{\frac{p}{2}}+\int_{0}^{t} \mathbb{E}\left[\sup _{u \leq s}\left\|\widetilde{Z}\left(\theta_{1}, x, t\right)-\widetilde{Z}\left(\theta_{2}, y, t\right)\right\|^{p}\right] d s\right) .
\end{aligned}
$$

for some constant $K$. Hence, by Gronwall's lemma

$$
\begin{aligned}
\mathbb{E}\left[\sup _{s \leq t}\left\|\widetilde{Z}\left(\theta_{1}, x, t\right)-\widetilde{Z}\left(\theta_{2}, y, t\right)\right\|^{p}\right] & \leq K e^{K T}\left(\|x-y\|^{\frac{p}{2}}+\left|\theta_{1}-\theta_{2}\right|^{\frac{p}{2}}\right) \\
& \leq C\left\|\left(\theta_{1}, x\right)-\left(\theta_{2}, y\right)\right\|^{\frac{p}{2}}
\end{aligned}
$$

Let now Dya $=\left\{j 2^{-k}, j \in \mathbb{Z}, k \in \mathbb{N}\right\}$ be the set of dyadic rational numbers in $\mathbb{R}$ and Dya $^{n}=$ Dya $\times \ldots \times$ Dya the set of dyadic rational numbers in $\mathbb{R}^{n}$. Furthermore, we define $M$ by $M=\operatorname{Dya}^{n+1} \cap\left([0, T] \times[-T, T]^{n}\right)$. By setting $p=2 n+4$, we can apply Kolmogorov's lemma. Indeed, analogous to the proof of [33, Theorem 2.8], we derive for all $\left(\theta_{1}, x\right),\left(\theta_{2}, y\right) \in M$ with $0<\left\|\left(\theta_{1}, x\right)-\left(\theta_{2}, y\right)\right\|<h(\omega)$, where $h$ is a positive valued random variable, and for all $\omega \in \Omega_{T}^{*}$, where $\Omega_{T}^{*} \in \mathcal{F}$ is some set depending on $T$ with $\mathbb{P}\left(\Omega_{T}^{*}\right)=1$, the following estimate:

$$
\sup _{s \leq t}\left\|\widetilde{Z}\left(\theta_{1}, x, \omega, s\right)-\widetilde{Z}\left(\theta_{2}, y, \omega, s\right)\right\| \leq \delta\left\|\left(\theta_{1}, x\right)-\left(\theta_{2}, y\right)\right\|^{\gamma},
$$

where $\gamma \in\left(0, \frac{1}{p}\right)$ and $\delta$ some constant. Let us now define $Z$ : If $\omega \notin \Omega_{T}^{*}$, then $Z(\theta, x, \omega, t)=x$ for $0 \leq t \leq T$. For $\omega \in \Omega_{T}^{*}$ and $(\theta, x) \in M, Z(\theta, x, \omega, t)=$ $\widetilde{Z}(\theta, x, \omega, t)$ for $0 \leq t \leq T$. If $(\theta, x) \in M^{c}$, we choose a sequence $\left(\theta_{n}, x_{n}\right)_{n \in \mathbb{N}} \subseteq M$ such that $\left(\theta_{n}, x_{n}\right) \rightarrow(\theta, x)$. By estimate A.5. $\widetilde{Z}\left(\theta_{n}, x_{n}, \omega, t\right)$ is a Cauchy sequence converging with respect to $\sup _{s \leq t}\|\cdot\|$. We can therefore set $Z(\theta, x, \omega, t)=$ $\lim _{n \rightarrow \infty} \widetilde{Z}\left(\theta_{n}, x_{n}, \omega, t\right)$. As we have uniform convergence in $t$, the resulting process $Z$ is jointly continuous in $(\theta, x, t)$. Furthermore, for every $(\theta, x), Z$ is a indistinguishable from $\widetilde{Z}$, that is

$$
\mathbb{P}[Z(\theta, x, t)=\widetilde{Z}(\theta, x, t) \text { for all } 0 \leq t \leq T]=1 .
$$

Indeed, for $(\theta, x) \in M$, this is clear and for $(\theta, x) \in M^{c}$, we have

$$
\mathbb{P}\left[\sup _{s \leq t}\left\|\widetilde{Z}\left(\theta_{n}, x_{n}, s\right)-\widetilde{Z}(\theta, x, s)\right\| \geq \varepsilon\right] \leq C \varepsilon^{-p}\left\|\left(\theta_{n}, x_{n}\right)-(\theta, x)\right\|^{\frac{p}{2}},
$$

which implies that $\widetilde{Z}\left(\theta_{n}, x_{n}, t\right) \rightarrow \widetilde{Z}(\theta, x, t)$ in probability. As $\widetilde{Z}\left(\theta_{n}, x_{n}, t\right) \rightarrow$ $Z(\theta, x, t)$ a.s., and thus in particular in probability, it follows that $Z(\theta, x, t)=$ $\widetilde{Z}(\theta, x, t)$ a.s. for all $0 \leq t \leq T$. Letting $T \rightarrow \infty$, then proves assertion(i).

Statement (ii) is then a consequence of (i) and the $\mathcal{F}_{t}$-measurability of $\omega \mapsto$ $Z(\theta, x, t, \omega)$, which is satisfied since $\mathcal{F}_{0}$ contains all null sets of $\mathcal{F}$.

Furthermore, property A.6 implies that $Z(\theta, x, t)$ is a solution of A.2 for all $(\theta, x)$, which yields assertion (iii)

In order to prove (iv) we proceed in two steps:

Step 1. We first assume that $\Theta$ and $U$ take finitely many values $\theta_{1}, \ldots, \theta_{k} \in[0, \infty]$ and $x_{1}, \ldots, x_{l} \in \mathbb{R}^{n}$, respectively. Denote

$$
A_{j}=\left\{\Theta=\theta_{j}\right\}, \quad B_{h}=\left\{U=x_{h}\right\} .
$$


Then

$$
Z(\Theta, U, t)=\sum_{j, h} 1_{A_{j} \cap B_{h}} Z\left(\theta_{j}, x_{h}, t\right)
$$

does the job. Indeed, as $A_{j} \cap B_{h}$ are disjoint and $A_{j} \cap B_{h} \in \mathcal{F}_{\theta_{j}}$ for all $j, h$, we have (see e.g. [37, page 39])

$$
\begin{aligned}
U & +\int_{0}^{t} b(Z(\Theta, U, s)) 1_{\{\Theta \leq s\}} d s+\int_{0}^{t} \sigma(Z(\Theta, U, s)) 1_{\{\Theta \leq s\}} d W_{s} \\
& =U+\int_{0}^{t} \sum_{j, h} 1_{A_{j} \cap B_{h}} b\left(Z\left(\theta_{j}, x_{h}, s\right)\right) 1_{\left\{\theta_{j} \leq s\right\}} d s \\
& +\int_{0}^{t} \sum_{j, h} 1_{A_{j} \cap B_{h}} \sigma\left(Z\left(\theta_{j}, x_{h}, s\right)\right) 1_{\left\{\theta_{j} \leq s\right\}} d W_{s} \\
& =\sum_{j, h} 1_{A_{j} \cap B_{h}}\left(x_{h}+\int_{0}^{t} b\left(Z\left(\theta_{j}, x_{h}, s\right)\right) 1_{\left\{\theta_{j} \leq s\right\}} d s\right. \\
& \left.+\int_{0}^{t} \sigma\left(Z\left(\theta_{j}, x_{h}, s\right)\right) 1_{\left\{\theta_{j} \leq s\right\}} d W_{s}\right) \\
& =\sum_{j, h} 1_{A_{j} \cap B_{h}} Z\left(\theta_{j}, x_{h}, t\right)=Z(\Theta, U, t)
\end{aligned}
$$

for all $t \geq 0$ a.s.

Step 2. For general $\Theta, U$, approximate $\Theta^{(k)} \downarrow \Theta$ by the simple stopping times

$$
\Theta^{(k)}= \begin{cases}j 2^{-k}, & (j-1) 2^{-k} \leq \Theta<j 2^{-k}, \quad j=1, \ldots, k 2^{k} \\ \infty, & k \leq \Theta .\end{cases}
$$

Let $U^{(l)}$ be a sequence of $\mathcal{F}_{\Theta}$-measurable random variables, each $U^{(l)}$ taking finitely many values, and $U^{(l)} \rightarrow U$ in $L^{2}$ (such $U^{(l)}$ obviously exists). Moreover, $\left\{\Theta^{(k)}=\right.$ $\left.\theta_{j}\right\} \cap\left\{U^{(l)}=x_{h}\right\} \in \mathcal{F}_{\theta_{j}}$ for all $j, h$ (see [33, Chapter 1, Problem 2.24]).

By Step 1, each $Z\left(\Theta^{(k)}, U^{(l)}\right)$ satisfies the respective SDE. Moreover, from estimate A.3 and Grownwall's lemma we deduce that for any $T>0$, there exists a constant $C$ such that

$$
\begin{aligned}
& \mathbb{E}\left[\sup _{t \leq T} \| Z\left(\Theta^{(k)}, U^{(l)}, t\right)\right.\left.-Z\left(\Theta^{\left(k^{\prime}\right)}, U^{\left(l^{\prime}\right)}, t\right) \|^{2}\right] \\
& \leq C e^{C T} \mathbb{E}\left[\left\|U^{(k)}-U^{\left(k^{\prime}\right)}\right\|^{2}+\left|\Theta^{(k)} \wedge T-\Theta^{\left(k^{\prime}\right)} \wedge T\right|\right] .
\end{aligned}
$$

Hence $Z\left(\Theta^{(k)}, U^{(l)}\right)$ is a Cauchy sequence and thus converging with respect to $\mathbb{E}\left[\sup _{t \leq T}\|\cdot\|^{2}\right]$, for all $T>0$, to some continuous process $X$ satisfying (A.4. On the other hand, by the continuity of $(\theta, x) \mapsto Z(\theta, x, t)$, we know that

$$
Z\left(\Theta^{(k)}, U^{(l)}, t\right) \rightarrow Z(\Theta, U, t)
$$

for all $\omega$ and $t \geq 0$. Again, by continuity of $t \mapsto Z(\Theta, U, t)$, we conclude that $Z(\Theta, U)=X$ up to indistinguishability, which proves the claim. 
A.2. Existence of Jump-diffusions. We now provide a constructive proof for the existence of a solution of (A.1) on a specific stochastic basis which is defined as follows.

- $\left(\Omega, \mathcal{F},\left(\mathcal{F}_{t}\right)_{t \geq 0}\right)$ is a filtered space, where $\Omega:=\Omega_{1} \times \Omega_{2}, \mathcal{F}_{t}:=\mathcal{G}_{t} \otimes \mathcal{H}_{t}$ and $\mathcal{F}=\mathcal{G} \otimes \mathcal{H}$ are precisely defined below. Note that we do not have a measure on $(\Omega, \mathcal{F})$ for the moment. The generic sample element will be denoted by $\omega=\left(\omega_{1}, \omega_{2}\right) \in \Omega$.

- $\left(\Omega_{1}, \mathcal{G},\left(\mathcal{G}_{t}\right)_{t \geq 0}, \mathbb{P}_{1}\right)$ is some filtered probability space satisfying the usual conditions and carrying an $m$-dimensional standard Brownian motion $W$. We shall consider the above diffusion SDE A.2) on $\Omega_{1}$ and thus obtain the respective solution $Z\left(\theta, x, \omega_{1}, t\right)$ satisfying the regularity properties of Theorem A.2

- $\left(\Omega_{2}, \mathcal{H}\right)$ is the canonical space for $\mathbb{R}^{n}$-valued marked point processes (see e.g. 31]): $\Omega_{2}$ consists of all càdlàg, piecewise constant functions $\omega_{2}$ : $\left[0, T_{\infty}\left(\omega_{2}\right)\right) \rightarrow \mathbb{R}^{n}$ with $\omega_{2}(0)=0$ and $T_{\infty}\left(\omega_{2}\right)=\lim _{n \rightarrow \infty} T_{n}\left(\omega_{2}\right) \leq \infty$, where $T_{n}\left(\omega_{2}\right)$, defined by $T_{0}=0$ and

$$
T_{n}\left(\omega_{2}\right):=\inf \left\{t>T_{n-1}\left(\omega_{2}\right) \mid \omega_{2}(t) \neq \omega_{2}(t-)\right\} \wedge \infty, \quad n \geq 1,
$$

are the successive jump times of $\omega_{2}$. We denote by

$$
J_{t}(\omega)=J_{t}\left(\omega_{2}\right)=\omega_{2}(t) \text { on }\left[0, T_{\infty}\left(\omega_{2}\right)\right)
$$

the canonical jump process, and let $\mathcal{H}_{t}=\sigma\left(J_{s} \mid s \leq t\right)$ be its natural filtration with $\mathcal{H}=\mathcal{H}_{\infty}$. Note that $T_{n}$ are $\left(\mathcal{H}_{t}\right)$ and $\left(\mathcal{F}_{t}\right)$-stopping times if interpreted as $T_{n}(\omega)=T_{n}\left(\omega_{2}\right)$.

The following statement is meant to be pointwise, referring to the filtered measure space $\left(\Omega, \mathcal{F},\left(\mathcal{F}_{t}\right)\right)$ without reference to a probability measure.

Lemma A.3. Let $Z\left(\theta, x, \omega_{1}, t\right)$ be as of Theorem A.Q. Then for an $\mathcal{F}_{T_{n}}$-measurable random variable $U\left(\omega_{1}, \omega_{2}\right)$ the process $Z\left(T_{n}\left(\omega_{2}\right), U\left(\omega_{1}, \omega_{2}\right), \omega_{1}, t\right)$ is

(i) continuous in $t$ for all $\left(\omega_{1}, \omega_{2}\right)$

(ii) $\mathcal{F}_{t}$-adapted on $\left\{T_{n} \leq t\right\}$.

Proof. The first assertion is a consequence of A.2 (ii). The second one follows from the $\mathcal{B}\left([0, \infty] \times \mathbb{R}^{n}\right) \otimes \mathcal{P}$-measurability of $Z\left(\theta, x, \omega_{1}, t\right)$, as stated in A.2 (iii), and the fact that $T_{n}$ and $U$ are $\mathcal{F}_{t}$-measurable on $\left\{T_{n} \leq t\right\}$.

Here is our existence result for A.1.

Theorem A.4. There exists a càdlàg $\mathcal{F}_{t}$-adapted process $X$ and a probability measure $\mathbb{P}$ on $(\Omega, \mathcal{F})$ with $\left.\mathbb{P}\right|_{\mathcal{G}}=\mathbb{P}_{1}$, such that $X$ is a solution of A.1) on $\left(\Omega, \mathcal{F},\left(\mathcal{F}_{t}\right), \mathbb{P}\right)$.

Proof. We follow the arguments in the proof of [22, Theorem 5.1], which is based on [31, Theorem 3.6], and proceed in three steps.

Step 1. We start by solving (A.1) along every path $\omega_{2}$. To this end, let us define recursively: $\Delta \omega_{2}(0)=\Delta \omega_{2}(\infty)=0, X_{0}^{(0)}=x$, and for $n \geq 1$ :

$$
X_{t}^{(n)}\left(\omega_{1}, \omega_{2}\right)= \begin{cases}Z\left(T_{n-1}\left(\omega_{2}\right), X_{T_{n-1}}^{(n-1)}\left(\omega_{1}, \omega_{2}\right)+\Delta \omega_{2}\left(T_{n-1}\right), \omega_{1}, t\right), & t \in[0, \infty), \\ x_{0}, & t=\infty,\end{cases}
$$

where $x_{0}$ is any fixed point in $D$ and $Z$ satisfies the properties of Theorem A.2 By induction, every $X^{(n)}$ is continuous in $t$ for all $\left(\omega_{1}, \omega_{2}\right)$ and by Lemma A.3, $\mathcal{F}_{t^{-}}$ adapted on $\left\{T_{n} \leq t\right\}$ since $X_{T_{n-1}}^{(n-1)}\left(\omega_{1}, \omega_{2}\right)+\Delta \omega_{2}\left(T_{n-1}\right)$ is $\mathcal{F}_{T_{n}}$-measurable. Thus, 
the process

$$
X_{t}\left(\omega_{1}, \omega_{2}\right)=\sum_{n \geq 1} X_{t}^{(n)}\left(\omega_{1}, \omega_{2}\right) 1_{\left\{T_{n-1} \leq t<T_{n}\right\}}
$$

is càdlàg $\mathcal{F}_{t^{-}}$-adapted and solves A.1) on $\left(\Omega_{1}, \mathcal{G},\left(\mathcal{G}_{t}\right), \mathbb{P}_{1}\right)$ for $t \in\left[0, T_{\infty}\left(\omega_{2}\right)\right)$ and any fixed path $\omega_{2}$.

Step 2. It remains to show that there exists a probability measure $\mathbb{P}$ such that $K\left(X_{t}, d \xi\right)$ is the compensator of $J$ and $\left.\mathbb{P}\right|_{\mathcal{G}}=\mathbb{P}_{1}$ holds true. For this purpose, we shall make use of [31, Theorem 3.6]. Let us define the following random measure $\nu$ by

$$
\nu(d t, d \xi)= \begin{cases}K\left(X_{t}, d \xi\right) d t, & t<T_{\infty} \\ 0, & t \geq T_{\infty}\end{cases}
$$

Observe that $\nu$ is predictable, since $X_{t}$ is càdlàg and $\mathcal{F}_{t}$-adapted. Theorem 3.6 in 31 now implies that there exists a unique probability kernel $\mathbb{P}_{2}$ from $\Omega_{1}$ to $\mathcal{H}$, such that $\nu$ is the compensator of the random measure $\mu$ associated to the jumps of $J$. On $(\Omega, \mathcal{F})$ we then define the probability measure $\mathbb{P}$ by $\mathbb{P}(d \omega)=$ $\mathbb{P}_{1}\left(d \omega_{1}\right) \mathbb{P}_{2}\left(\omega_{1}, d \omega_{2}\right)$ whose restriction to $\mathcal{G}$ is equal to $\mathbb{P}_{1}$.

Step 3. We finally show that $X$ defined by A.7 solves (A.1) on $\left(\Omega, \mathcal{F},\left(\mathcal{F}_{t}\right), \mathbb{P}\right)$ for all $t \geq 0$. Note that $W(\omega)=W\left(\omega_{1}\right)$ is an $\left(\Omega, \mathcal{F},\left(\mathcal{F}_{t}\right), \mathbb{P}\right)$-Brownian motion. This implies that $Z(\theta, x, \omega, t)=Z\left(\theta, x, \omega_{1}, t\right)$ is a solution of A.2 on $\left(\Omega, \mathcal{F},\left(\mathcal{F}_{t}\right), \mathbb{P}\right)$ satisfying the properties of Theorem A.2. It thus remains to show that $T_{\infty}=\infty$ $\mathbb{P}$-a.s. Let $\mu$ be the random measure associated to the jumps. As $x \mapsto K\left(x, \mathbb{R}^{n}\right)$ is bounded, we have for all $T \geq 0$,

$$
\mathbb{E}_{\mathbb{P}}\left[\mu\left([0, T] \times \mathbb{R}^{n}\right)\right]=\mathbb{E}_{\mathbb{P}}\left[\nu\left([0, T] \times \mathbb{R}^{n}\right)\right]=\mathbb{E}_{\mathbb{P}}\left[\int_{0}^{T} K\left(X_{t}, \mathbb{R}^{n}\right) d t\right] \leq C T
$$

for some constant $C$. This implies that $\mu\left([0, T] \times \mathbb{R}^{n}\right)<\infty$ a.s. for all $T \geq 0$ and hence $\mathbb{P}\left[T_{\infty}<\infty\right]=0$ or equivalently $T_{\infty}=\infty$ a.s.

A.3. Viability of Jump-diffusions. Consider a non-empty closed convex set $D \subset$ $\mathbb{R}^{n}$. We now provide sufficient conditions for the solution $X$ in (A.7) to be $D$-valued. This result is based on [14, Theorem 4.1]. We recall the notion of the normal cone

$$
N_{D}(x)=\left\{u \in \mathbb{R}^{n} \mid\langle u, y-x\rangle \geq 0, \text { for all } y \in D\right\}
$$

of $D$ at $x \in D$, consisting of inward pointing vectors. See e.g. 29, Definition III.5.2.3 ], except for a change of the sign.

Theorem A.5. Assume that $\sigma$ has also a Lipschitz continuous derivative. If

$$
\begin{aligned}
x+\operatorname{supp}(K(x, \cdot)) & \subseteq D \\
\left\langle\sigma^{i}(x), u\right\rangle & =0 \\
\left\langle b(x)-\frac{1}{2} \sum_{i=1}^{n} D \sigma^{i}(x) \sigma^{i}(x), u\right\rangle & \geq 0
\end{aligned}
$$

for all $u \in N_{D}(x)$ and $x \in D$, where $\sigma^{i}$ denotes the $i^{\text {th }}$ column of $\sigma$. Then, for every initial point $x \in D$, the process $X$ defined in (A.7D is a D-valued solution of (A.1). 
Proof. We have to show that $X_{t}=\sum_{n \geq 1} X_{t}^{(n)}\left(\omega_{1}, \omega_{2}\right) 1_{\left\{T_{n-1} \leq t<T_{n}\right\}} \in D$ a.s. for all $t \geq 0$. We proceed by induction on $n$. For $n=1, X_{t}^{(1)}$ is simply given by

$$
X_{t}^{(1)}=x+\int_{0}^{t} b\left(X_{s}^{(1)}\right) d s+\int_{0}^{t} \sigma\left(X_{s}^{(1)}\right) d W_{s} .
$$

Due to [14, Theorem 4.1], conditions A.10) and A.11 imply that for all $t \geq 0$, $X_{t}^{(1)} \in D$ a.s. Let us now assume that for all $t \geq 0, X_{t}^{(n-1)} \in D$ a.s., thus in particular $X_{T_{n-1}}^{(n-1)}=X_{T_{n-1}-} \in D$ a.s. If $T_{n-1}=\infty$, then we immediately obtain

$$
X_{t}^{(n)}=X_{T_{n-1}}^{(n-1)}+\Delta J_{T_{n-1}}=x_{0} \in D .
$$

Otherwise, let $f \in C_{b}\left(\mathbb{R}^{n}, \mathbb{R}_{+}\right)$satisfy $\operatorname{supp}(f) \subseteq D^{c}$. Then,

$$
\begin{aligned}
\mathbb{E}\left[f\left(X_{T_{n-1}}^{(n-1)}+\Delta J_{T_{n-1}}\right)\right] & =\mathbb{E}\left[f\left(X_{T_{n-1}-}+\Delta J_{T_{n-1}}\right)\right] \\
& =\mathbb{E}\left[\int_{\mathbb{R}^{n} \backslash\{0\}} f\left(X_{T_{n-1}-}+\xi\right) K\left(X_{T_{n-1}-}, d \xi\right)\right]=0,
\end{aligned}
$$

since by (A.9),$X_{T_{n-1}-}+\operatorname{supp}\left(K\left(X_{T_{n-1}-}, \cdot\right)\right) \subseteq D$ a.s. and $f(D)=0$. Hence, $f\left(X_{T_{n-1}}^{(n-1)}+\Delta J_{T_{n-1}}\right)=0$ a.s., implying that $X_{T_{n-1}}^{(n-1)}+\Delta J_{T_{n-1}} \notin \operatorname{supp}(f)$ a.s. As this holds true for all $f \in C_{b}\left(\mathbb{R}^{n}, \mathbb{R}_{+}\right)$with $\operatorname{supp}(f) \subseteq D^{c}$, it follows that $X_{T_{n-1}}^{(n-1)}+$ $\Delta J_{T_{n-1}} \in D$ a.s. Thus, again by [14, Theorem 4.1] and conditions (A.10) and (A.11)

$$
X_{t}^{(n)}=X_{T_{n-1}}^{(n-1)}+\Delta J_{T_{n-1}}+\int_{0}^{t} b\left(X_{s}^{(n)}\right) 1_{\left\{T_{n-1} \leq s\right\}} d s+\int_{0}^{t} \sigma\left(X_{s}^{(n)}\right) 1_{\left\{T_{n-1} \leq s\right\}} d W_{s}
$$

a.s. takes values in $D$, which proves the induction hypothesis. The definition of $X$ then yields the assertion.

\section{Appendix B. An Approximation Lemma on the Cone of Positive Semidefinite Matrices}

In this section we deliver a differentiable variant of the Stone-Weierstrass theorem for $C^{\infty}$-functions on $S_{d}^{+}$. This approximation statement is essential for the description of the generator of an affine semigroup, as is elaborated in Section 4.2

We employ multi-index notation in the sequel. For $n \geq 1$ a multi-index is an element $\boldsymbol{\alpha}=\left(\alpha_{1}, \ldots, \alpha_{n}\right) \in \mathbb{N}_{0}^{n}$ having length $|\boldsymbol{\alpha}|:=\alpha_{1}+\cdots+\alpha_{n}$. The factorial is defined by $\boldsymbol{\alpha} !:=\prod_{i=1}^{n} \alpha_{i}$ !. The partial order $\leq$ is understood componentwise, and so are the elementary operations,+- . That is, $\boldsymbol{\alpha} \geq \boldsymbol{\beta}$ if and only if $\alpha_{i} \geq \beta_{i}$ for $i=1, \ldots, n$ and in that case - is well defined. Moreover, for $\boldsymbol{\alpha} \geq \boldsymbol{\beta}$, the multinomial coefficient is defined by

$$
\left(\begin{array}{c}
\boldsymbol{\alpha} \\
\boldsymbol{\beta}
\end{array}\right):=\frac{\alpha !}{(\boldsymbol{\alpha}-\boldsymbol{\beta}) ! \boldsymbol{\beta} !} .
$$

We define the monomial $x^{\alpha}:=\prod_{i=1}^{n} x_{i}^{\alpha_{i}}$, and the differential operator $\partial^{\alpha}:=$ $\frac{\partial^{|\alpha|}}{\partial_{x_{1}}^{\alpha} \ldots \partial_{x_{d}}^{\alpha_{n}}}$. Corresponding to a polynomial $P(x)=\sum_{|\boldsymbol{\alpha}| \leq k} a_{\boldsymbol{\alpha}} x^{\boldsymbol{\alpha}}$ we introduce the differential operator $P(\partial):=\sum_{|\boldsymbol{\alpha}| \leq k} a_{\boldsymbol{\alpha}} \frac{\partial^{|\alpha|}}{\partial x^{\alpha}}$. 
Let $\mathcal{S}=\mathcal{S}\left(S_{d}\right)$ denote the locally convex space of rapidly decreasing $C^{\infty}$ functions on $S_{d}$ (see [46, Chapter 7] 10 , and define the space of rapidly decreasing $C^{\infty}$-functions on $S_{d}^{+}$via restriction

$$
\mathcal{S}_{+}=\left\{f=\left.F\right|_{S_{d}^{+}}: F \in \mathcal{S}\right\}
$$

Equipped with the increasing family of semi-norms

$$
p_{k,+}(f):=\sup _{x \in S_{d}^{+},|\boldsymbol{\alpha}+\boldsymbol{\beta}| \leq k}\left|x^{\boldsymbol{\alpha}} \partial^{\boldsymbol{\beta}} f(x)\right|,
$$

$\mathcal{S}_{+}$becomes a locally convex vector space (see [46, Theorem 1.37]).

For technical reasons, we also introduce for $\varepsilon \geq 0$ the semi-norms

$$
p_{k, \varepsilon}(f):=\sup _{x \in S_{d}^{+}+B_{\leq \varepsilon}(0),|\boldsymbol{\alpha}+\boldsymbol{\beta}| \leq k}\left|x^{\boldsymbol{\alpha}} \partial^{\boldsymbol{\beta}} f(x)\right|
$$

on $C^{\infty}\left(S_{d}\right)$, where $B_{\leq r}(y)=\left\{z \in S_{d} \mid\|z-y\| \leq r\right\}$ denotes the closed ball with radius $r$ and center $y$. Note that $p_{k,+}=p_{k, 0}$. We first give an alternative description of $\mathcal{S}_{+}$.

Lemma B.1. We have

$$
\mathcal{S}_{+}=\left\{f=\left.G\right|_{S_{d}^{+}}: G \in C^{\infty}\left(S_{d}\right) \text { and } \exists \varepsilon>0 \text { such that } p_{k, \varepsilon}(G)<\infty \forall k \geq 0\right\} .
$$

Proof. The inclusion $\subseteq$ is trivial. Hence we prove $\supseteq$. So let $f=\left.G\right|_{S_{d}^{+}}$for some $G \in C^{\infty}\left(S_{d}\right)$ with $p_{k, \varepsilon}(G)<\infty$ for all $k \geq 0$ for some $\varepsilon>0$.

We choose a standard mollifier $\rho \in C_{c}^{\infty}\left(S_{d}\right)$ supported in $B_{\leq \varepsilon / 2}(0)$ and satisfying $\rho \geq 0, \int \rho=1$. For $\delta>0$ we introduce the neighborhoods $K_{\delta}:=S_{d}^{+}+B_{\leq \delta}(0)$ of $S_{d}^{+}$. The convolution $\varphi:=\rho * 1_{K_{\varepsilon / 2}} \in C^{\infty}\left(S_{d}\right)$ of the indicator function for $K_{\varepsilon / 2}$ with $\rho$ satisfies $\varphi=1$ on $S_{d}^{+}$and it vanishes outside $K_{\varepsilon}$. Furthermore, all derivatives of $\varphi$ are bounded, since

$$
\begin{aligned}
\left\|\partial^{\boldsymbol{\alpha}} \varphi(x)\right\|=\left\|\int_{K_{\varepsilon / 2}} \partial^{\boldsymbol{\alpha}} \rho(y-x) d y\right\| & \\
=\left\|\int_{K_{\varepsilon / 2}-x} \partial^{\boldsymbol{\alpha}} \rho(z) d z\right\| & \leq \int_{B_{\leq \varepsilon / 2}(0)}\left\|\partial^{\boldsymbol{\alpha}} \rho(z)\right\| d z<\infty,
\end{aligned}
$$

where the last estimate holds because supp $\rho \subseteq B_{\leq \varepsilon / 2}(0)$.

Now we set $F:=G \cdot \varphi$. By construction $F \in C^{\infty}\left(S_{d}\right),\left.F\right|_{S_{d}^{+}}=f$ and $F$ vanishes outside $K_{\varepsilon}$, because $\varphi$ does. What is left to show is that $F \in \mathcal{S}$. Since $F$ vanishes outside $K_{\varepsilon}$, it is sufficient to deliver all estimates of its derivatives on $K_{\varepsilon}$.

Let $\boldsymbol{\alpha}, \boldsymbol{\beta} \in \mathbb{N}_{0}^{d(d+1) / 2}$, then we have by the Leibniz rule,

$$
\begin{aligned}
x^{\boldsymbol{\alpha}} \partial^{\boldsymbol{\beta}} F(x) & =x^{\boldsymbol{\alpha}} \sum_{0 \leq \boldsymbol{\delta} \leq \boldsymbol{\beta}}\left(\begin{array}{l}
\boldsymbol{\beta} \\
\boldsymbol{\delta}
\end{array}\right)\left(\partial^{\boldsymbol{\beta}-\boldsymbol{\delta}} \varphi(x)\right)\left(\partial^{\boldsymbol{\delta}} G(x)\right) \\
& =\sum_{0 \leq \boldsymbol{\delta} \leq \boldsymbol{\beta}}\left(\begin{array}{l}
\boldsymbol{\beta} \\
\boldsymbol{\delta}
\end{array}\right)\left(\partial^{\boldsymbol{\beta}-\boldsymbol{\delta}} \varphi(x)\right)\left(x^{\boldsymbol{\alpha}} \partial^{\boldsymbol{\delta}} G(x)\right)
\end{aligned}
$$

\footnotetext{
${ }^{10}$ Strictly speaking, we identify $\mathcal{S}\left(S_{d}\right)=\mathcal{S}\left(\mathbb{R}^{d(d+1) / 2}\right)$ via the isomorphism between $S_{d}$ and the standard Euclidean space $\mathbb{R}^{d(d+1) / 2}$.
} 
By assumption $x^{\boldsymbol{\alpha}} \partial^{\boldsymbol{\delta}} G$ is bounded on $K_{\varepsilon}$, and $\left(\partial^{\boldsymbol{\beta}-\boldsymbol{\delta}} \varphi(x)\right)$ is bounded on all of $S_{d}$. Hence by by the last equation we have $\sup _{x \in S_{d},|\boldsymbol{\alpha}+\boldsymbol{\beta}| \leq k}\left|x^{\boldsymbol{\alpha}} \partial^{\boldsymbol{\beta}} F(x)\right|<\infty$, for all $k \in \mathbb{N}_{0}$ which by definition means $F \in \mathcal{S}$.

Lemma B.2. Let $u \in S_{d}^{++}$. Then for each $\varepsilon \geq 0$, and for all $k \geq 0$ we have $p_{k, \varepsilon}(\exp (-\langle u, \cdot\rangle))<\infty$. In particular, we have

$$
f_{u}=\left.\exp (-\langle u, \cdot\rangle)\right|_{S_{d}^{+}} \in \mathcal{S}_{+} .
$$

That is, $f_{u}=\left.F_{u}\right|_{S_{d}^{+}}$for some $F_{u} \in \mathcal{S}$.

Proof. Since $u \in S_{d}^{++}$, there exists a positive constant $c$ such that $\langle u, x\rangle \geq c\|x\|$, for all $x \in S_{d}^{+}$. Hence we obtain by a straightforward calculation, $p_{k,+}(\exp (-\langle u, \cdot\rangle))<$ $\infty$, for all $k \geq 0$.

Next, let $\varepsilon>0$, and write $x=y+z$, where $y \in S_{d}^{+}$and $z \in B_{\leq \varepsilon}(0)$ and pick multi-indices $\boldsymbol{\alpha}, \boldsymbol{\beta} \in \mathbb{N}_{0}^{d(d+1) / 2}$. Then we have by the binomial formula,

$$
\begin{aligned}
x^{\boldsymbol{\alpha}} \partial^{\boldsymbol{\beta}} e^{-\langle u, x\rangle} & =x^{\boldsymbol{\alpha}} \cdot(-1)^{|\boldsymbol{\beta}|} u^{\boldsymbol{\beta}} e^{-\langle u, x\rangle} \\
& =(y+z)^{\boldsymbol{\alpha}} \cdot(-1)^{|\boldsymbol{\beta}|} u^{\boldsymbol{\beta}} e^{-\langle u, y+z\rangle} \\
& =(-1)^{|\boldsymbol{\beta}|} u^{\boldsymbol{\beta}} \sum_{0 \leq \boldsymbol{\gamma} \leq \boldsymbol{\alpha}}\left(\begin{array}{c}
\boldsymbol{\alpha} \\
\boldsymbol{\gamma}
\end{array}\right)\left(y^{\boldsymbol{\alpha}} e^{-\langle u, y\rangle}\right)\left(z^{\boldsymbol{\alpha}-\boldsymbol{\gamma}} e^{-\langle u, z\rangle}\right) .
\end{aligned}
$$

Now that $z$ ranges in a compact set, and since $p_{k,+}(\exp (-\langle u, \cdot\rangle))<\infty$ we see that $x^{\boldsymbol{\alpha}} \partial^{\boldsymbol{\beta}} e^{-\langle u, x\rangle}$ must be bounded uniformly in $x \in S_{d}^{+}+B_{\leq \varepsilon}(0)$. Hence $p_{k, \varepsilon}(\exp (-\langle u, \cdot\rangle))<$ $\infty$, for all $k \geq 0$.

Finally, the assertion concerning $f_{u}$ is a consequence of the first assertion in conjunction with the statement of Lemma B.1.

We are now prepared to deliver the following density result for the $\mathbb{R}$-linear hull $\mathcal{M}$ of $\left\{f_{u}, u \in S_{d}^{++}\right\}$in $\mathcal{S}_{+}$:

Theorem B.3. $\mathcal{M}$ is dense in $\mathcal{S}_{+}$.

Proof. Denote by $\mathcal{S}^{\prime}=\mathcal{S}^{\prime}\left(S_{d}\right)$ and $\mathcal{S}_{+}^{\prime}$ the topological dual of $\mathcal{S}$ and $\mathcal{S}_{+}$, respectively. The former, $\mathcal{S}^{\prime}$, is known as the space of tempered distributions. The distributional action is denoted by $\langle$,$\rangle and \langle,\rangle_{+}$for $\mathcal{S}^{\prime}$ and $\mathcal{S}_{+}^{\prime}$, respectively.

Now suppose by contradiction, that $\mathcal{M}$ is not dense in $\mathcal{S}_{+}$. Then by [46, Theorem 3.5] there exists $T_{1} \in \mathcal{S}_{+}^{\prime} \backslash\{0\}$ such that $T_{1}=0$ on $\mathcal{M}$. Hence $\left\langle T_{1}, f_{u}\right\rangle_{+}=0$, for all $u \in S_{d}^{++}$. The restriction $\left.f \mapsto f\right|_{S_{d}^{+}}$yields a continuous linear embedding $\mathcal{S} \hookrightarrow \mathcal{S}_{+}$. Hence the restriction $T$ of $T_{1}$ to $\mathcal{S}$, given by

$$
\langle T, \varphi\rangle:=\left\langle T_{1},\left.\varphi\right|_{S_{d}^{+}}\right\rangle_{+}, \quad \varphi \in \mathcal{S}\left(S_{d}\right),
$$

yields an element of $\mathcal{S}^{\prime}$ with $\operatorname{supp}(T) \subseteq S_{d}^{+}$. Pick an $F_{u} \in \mathcal{S}$ according to Lemma B.2 By the very definition of $T$, we have $\left\langle T, F_{u}\right\rangle=\left\langle T_{1}, f_{u}\right\rangle_{+}=0$, for all $u \in S_{d}^{++}$. By the Bros-Epstein-Glaser Theorem (see [42, Theorem IX.15]) there exists a function $G \in C\left(S_{d}\right)$ with $\operatorname{supp}(G) \subseteq S_{d}^{+}$, polynomially bounded, (that is for suitable constants $C, N$ we have $|G(x)| \leq C(1+\|x\|)^{M}$, for all $\left.x \in S_{d}^{+}\right)$and a 
real polynomial $P(x)$ such that $P(\partial) G=T$ in $\mathcal{S}^{\prime}$. Hence we obtain for any $u \in S_{d}^{++}$

$$
\begin{aligned}
0=\left\langle T, F_{u}\right\rangle & =\left\langle P(\partial) G, F_{u}\right\rangle=\left\langle G, P(-\partial) f_{u}\right\rangle \\
& =\int_{S_{d}^{+}} G(x) P(-\partial) F_{u}(x) d x=P(u) \int_{S_{d}^{+}} G(x) \exp (-\langle u, x\rangle) d x .
\end{aligned}
$$

But the last factor is just the Laplace transform of $G$. This implies $G=0$, hence $T=0$, which in turn implies that $T_{1}$ vanishes on all of $\mathcal{S}_{+}$, a contradiction.

\section{REFERENCES}

[1] J. Aczél and J. Dhombres. Functional equations in several variables, volume 31 of Encyclopedia of Mathematics and its Applications. Cambridge University Press, Cambridge, 1989. With applications to mathematics, information theory and to the natural and social sciences.

[2] H. Amann. Ordinary differential equations, volume 13 of de Gruyter Studies in Mathematics. Walter de Gruyter \& Co., Berlin, 1990. An introduction to nonlinear analysis, Translated from the German by Gerhard Metzen.

[3] O. E. Barndorff-Nielsen and N. Shephard. Modeling by Lévy processes for financial econometrics. In Lévy processes, pages 283-318. Birkhäuser Boston, Boston, MA, 2001.

[4] O. E. Barndorff-Nielsen and R. Stelzer. Positive-definite matrix processes of finite variation. Probab. Math. Statist., 27(1):3-43, 2007.

[5] H. Bauer. Probability theory, volume 23 of de Gruyter Studies in Mathematics. Walter de Gruyter \& Co., Berlin, 1996. Translated from the fourth (1991) German edition by Robert B. Burckel and revised by the author.

[6] M.-F. Bru. Diffusions of perturbed principal component analysis. J. Multivariate Anal., 29(1):127-136, 1989.

[7] M.-F. Bru. Wishart processes. Journal of Theoretical Probability, 4(4):725-751, 1991.

[8] B. Buraschi, A. Cieslak, and F. Trojani. Correlation risk and the term structure of interest rates. Working paper, University St.Gallen, 2007.

[9] B. Buraschi, P. Porchia, and F. Trojani. Correlation risk and optimal portfolio choice. Working paper, University St.Gallen, 2006.

[10] J. Da Fonseca, M. Grasseli, and F. Ielpo. Hedging (co)variance risk with variance swaps. Working paper, University St.Gallen, 2006.

[11] J. Da Fonseca, M. Grasseli, and F. Ielpo. Estimating the Wishart affine stochastic correlation model using the empirical characteristic function. Working paper, 2008.

[12] J. Da Fonseca, M. Grasseli, and C. Tebaldi. Option pricing when correlations are stochastic: an analytical framework. Review of Derivatives Research, 10(2):151-180, 2007.

[13] J. Da Fonseca, M. Grasseli, and C. Tebaldi. A multifactor volatility Heston model. J. Quant. Finance, 8(6):591-604, 2008.

[14] G. Da Prato and H. Frankowska. Invariance of stochastic control systems with deterministic arguments. Journal of Differential Equations, 200(1):18-52, 2004.

[15] J. Dieudonné. Foundations of modern analysis. Academic Press, New York, 1969. Enlarged and corrected printing, Pure and Applied Mathematics, Vol. 10-I.

[16] C. Donati-Martin, Y. Doumerc, H. Matsumoto, and M. Yor. Some properties of the Wishart processes and a matrix extension of the Hartman-Watson laws. Publ. Res. Inst. Math. Sci., 40(4):1385-1412, 2004.

[17] D. Duffie, D. Filipović, and W. Schachermayer. Affine processes and applications in finance. Ann. Appl. Prob., 13:984-1053, 2003.

[18] S. N. Ethier and T. G. Kurtz. Markov processes. Wiley Series in Probability and Mathematical Statistics: Probability and Mathematical Statistics. John Wiley \& Sons Inc., New York, 1986. Characterization and convergence.

[19] D. Filipović. Time-inhomogeneous affine processes. Stochastic Process. Appl., 115(4):639-659, 2005.

[20] D. Filipović. Term-Structure Models: A Graduate Course. Springer, 2009.

[21] D. Filipović and E. Mayerhofer. Affine diffusion processes: Theory and applications. In $A d-$ vanced Financial Modelling, volume 8 of Radon Ser. Comput. Appl. Math. Walter de Gruyter, Berlin, 2009. 
[22] D. Filipović, L. Overbeck, and T. Schmidt. Dynamic CDO term structure modeling. forthcoming in Mathematical Finance, 2009.

[23] G. H. Golub and C. F. Van Loan. Matrix computations. Johns Hopkins Studies in the Mathematical Sciences. Johns Hopkins University Press, Baltimore, MD, third edition, 1996.

[24] C. Gourieroux, A. Montfort, and R. Sufana. International money and stock market contingent claims. Working paper, CREST, CEPREMAP and University of Toronto, 2005.

[25] C. Gourieroux and R. Sufana. Wishart quadratic term structure models. Working paper, CREST, CEPREMAP and University of Toronto, 2003.

[26] C. Gourieroux and R. Sufana. Derivative pricing with Wishart multivariate stochastic volatility: application to credit risk. Working paper, CREST, CEPREMAP and University of Toronto, 2004.

[27] M. Grasselli and C. Tebaldi. Solvable affine term structure models. Math. Finance, 18(1):135$153,2008$.

[28] S. Heston. A closed-form solution for options with stochastic volatility with appliactions to bond and currency options. Rev. of Financial Studies.

[29] J.-B. Hiriart-Urruty and C. Lemaréchal. Convex analysis and minimization algorithms. I, volume 305 of Grundlehren der Mathematischen Wissenschaften [Fundamental Principles of Mathematical Sciences]. Springer-Verlag, Berlin, 1993. Fundamentals.

[30] R. A. Horn and C. R. Johnson. Topics in matrix analysis. Cambridge University Press, Cambridge, 1991.

[31] J. Jacod. Multivariate point processes: predictable projection, Radon-Nikodým derivatives, representation of martingales. Z. Wahrscheinlichkeitstheorie und Verw. Gebiete, 31:235-253, $1974 / 75$.

[32] J. Jacod and A. N. Shiryaev. Limit theorems for stochastic processes, volume 288 of Fundamental Principles of Mathematical Sciences. Springer-Verlag, Berlin, second edition, 2003.

[33] I. Karatzas and S. E. Shreve. Brownian motion and stochastic calculus, volume 113 of Graduate Texts in Mathematics. Springer-Verlag, New York, second edition, 1991.

[34] T. Kato. Perturbation theory for linear operators. Classics in Mathematics. Springer-Verlag, Berlin, 1995. Reprint of the 1980 edition.

[35] M. Keller-Ressel. Affine processes - Theory and applications in mathematical Finance. PhD thesis Vienna University of Technology, 2009.

[36] M. Keller-Ressel, W. Schachermayer, and J. Teichmann. Affine processes are regular. Preprint, 2009.

[37] D. Lamberton and B. Lapeyre. Introduction to stochastic calculus applied to finance. Chapman \& Hall/CRC Financial Mathematics Series. Chapman \& Hall/CRC, Boca Raton, FL, second edition, 2008.

[38] S. Lang. Real and functional analysis, volume 142 of Graduate Texts in Mathematics. Springer-Verlag, New York, third edition, 1993.

[39] M. Leippold and F. Trojani. Asset pricing with matrix affine jump diffusions. Working paper, 2008.

[40] E. Mayerhofer and A. Smirnov. A characterization of conservative affine markov processes. VIF Working Paper No. 23, Vienna Institute of Finance, 2009.

[41] R. Narasimhan. Several complex variables. The University of Chicago Press, Chicago, Ill.London, 1971. Chicago Lectures in Mathematics.

[42] M. Reed and B. Simon. Methods of modern mathematical physics. II. Fourier analysis, selfadjointness. Academic Press [Harcourt Brace Jovanovich Publishers], New York, 1975.

[43] D. Revuz and M. Yor. Continuous martingales and Brownian motion, volume 293 of Grundlehren der Mathematischen Wissenschaften [Fundamental Principles of Mathematical Sciences]. Springer-Verlag, Berlin, 1991.

[44] R. T. Rockafellar. Convex analysis. Princeton Landmarks in Mathematics. Princeton University Press, Princeton, NJ, 1997. Reprint of the 1970 original, Princeton Paperbacks.

[45] L. C. G. Rogers and D. Williams. Diffusions, Markov processes, and martingales. Vol. 2. Cambridge Mathematical Library. Cambridge University Press, Cambridge, 2000. Itô calculus, Reprint of the second (1994) edition.

[46] W. Rudin. Functional analysis. International Series in Pure and Applied Mathematics. McGraw-Hill Inc., New York, second edition, 1991. 
[47] K. Sato. Lévy processes and infinitely divisible distributions, volume 68 of Cambridge Studies in Advanced Mathematics. Cambridge University Press, Cambridge, 1999. Translated from the 1990 Japanese original, Revised by the author.

[48] Z. Semadeni. Banach spaces of continuous functions. Vol. I. PWN-Polish Scientific Publishers, Warsaw, 1971. Monografie Matematyczne, Tom 55.

[49] A. V. Skorohod. Random processes with independent increments, volume 47 of Mathematics and its Applications (Soviet Series). Kluwer Academic Publishers Group, Dordrecht, 1991. Translated from the second Russian edition by P. V. Malyshev.

[50] D. W. Stroock. Diffusion processes associated with Lévy generators. Z. Wahrscheinlichkeitstheorie und Verw. Gebiete, 32(3):209-244, 1975.

[51] P. Volkmann. Über die Invarianz konvexer Mengen und Differentialungleichungen in einem normierten Raume. Math. Ann., 203:201-210, 1973.

ETH ZÜrich, Departement Mathematik, RÄmistrasse 101, 8092 Zürich, Switzerland, Vienna Institute of Finance, Heiligenstädter Strasse 46-48, 1190 Vienna, Austria

E-mail address: christa.cuchiero@math.ethz.ch, damir.filipovic@vif.ac.at,

eberhard.mayerhofer@vif.ac.at, josef.teichmann@math.ethz.ch 